\title{
An epitope-resurfaced virus-like particle can induce broad neutralizing antibody against four serotypes of dengue virus
}

\author{
Authors: Wen-Fan Shen ${ }^{1 \S}$, Jedhan Ucat Galula ${ }^{2 \S}$, Jyung-Hurng Liu ${ }^{3 \S}$, Mei-Ying Liao ${ }^{2 \S}$, \\ Chang-Hao Huang', Yu-Chun Wang ${ }^{2}$, Han-Chung Wu ${ }^{4}$, Jian-Jong Liang ${ }^{5}$, Yi-Ling Lin ${ }^{5}$, \\ Matthew T. Whitney ${ }^{6}$, Gwong-Jen J. Chang ${ }^{6}$, Sheng-Ren Chen ${ }^{8}$, Shang-Rung Wu ${ }^{7,8^{*}}$ Day- \\ Yu Chao ${ }^{2 \S *}$
}

\footnotetext{
Affiliations:

${ }^{1}$ Ph.D. Program in Microbial Genomics, National Chung-Hsing University, Taichung, Taiwan.

${ }^{2}$ Graduate Institute of Microbiology and Public Health, College of Veterinary Medicine, National Chung-Hsing University, Taichung, Taiwan.

${ }^{3}$ Institute of Genomics and Bioinformatics, College of Life Science, National Chung-Hsing University, Taichung, Taiwan.

${ }^{4}$ Institute of Cellular and Organismic Biology, Academia Sinica, Taipei, Taiwan

${ }^{5}$ Institute of Biomedical Sciences, Academia Sinica, Taipei, Taiwan

${ }^{6}$ Division of Vector-Borne Diseases, Centers for Disease Control and Prevention, Fort Collins, Colorado, USA.

${ }^{7}$ Institute of Basic Medical Sciences, College of Medicine, National Cheng Kung University, Tainan, Taiwan

${ }^{8}$ Institute of Oral Medicine, College of Medicine, National Cheng Kung University, Tainan, Taiwan
}

$\S$ These authors contributed equally to this work

Word counts: 287 words (Abstract), 7595 words (main text)

Keywords: dengue, virus-like particles, maturity, broad neutralizing, antibody

Running title: Epitope-resurfaced dengue VLP

\section{* Corresponding authors}

Day-Yu Chao, Ph.D. 
32 Graduate Institute of Microbiology and Public Health, College of Veterinary Medicine

33 National Chung-Hsing University

34 Taichung 402, Taiwan

35 TEL:886-4-22840694

36 FAX:886-4-22852186

37 EMAIL: dychao@nchu.edu.tw

38 Or

39 Shang-Rung Wu, Ph.D.

40 Institute of Oral Medicine, College of Medicine,

$41 \quad$ National Cheng Kung University,

42 Tainan 701, Taiwan

43 TEL:886-6-2353535

$44 \quad$ FAX:886-6-2359885

45

EMAIL: z10208056@email.ncku.edu.tw 


\section{Abstract}

47

Dengue fever is caused by four different serotypes of dengue virus (DENV) which is the leading cause of worldwide arboviral diseases in humans. Virus-like particles (VLPs) containing flavivirus $\mathrm{prM} / \mathrm{E}$ proteins have been demonstrated to be a potential vaccine candidate; however, the structure of dengue VLP is poorly understood. Herein we show for the first time that mD2VLP particles possess a $\mathrm{T}=1$ icosahedral symmetry with a groove located within the $\mathrm{E}$ protein dimers near the 2-fold vertices that exposed highly overlapping, cryptic neutralizing epitopes through cryo-electron microscopy reconstruction. Mice vaccinated with highly matured virus-like particles derived from DENV serotype 2 (mD2VLP) can generate higher crossreactive (CR) neutralization antibodies (NtAbs) and were protected against all 4 serotypes of DENV through clonal expansion supported by hybridoma and B-cell repertoire analysis. Our results revealed that a "epitope-resurfaced" mature-form dengue VLP has the potential to induce quaternary structure-recognizing broad CR NtAbs. 
4

\section{Introduction}

Dengue virus (DENV), a member of the family Flaviviridae, is a mosquito-borne pathogen with four distinct serotypes, DENV-1 to DENV-4(Lindenbach and Rice). It has been estimated that DENV infects about 390 million individuals globally each year, resulting in 96 million clinically apparent infections ranging from mild fever to the life-threatening dengue hemorrhagic fever (DHF) or dengue shock syndrome (DSS)(Bhatt et al., 2013). Although the chimeric yellow fever 17D-derived, tetravalent dengue vaccine (CYD-TDV) has recently been approved by the governments of a few DENV-circulating countries, the unexpected low vaccine efficacy in dengue-naïve children or children younger than 6 years old has limited the use of this vaccine in the 9-45 age group living in endemic countries(Guy and Jackson, 2016). Developing a new strategy by either improving the efficacy of CYD-TDV(Aguiar et al., 2016;Halstead, 2017; World Health Organization, 2017) or the second-generation vaccine candidates(Kirkpatrick et al., 2016) is essential to broaden the coverage for all vulnerable populations.

75

(6)

neutralizing antibodies (NtAbs)(Roehrig, 2003;Pierson et al., 2008). The ectodomain of the E protein contains three distinct domains, EDI, EDII, and EDIII, which are connected by flexible hinges to allow rearrangement of domains during virus assembly, maturation and infection(Modis et al., 2003). However, the immune response from humans who have recovered from primary DENV infections is dominated by cross-reactive (CR), non-NtAbs which recognize mainly pre-membrane (prM) or fusion loop of the E (FLE) protein(Dejnirattisai et al., 2010). During virus replication, newly synthesized DENVs are assembled as immature particles 
02

in the endoplasmic reticulum at neutral $\mathrm{pH}$, followed by translocation through the trans-Golgi network and low-pH secretory vesicles(Chambers et al., 1990). The pr portion of the prM protein, positioned to cover the fusion loop (FL) peptide at the distal end of each E protein, prevents premature fusion during the virion maturation process along the egress pathway from the infected cells(Li et al., 2008;Kostyuchenko et al., 2013). For DENV to become fully infectious, the pr molecule has to be removed by a cellular furin protease during the egress process(Rodenhuis-Zybert et al., 2010). However, furin cleavage in the low-pH secretory vesicles is thought to be inefficient; hence, DENV particles released from infected cells are heterogeneous populations with different degrees of cleavage and release of the pr portion of prM to form the mature membrane (M) protein(Pierson and Diamond, 2012). Immature DENV (imDENV) particles consist of uncleaved prM protein and partially immature particles (piDENV) containing both prM and $\mathrm{M}$ proteins, while fully mature DENV (mDENV) contains only M protein(Junjhon et al., 2010;Pierson and Diamond, 2012). Functional analyses have revealed that a completely immature flavivirus lacks the ability to infect cells unless in the presence of anti-prM antibodies through a mechanism called antibody-dependent enhancement (ADE) of infection(Dejnirattisai et al., 2010;Rodenhuis-Zybert et al., 2010;Rodenhuis-Zybert et al., 2011;da Silva Voorham et al., 2012). ADE plays an important role in dengue pathogenesis, and is potentially modulated by the antibody concentration and the degree of virion maturity (Nelson et al., 2008;Rodenhuis-Zybert et al., 2015). Virus-like particles (VLPs) containing flavivirus prM/E proteins have been demonstrated to be a potential vaccine candidate, since their ordered E structures are similar to those on the 
105 virion surface and also undergo low-pH-induced rearrangements and membrane fusion similar to

106 viral particles(Allison et al., 1995). Also, VLP vaccines present several advantages since they are

107 highly immunogenic, non-infectious, and accessible to quality control as well as increased

108 production capacity. However, we theorized that the process of VLP maturation might be similar

109 to that of dengue viral particles, with heterogeneous pr-containing structures. In this study, we

110 engineered and characterized the structure of mature DENV-2 VLPs through cryo-EM. The

111 immunological properties of mature VLPs were further compared with its immature counterparts.

112

113

114 
115

116

117

118

119

120

121

122

123

124

125

126

127

128

129

130

131

132

133

134

135

136

\section{Results}

As we theorized, the process of VLP maturation are similar to that of dengue viral particles so that the wild-type particles (wtD2VLP) produced from the cells are partially immature containing both $\mathrm{prM}$ and $\mathrm{M}$ proteins (Figure S1 in Supplementary Material). To overcome this problem, we engineered the DENV-2 VLPs as completely mature particles by manipulating the furin cleavage site at the junction of pr and $\mathrm{M}$ in a DENV-2 VLP-expressing plasmid(Chang et al., 2003). For comparison, we also generated immature VLP (imD2VLPs) by mutating the minimal furin cleavage motif REKR to REST(Li et al., 2008), which resulted in completely uncleaved prM as detected by western blot and ELISA (Figure 1). Generation of mature DENV-2 VLP (mD2VLP) was much more challenging. First, we performed multiple sequence alignments of the $\mathrm{pr} / \mathrm{M}$ junction from several flaviviruses, including the distancerelated cell fusion agent virus (CFAV), and analyzed their furin cleavage potential using the algorithm PiTou 2.0(Tian et al., 2012). DENV-3 pr/M junction had the lowest predicted Pitou score of 6.87, while WNV had the highest score of 15.4 (Figure 1A). Secondly, we determined the cleavage efficiency of D2VLP by replacing P1-8 at the pr/M junction site of DENV-2 with sequences from different flaviviruses. Although the Pitou prediction of the CFAV pr/M junction did not result in a high score for cleavage, replacement with P1-8 of CFAV resulted in the most efficient furin cleavage for D2VLP, with only $31 \%$ of prM remaining uncleaved as compared to the $100 \%$ uncleaved imD2VLP (Figure 1A). An additional mutation in the P3 residue from $\mathrm{E}$ to $\mathrm{S}$ of the CFAV P1-8 construct reduced the prM signaling to $13.7 \%$ of that of imD2VLP. We thus named this construct with the replacement of CFAV P1-8 plus the P3 E to S mutation as mD2VLP. The pr/M cleavage of this construct was increased to nearly 90\% (Figure 1B). Similar 
137 to wtD2VLP purified from plasmids transfected culture supernatants, the mD2VLP and

138 imD2VLP showed consistent conformational integrity as ascertained by similar equilibrium

139 banding profiles in 5-25\% sucrose density gradients, as well as comparable particle size

140 distributions as determined by negative staining electron microscopy, and complex glycosylation

141 on the E proteins (Figure S2 in Supplementary Material).

Previous study has shown molecular organization of recombinant VLPs from tick-borne

144 encephalitis virus (TBEV)(Ferlenghi et al., 2001). However, the cryo-EM results showed low

145 resolution due to particle size heterogeneity and no immunological data can be derived from such

146 structure. To better understand if the structural organization of mD2VLP is similar to that of

TBEV and its immunological characteristics, purified mD2VLPs were subjected to cryo-EM

148 analysis. The results demonstrated that the mD2VLPs had a variable size distribution (Figure S3

149 in Supplementary Material). The size variation made improving the resolution challenging,

150 however we were able to determine the overall morphology and $\mathrm{E}$ arrangement of mD2VLP

151 based on current achieved resolution. Using a dengue virus-specific Mab (MAb32-6)(Li et al.,

152 2012), immunogold labeling detected the mD2VLPs as spherical particles with a diameter of

$153 \sim 31 \mathrm{~nm}$ (Figure S4 in Supplementary Material), which was also the major population as shown

154 in Supplementary Figure S3B. Besides, the particles with diameter of $\sim 31 \mathrm{~nm}$ had more solid

155 features in 2D image analyses (Figure S3 in Supplementary Material) and were able to obtain

156 stable structure than other groups. Therefore, we continued and focused on this population for

157 further 3D reconstruction and image analyses. The results showed that mD2VLPs with the diameter of $\sim 31 \mathrm{~nm}$ at a resolution of $13.1 \AA$ had a hollow structure and smooth surface with 
protrusions around the 5-fold positions (Figure 2A, left, and Figure S5 in Supplementary Material). Fitting the atomic E and M surface proteins (PDB: 3J27) into the cryo-EM density map showed that the mD2VLPs have 60 copies of $\mathrm{E}$ packed as dimers in a $\mathrm{T}=1$ icosahedral surface lattice (Figure 2A, right). The apparent differences of structural features on the mD2VLPs compared to the mature native virion particles(Kostyuchenko et al., 2013;Zhang et al., 2013a) was a slimmer lipid bilayer and the rearrangements of the surface proteins. First, the central section through the viron and m2DVLP reconstructions showed that the bilayer of m2DVLP was relatively thinner than that of viron. The distance between the exterior leaflet and the interior leaflet of m2DVLP and virion was $12 \AA$ and $14 \AA$, respectively (Figure 2B). A similar $\mathrm{T}=1$ icosahedral symmetry of the E-protein arrangement and thinner lipid bilayer were also found in TBEV VLPs(Ferlenghi et al., 2001). Second, interpretation of the map showed that E dimer subunits moved apart from each other causing less density in the intra-dimeric interphase (Figure 3C). Because of this loose interaction, a groove located within the E-protein dimer near the icosahedral 2-fold vertices on mD2VLP was noted (Figure 3A, right, and Figure S6 in Supplementary Material). Third, the central section showed that there was a solid density in the $\mathrm{H}$-layer, which is composed of $\mathrm{H}$ helices of $\mathrm{E}$ and $\mathrm{M}$ protein stem regions, while the density in the T-layer, which contains transmembrane helices of $\mathrm{E}$ and $\mathrm{M}$ proteins, was weaker in the VLP (Figure 2B). Each E protein consists of an E ecto-domain and a stem region that connects the ecto-domain to its transmembrane region. The stem contains two $\alpha$-helices, one of which interacts with the viral lipid membrane and the other with the E ecto-domain. This looser interaction of $\mathrm{E}$ protein dimers was further stabilized by the $\mathrm{E}$ protein rearrangement and the closer interaction with one of the $\alpha$-helices, which resulted in the solid density beneath the ecto- 
181

182

183

184

185

186

187

188

189

190

191

192

193

194

195

196

197

198

199

200

201

202

domain as shown in Figure 2B. It is worth noting that the stem region of this mD2VLP was replaced into Japanese Encephalitis viral (JEV) sequence; it was previously suggested that this replacement increased the hydrophobicity for the interaction with the lipid membrane and enhanced the secretion of dengue VLP(Purdy and Chang, 2005).

Next, we speculated that the structure of mD2VLP with a groove located within the Eprotein dimer could affect the epitope presentation on the surface of the particles. First, we calculated the solvent accessibility of mD2VLP and compared the results with that of DENV-2 virion. This rearrangement of $\mathrm{E}$ protein under $\mathrm{T}=1$ symmetry on the VLP surface exposed more accessible epitopes (48.2\%; 191 amino acid with $\geq 30 \%$ solvent accessibility among 396 amino acids of the entire E protein) compared to DENV-2 virions (43.4\%; 172 amino acid), particularly located at fusion loop, aa 239, aa 251-262 of EDII which together formed the groove, and at A strand, cd loop and G strand of EDIII which surrounded a 5-fold axis (Figure 3A, left, Figure S7 in Supplementary Material). Footprint analysis (Figure 3B) showed that those cryptic residues on the E protein, which were previously buried inside of the native DENV virion and only interacted with the broad NtAbs while the virion "breathed"(Lok, 2016), were better exposed on the m2DVLP. Specifically, the epitopes interacting with MAb 1A1D-2, which binds to the virus at $37^{\circ} \mathrm{C}$ (Lok et al., 2008); MAb 2D22, which could block two-thirds of all dimers on the virus surface, depending on the strain(Fibriansah et al., 2015a); and the 'E-dimer-dependent epitope', which is recognized by broadly neutralizing MAbs EDE1 and 2(Rouvinski et al., 2015), were all well-exposed in VLPs without steric hindrance. Second, a panel of murine MAb, recognizing different domains of E protein and used previously for antigenic mapping(Crill et al., 2012), was 
subjected to binding-ELISA (Figure S8 in Supplementary Material). The results confirmed that most of the Mabs preferentially binds to mD2VLP, in particularly Mabs recognizing domain I/II (Figure 4).

To further determine the influence of prM cleavage on the immunogenicity of D2VLP, we immunized groups of 4-week-old BALB/c mice with the purified wtD2VLP, imD2VLP or mD2VLP (4 $\mu \mathrm{g} /$ mouse) twice at a 4-week interval. To better represent the neutralizing antibodies at the late convalescent phase, serum samples collected at 8 weeks after the boost were analyzed by antigen-specific ELISA for antibody response against homologous D2VLP antigens with different maturity profiles. In order to precisely quantify the amount of denguespecific antibodies, we used the same quantity of purified VLPs in the antigen-capture ELISA (Figure 5A). WtD2VLP and imD2VLP induced similar ELISA titers of DENV-2-specific IgG antibody against three VLP antigens. However, stronger reactivity against the mature antigen was noted with mD2VLP immunization (1:15,347 vs. 1:6,381 vs. 2,529 for mD2VLP, imD2VLP and wtD2VLP, respectively) (Figure 5B). We also analyzed the neutralizing ability of these sera against the four serotypes of DENV using a 50\% antigen focus-reduction micro neutralization test $\left(\mathrm{FR} \mu \mathrm{NT}_{50}\right)$. The mD2VLP immunization group induced a higher and broader neutralizing antibody response against all 4 serotypes of DENV $\left(\mathrm{FR} \mu \mathrm{NT}_{50}\right.$ for DENV-1=1:331, DENV$2=1: 597$, DENV-3=1:70 and DENV-4=1:141), as compared to the imD2VLP vaccinated group $\left(\mathrm{FRNT}_{50}\right.$ for DENV-1=1:100, DENV-2=1:207 DENV-3=1:64 and DENV-4=1:76) (Figure 5C). 
Since the amino acid sequence is identical except for the mutations at the furin cleavage site, the difference in antibody binding and neutralizing activity between mD2VLP and imD2VLP vaccinated mice sera could result from the difference in induction of CR anti-prM non-NtAbs or anti-E NtAbs recognizing structure-dependent epitopes. To address whether the higher neutralization activity induced by mD2VLP was partly due to the reduction of anti-prM antibodies that are known to be cross-reactive but have no neutralizing activity(Dejnirattisai et al., 2010), we performed epitope-blocking ELISAs by using an anti-prM-specific monoclonal antibody (MAb 2H2). MAb 2H2 blocked only $7.59 \%$ of the activity of anti-mD2VLP sera but blocked up to $35.26 \%$ of antibodies from the imD2VLP-vaccinated groups (Figure S9 in Supplementary Material). To avoid steric hindrance due to Mab 2H2 binding, we performed site234 directed mutagenesis on three amino acids of pr protein based on the following criteria: (1) the conservation of amino acids among all four serotypes; (2) residues interacting with MAb 2H2(Wang et al., 2013); (3) residues not interfering with prM and E interaction(Li et al., 2008). As shown in supplementary Figure S10, K26P was a key residue, which significantly decreased the binding of both MAb 2H2 and 155-49 (another cross-reactive murine Mab recognizing pr protein(Huang et al., 2006)). However, only the mutant $\Delta 2 \mathrm{H} 2$-imD2VLP with F1A, K21D and $240 \mathrm{~K} 26 \mathrm{P}$ amino acid triple mutations completely prevent the binding of both MAb $2 \mathrm{H} 2$ and 155-49 241 but not that of DENV-2, E-specific Nt-MAb 3H5 (Figure S11 in Supplementary Material). The 242 sera from immunized mice were tested for their binding ability to the wild-type and mutant$243 \Delta 2 \mathrm{H} 2 \mathrm{imD} 2 \mathrm{VLP}$. The differences in binding of imD2VLP and $\triangle 2 \mathrm{H} 2 \mathrm{imD} 2 \mathrm{VLP}$ was greater for 244 the anti-imD2VLP sera than the anti-mD2VLP sera (Figure 5D and E), suggesting that compared 245 to mD2VLP, imD2VLP was more likely to induce 2H2-like anti-prM antibodies. Thus, 
mD2VLP has the advantage of eliciting lower levels of anti-prM CR antibody and inducing antibodies with greater neutralizing activity.

The preservation of neutralizing epitopes on the surface of VLPs is critical for efficient production of broadly neutralizing antibody responses. Recent studies have suggested that human monoclonal antibodies (MAbs) reactive with all dengue serotypes can neutralize DENV in the low picomolar range. These MAbs have a preference to bind at the envelope dimer epitopes preserved on virion particles with a high degree of maturity(Dejnirattisai et al., 2015;Rouvinski et al., 2015). We next investigated if a broad neutralization response to mD2VLP immunization was due to the induction of antibodies with a preference to bind at the $\mathrm{E}$ dimer epitopes preserved on the surface of mature VLP(Dejnirattisai et al., 2015;Rouvinski et al., 2015). The splenocytes from mD2VLP-vaccinated mice were used to perform fusion and generate hybridomas. Among 2,836 hybridomas screened, two MAbs with high reactivities to VLP measured by ELISA and FR $\mu \mathrm{NT}_{50}$ were shown in Figure 6. As shown in Figure 6A, DM8-6 is a serotype-specific MAb reactive only with DENV-2; while MAb DM25-3 recognized all four serotypes of DENV. Next, we tested the neutralizing activity of these two MAbs against four serotypes of DENV. DM8-6 showed good neutralizing activity against DENV-2 at a concentration of $0.037 \mu \mathrm{g} / \mathrm{mL}$ in $\mathrm{FR} \mu \mathrm{NT}_{50}$ but poorly neutralized the other three serotypes. Consistent with the ELISA results, DM25-3 neutralized all four serotypes in $\mathrm{FR}_{\mu \mathrm{NT}} \mathrm{T}_{50}$ at 0.32 , $0.38,0.24$ and $0.58 \mu \mathrm{g} / \mathrm{mL}$ for DENV-1 to DENV-4, respectively (Figure 6B). 
To determine if MAb DM25-3 recognized quaternary structure-dependent epitopes

268

269

270

271 presented only on mature virion particles(Fibriansah et al., 2015a;Rouvinski et al., 2015), DM253 was tested for its binding activity to both mD2VLP and imD2VLP using antigen-capture ELISA. MAb DM25-3 recognized mD2VLP very well, but reacted poorly with imD2VLP (Figure 6C). Next, we performed site-directed mutagenesis on the fusion loop peptide in domain II and A-strand in domain III, both of which are important binding regions for CR group/complex neutralizing antibodies(de Alwis et al., 2011 ;Tsai et al., 2013) (Figure S12 in

Supplementary Material). The results suggested that amino acid E-101 was likely important in the binding site of DM25-3. Residue 101 on the fusion loop of E protein is conserved among all four serotypes of DENV and can only be exposed when virion particles undergo low-pH induced conformational change or during the "breathing" state(Zhang et al., 2013b;Zhang et al., 2015). Figure 3C shows that residue 101 is located at the touching point between EDII and EDIII of the dimeric molecule. Since residue 101 is a critical residue in stabilizing E-protein dimers on DENV virion, mutation on 101 would disrupt viral particle formation(Zhang et al., 2013a;Rouvinski et al., 2017). However, mD2VLP with a mutation of residue 101 can still form particles (Figure S13 in Supplementary Material), which further support our cryoEM model that the E:E protein interactions on VLP are loose. When the E:E protein interaction was looser, residue 101 at the groove within the dimeric molecule was more exposed (64\%) than on the native virion (23.8\%, PDB:3J27) or on the native virion during the $37^{\circ} \mathrm{C}$ "breathing" state (45.3\%, PDB:3ZKO). We also generated a recombinant virus of DENV-2 whose EDIII was exchanged for a consensus EDIII(Chen et al., 2013) and the recombinant virus PL046cEDIII was recovered from the transfected cell culture supernatants for use in ELISA and FR $\mu$ NT. 
Compared to the parental DENV-2 strain PL046, there was significant loss of binding of MAb DM25-3 and immune mouse sera to PL046cEDIII (Figure 6D), suggesting that DM25-3 could be an E-dimer inter-domain antibody whose binding footprint is sensitive to amino acid changes in the EDIII domain. By comparing $\mathrm{FR} \mu \mathrm{NT}_{50}$ tests using murine antisera on both PL046 and PL046cEDIII viruses, we found that anti-mD2VLP sera showed a greater difference in neutralizing activity against PL046 and PL046cEDIII than did anti-imD2VLP sera, indicating a greater conformational dependence for anti-mD2VLP-triggered neutralization (Figure 6E). into 96-well plates to better understand DENV-2 prM/E-specific B-cell repertoires (Figure 7A). By analyzing the amino acid sequences of the RT-PCR products of the variable regions of Ig heavy-chain $(\operatorname{IgH})$ and light-chain $(\operatorname{IgL})$ genes, the B-cell response from mD2VLP-immunized mice was more complex than the imD2VLP-immunized animals, particularly the gene loci of IgH (Figure 7B). The IgH and IgL genes from the DM25-3-producing hybridoma were also analyzed and found to contain IgHV1-22*01 and IgKV3-12*01 (Figure S14 in Supplementary Material), which were clonally expanded as shown in Figure 7B. 
310 2003;Hughes et al., 2012;Galula et al., 2014). The advantage of using sucking mice to test

311 vaccine efficacy is that the protection from dengue virus challenge can only come from maternal

312 antibodies generated by vaccinated female mice and passively transferred to the their babies. The

313 immunization and challenge schedule is illustrated in Figure 8A. As shown in Figure 8B,

314 suckling mice born from mothers vaccinated with mD2VLP were all protected from challenge

315 with all four serotypes of dengue virus.

316

317 


\section{Discussions}

How VLP immunogens mimic their viral counterparts structurally and how the neutralizing epitopes are preserved on the VLP surface are of significant interest in the development of good vaccine candidates. Recent studies suggest that potent human neutralizing

322 antibodies with broad reactivity across dengue serocomplex can be generated from dengue 323 patients, particularly after secondary infection(Tsai et al., 2013;Dejnirattisai et al., 2015; Rouvinski et al., 2015). Our unique findings here suggested that mD2VLP, with a 325 scaffolding of multiple E-protein dimers similar to that of virion, is capable of inducing such CR antibodies with broad neutralizing activity. This monovalent mature-form dengue VLP with "epitope re-surfaced" has the potential to be the "epitope-focused" antigens when combined with other live attenuated dengue vaccine to induce higher and broader neutralizing antibodies(Rouvinski et al., 2017;Rey et al., 2018).

Very few studies explored the immunogenicity of prM-reduced antigens and its capability in inducing broad NtAbs(Keelapang et al., 2013;Suphatrakul et al., 2015;Metz et al., 2017; Rouvinski et al., 2017). Similar design of prM-reduced D2VLP was used as immunogens

334 but was produced from mosquito cells and such VLP required adjuvants to boost 335 immunity(Suphatrakul et al., 2015). Other attempts using E-dimers without the co-expression of 336 prM cannot only display the quaternary structure epitopes but also reduce the exposure of FLE 337 epitopes(Metz et al., 218;Metz et al., 2017;Rouvinski et al., 2017). However, the 338 immunogenicity of such E-dimers with only two copies of E could be lower than VLP. The mD2VLP used in this study was produced from mammalian cells with the features similar to 
340 dengue virion, such as glycosylation pattern, particle distribution in gradient after high-speed

341 centrifugation, epitopes preserved on the surface by the mapping of monoclonal antibodies, and

342 mostly importantly, multiple copies of E structurally packed as dimers on the particle surface

343 lattice (Fig S2 and S8 in Supplementary Material). Compared to imD2VLP (35\%), only 7.6\% of

344 prM-recognizing antibodies were induced by mD2VLP through 2H2-blocking assay. Current

345 dengue vaccine candidates in the market such as CYD-TDV or others in clinical trial are either

346 prM-possessing forms or E-protein monomer which is lack of quaternary epitopes(Wichmann et

347 al., 2017). Our study suggested that mD2VLP has the potential to be a safer immunogen as the

348 second-generation dengue vaccine.

In addition, mD2VLP-vaccinated mice produce antisera predominantly composed of

351 about $48 \%$ of DM25-3-like antibodies (Figure S15 in Supplementary Material). The packing of E

352 dimers on VLPs does not just preserve quaternary structure epitopes on the surface lattice as

353 suggested previously (Metz et al., 218; Crill et al., 2009), but also provides a unique surface-

354 accessible structure with increased epitope accessibility. Usually these epitopes are cryptic in

355 mature virions maintained at $28^{\circ} \mathrm{C}$ and in a neutral-pH environment(Lok, 2016). By

356 superimposing the E-dimer-dependent quaternary epitopes(Lok et al., 2008; Fibriansah et al.,

357 2015a;Rouvinski et al., 2015) onto the structure of mD2VLP, the binding footprints of these

358 antibodies were highly overlapping and formed a "neutralization-sensitive hotspot" on mD2VLP

359 (Figure S16 in Supplementary Material). Thus, the underlying mechanisms of why mD2VLPs

360 are able to stimulate an elevated and broader immune response are based on the following: (1)

361 epitope accessibility exposed at the grooves within E-protein dimers, which govern the 
362 generation of neutralizing antibodies; (2) removal of decoy epitopes presented on prM-

363 containing structures found in imD2VLPs; and (3) inter-dimeric epitope accessibility due to the

364 5-fold and 3-fold openings of the E protein arrangement of $\mathrm{T}=1$. Other mechanisms cannot be

365 excluded such as reducing the production of FLE-like antibodies (ie. MAb E53), which have a

366 preference of binding to spikes on noninfectious, immature flavivirions by engaging the highly

367 conserved fusion loop that has limited solvent exposure of the epitope on mature virions(Cherrier

368 et al., 2009).

Although the current obstacle in developing the second generation dengue vaccine is that dengue vaccine-induced neutralizing antibodies failed to correlate with or predict vaccine mediated protection(Moodie et al., 2018; Yang et al., 2018). However, studies of vesicular

373 stomatitis virus demonstrated that in vivo protection depended on minimum serum antibody 374 concentration, regardless of immunoglobulin subclass, avidity or in vitro neutralization 375 activity(Bachmann et al., 1997). Therefore, it is crucial to understand the type of neutralizing antibodies induced by vaccination and establish the association between the level of such neutralizing antibodies and protection(Flipse and Smit, 2015;Henein et al., 2017;Katzelnick et al., 2017). Recent studies suggested that the most potent neutralizing antibodies came from those recognizing quaternary epitopes on the smooth surface of dengue virions(Fibriansah et al., 380 2015b;Rouvinski et al., 2015;Crowe, 2017), Regardless of the mechanisms involved in NtAb 381 generation and protection from viral challenges, we have found that a mature form of 382 monovalent VLP from dengue virus serotype 2 with "epitope re-surfaced" is efficient in inducing 383 elevated and broadly NtAbs. When combined delivery with other live attenuated vaccine, such as 
384 CYD-TDV or dengue tetravalent vaccine, there is potential to provide not just T-cell immunity,

385 but also higher broad NtAb response through "epitope-focusing" while exploring the next

386 generation dengue vaccine in humans(Crill et al., 2012;Flipse and Smit, 2015;Rey et al., 2018).

389 grooves within the E protein dimeric molecules on the surface of particles induced highly

390 protective, NtAbs against heterologous dengue viruses from all four serotypes. These

391 characteristics strongly suggested this VLP with novel structure can combine with other live

392 attenuated dengue vaccine as "immune-focused" antigens to induce higher and broader

393 neutralizing antibodies. The strategy here may also provide a new direction for the development

394 of other flavivirus vaccines, including Zika virus. 


\section{Materials and Methods}

398

399

400

401

402

403

404

405

406

407

408

409

410

411

412

413

414

415

416

Ethics statement

This study was carried out in compliance with the guidelines for the care and use of laboratory animals of the National Laboratory Animal Center, Taiwan. The animal use protocol has been reviewed and approved by the Institutional Animal Care and Use Committee (IACUC) of National Chung Hsing University (Approval Number: 101-58). All efforts were made to minimize suffering of mice.

Viruses, cells and antibodies

The strains of DENV serotypes 1-4 used were Hawaii (Genbank accession: KM204119.1), 16681 (Genbank accession: KU725663.1), H87 (Genbank accession: KU050695.1) and H241 (Genbank accession: AY947539.1), respectively. COS-1 (ATCC: CRL 1650), Vero (ATCC: CRL 1587) and C6/36 cells (ATCC: CRL 1660) were grown in Dulbecco's modified Eagle's medium (DMEM, Gibco, Life Technologies, Grand Island, NY) and were supplemented with $10 \%$ heat-inactivated fetal bovine serum (FBS, Hyclone, ThermoFisher, MA), 0.1mM nonessential amino acids (Gibco, Life Technologies, Grand Island, NY), 7.5\% $\mathrm{NaHCO}_{3}$, 100U/ml penicillin, and 100ug/ml streptomycin; 5\% FBS was used for Vero cells. Cells were maintained at $37^{\circ} \mathrm{C}$ with $5 \% \mathrm{CO}_{2}$, except for $\mathrm{C} 6 / 36$ cells, which were maintained at $28^{\circ} \mathrm{C}$ without $\mathrm{CO}_{2}$. Parental DENV-2 strain PL046, generated from an infectious clone, and the domain IIIswapped PL046 (PL046cEDIII), with domain III of DENV-2 replaced by consensus sequence as 
417 described previously (Liang et al., 2009; Chen et al., 2013), were provided by one of the co418 authors, Dr. Y-L Lin. reactive antibodies (4G2, 4A1B-9, 6B3B-3, 6B6C-1, 5H3) recognizing E-protein of all four major pathogenic flavivirus serocomplexes; complex cross-reactive antibodies (T5-1) recognizing all four DENV serocomplex viruses; serotype-specific MAb 3H5-1, recognizing DENV-2 only; rabbit sera and mouse hyper-immune ascitic fluid (MHIAF) for DENV-1 to 4 were provided by one of the authors, G-J Chang (DVBD, CDC, Fort Collins, CO). DENV-2 E protein-specific MAb 32-6 was provided by Dr. H-C Wu (Academia Sinica, TW), while DENV2 prM-specific MAb 155-49 was obtained from H-Y Lei (National Cheng Kung University, Taiwan). MAb $2 \mathrm{H} 2$ was also labeled with biotin using EZ-Link ${ }^{\mathrm{TM}}$ Sulfo-NHS-Biotin kit (Thermo Fisher Scientific Inc., Rockford, IL) according to the manufacturer's instructions. In order to increase the recognition of $\mathrm{M}$ protein, anti-M antibody was produced by cloning the complete $\mathrm{M}$ protein sequence into pET21a (Novagen, Germany) and then purifying the expressed protein under denaturing conditions as described in the molecular cloning handbook.

432 Ten micrograms $(10 \mu \mathrm{g})$ of purified M protein with Freud's complete adjuvant was used to 433 immunize the mice by the intraperitoneal route five times at 2 -week intervals. Anti-mouse 434 CD45R/B220 antibody conjugated with APC (allophycocyanin) and goat anti-mouse IgG conjugated with PE (phycoerythrin) were purchased from BioLegend (San Diego, CA).

Previously constructed and characterized recombinant plasmid pVD2, expressing the prM, $43780 \%$ and $20 \% \mathrm{COOH}$ terminus of the envelope proteins of DENV-2 (Asian 1 genotype, strain 438 16681) and Japanese encephalitis virus (strain SA14-14-2), respectively (Galula et al., 2014), 
439 was used in this study. The furin cleavage site of prM was mutated in pVD2 to generate a prM-

440 uncleaved plasmid or as indicated in Figure 1A by using site-directed mutagenesis following the

441 manufacturer's protocol (Stratagene, La Jolla, CA). The primers used for cloning and site-

442 directed mutagenesis are provided in Table S1. Nucleotide sequencing confirmed that all the

443 plasmids contained no other mutations other than those indicated.

Sequence analysis of flavivirus prM sites

Flavivirus prM protein amino acid sequence alignments were performed using ClustalX

2.1 software with the representative strains and the GenBank accession numbers as follows:

448 dengue virus serotype 2 (NP_056776), dengue virus serotype 1 (AIU47321), dengue virus serotype 3 (YP_001621843), dengue virus serotype 4 (NP_073286), Japanese encephalitis virus

450 (NP_775664), St. Louis encephalitis virus (AIW82235), West Nile virus (AIO10814), tick-borne

451 encephalitis virus (NP_775501), yellow fever virus (NP_041726), cell-fusion agent virus

452 (NP_041725), Zika virus (BAP47441.1). Sequences of prM junction region were scored for their

453 predicted cleavability by furin using the PiTou 2.0 software package(Tian et al., 2012). A

454 negative score indicates a sequence predicted not to be cleaved by furin, whereas a positive score

455 denotes prediction of furin cleavability.

Antigen production and purification

To produce virus-like particle (VLP) antigens, COS-1 cells at a density of $1.5 \times 10^{7}$ 
460 described protocol (Chang et al., 2000). After electroporation, cells were seeded into $75-\mathrm{cm}^{2}$ 461 culture flasks (Corning Inc., Corning, NY, USA) containing $15 \mathrm{~mL}$ growth medium and allowed 462 to recover overnight at $37^{\circ} \mathrm{C}$. The growth medium was replaced the next day with a maintenance 463 medium containing serum-free medium (SFM4MegaVirTM, SH30587.01, Hyclone, 464 ThermoFisher) supplemented with non-essential amino acid (NEAA), GlutaMAX, sodium 465 pyruvate and cholesterol (Gibco, Life Technologies, Grand Island, NY), and cells were 466 continuously incubated at $28^{\circ} \mathrm{C}$ with $5 \% \mathrm{CO}_{2}$ for VLP secretion. Tissue-culture media were 467 harvested 3 days after transfection and clarified by centrifugation at $8,000 \times \mathrm{g}$ for 30 minutes 468 (mins) at $4^{\circ} \mathrm{C}$ in AF-5004CA rotor (Kubota, Tokyo, Japan) using a Kubota 3740 centrifuge. The 469 harvested media were first concentrated 20-fold using 100K Amicon Ultra centrifugal filters 470 (Merck Millpore, CA) before loading onto a $20 \%$ sucrose cushion and concentrated by 471 ultracentrifugation at $28,000 \mathrm{rpm}$ for 16 hours at $4^{\circ} \mathrm{C}$ in a Beckman SW28 rotor. Purified VLPs 472 were resuspended in $250 \mu \mathrm{l}$ TNE buffer $(50 \mathrm{mM}$ Tris- $\mathrm{HCl}, 100 \mathrm{mM} \mathrm{NaCl}, 0.1 \mathrm{mM}$ EDTA, pH 7.4) 473 for every liter of harvested medium at $4{ }^{\circ} \mathrm{C}$ overnight. The VLPs were further purified by rate 474 zonal centrifugation in a 5 to $25 \%$ sucrose gradient at $25,000 \mathrm{rpm}$ at $4^{\circ} \mathrm{C}$ for 3 hours. All 475 gradients were made with TNE buffer and were centrifuged in a Beckman SW41 rotor. Fractions 476 of $0.5 \mathrm{ml}$ were collected by upward displacement and assayed by antigen-capture ELISA. For experiments that required highly purified VLPs, proteins with the peak OD values from antigen478 capture ELISA were pelleted at $40,000 \mathrm{rpm}$ at $4^{\circ} \mathrm{C}$ for 4 hours using a Beckman SW41 rotor, and 479 re-suspended in $250 \mu \mathrm{l}$ TNE buffer. The protein concentration was measured by the Bradford 480 assay (BioRad, Hercules, CA) following the commercial protocol and using bovine serum 481 albumin (BSA, New England Biolabs, MA) as a standard. Purified VLPs were also labeled with 
482 fluorescein for antigen-specific B-cell sorting following the manufacture's protocol(Zhang et al., 483 2010). (specific for DENV-2 domain III) and MAb 155-49 (specific for DENV prM). The ratio was (imD2VLP), which contained amino acid mutations at P1 and P2 sites from amino acid residue $\mathrm{R} / \mathrm{K}$ to $\mathrm{T} / \mathrm{S}$ (Li et al., 2008), respectively, was used as a standard to calculate the percentage of prM cleavage. Percent cleavage of prM was then calculated with reference to imD2VLP, which was assumed to be $100 \%$ uncleaved, as previously described (Dejnirattisai et al., 2015). and $50 \mu \mathrm{L}$ of each dilution was added to wells in duplicate, incubated for $2 \mathrm{hrs}$ at $37^{\circ} \mathrm{C}$, and 
detected by adding $50 \mu \mathrm{L}$ of anti-DENV-2 MHIAF at 1:2,000 in blocking buffer, incubated for 1 hr at $37^{\circ} \mathrm{C}$, and washed for five times. Fifty microliters of HRP-conjugated goat anti-mouse $\operatorname{IgG}$ (Jackson ImmunoResearch, Westgrove, PA, USA) at 1:5,000 in blocking buffer was added to wells and incubated for $1 \mathrm{hr}$ at $37^{\circ} \mathrm{C}$ to detect MHIAF. Subsequently, plates were washed ten times. Bound conjugate was detected with 3,3',5,5'-tetramethylbenzidine substrate (Enhanced KBlue $^{\circledR}$ TMB, NEOGEN ${ }^{\circledR}$ Corp., Lexington, KY, USA), after incubation at room temperature for 10 mins, and addition of $2 \mathrm{~N} \mathrm{H}_{2} \mathrm{SO}_{4}$ to stop the reaction. Reactions were measured at $\mathrm{A}_{450}$ using a Sunrise $^{\mathrm{TM}}$ TECAN microplate reader (Tecan, Grödig, Austria). The capability of antigencapture ELISA to detect imD2VLP or mD2VLP was measured against purified D2VLP at known total protein concentration from individual preparations. Data are expressed as P/N ratio by dividing the OD450 value from each dilution of mouse sera or MAb by the OD450 value from the control COS-1 culture supernatant.

vaccination mouse sera using the same antigen-capture ELISA protocol described above with minor modifications. Equal amounts of purified antigens were added into each well of an Agcapture ELISA plate. The concentration of purified antigens were determined from the standard curves generated using a sigmoidal dose-response analysis using GraphPad Prism (version 6.0, GraphPad Software, Inc., La Jolla, CA, USA). Individual mouse sera, collected from mice which had the same immunization schedule, initially diluted at 1:1,000, were titrated two-fold and added into wells in duplicate, and were incubated for $1 \mathrm{hr}$ at $37^{\circ} \mathrm{C}$. Pre-vaccination mouse sera were used as negative controls. Incubations with conjugate and substrate were carried out 
according to the standard Ag-capture ELISA as outlined. The $\mathrm{OD}_{450}$ values, modeled as nonlinear functions of the $\log 10$ serum dilutions using a sigmoidal dose-response (variable slope) equation and endpoint antibody titers from two independent experiments, were determined as the dilutions where the OD value was twice the average OD of a negative control.

Epitope-blocking ELISAs were performed to determine the vaccinated mouse response to the prM protein. The setup was similar to the IgG ELISA wherein plates were coated with rabbit anti-DENV-2 VLP serum, and blocked with 1\% BSA in PBS. After washing, pooled mouse sera were diluted two-fold in blocking buffer starting from 1:1,000, and were then incubated with imD2VLP antigen (pre-titrated to $\mathrm{OD}_{450}=1.0$ ) for $1 \mathrm{hr}$ at $37^{\circ} \mathrm{C}$. After serum incubation and washing, MAb $2 \mathrm{H} 2$ conjugated with biotin by EZ-Link Sulfo-NHS-Biotin (ThermoFisher, CA) at 1:4000 dilution was added to each well and incubated for $1 \mathrm{hr}$ at $37^{\circ} \mathrm{C}$ to compete with the already-bound antibody from the immune mouse sera specific for the imD2VLP antigen. Bound MAb 2H2 conjugate was detected with 1:1000 HRP-conjugated streptavidin (016-030-084, Jackson ImmunoResearch), and was incubated for $1 \mathrm{hr}$ at $37^{\circ} \mathrm{C}$. After washing with PBS for ten times, TMB substrate was added into the wells and the plates were incubated for 10 mins, and the reaction was stopped with $2 \mathrm{~N} \mathrm{H}_{2} \mathrm{SO}_{4}$. Reactions were measured at $\mathrm{A}_{450}$. Percent blocking was determined by comparing replicate wells with Biotin-conjugated MAb competing against pre-adsorbed naïve mouse serum using the formula: \% Blocking $=[\mathrm{OD} 450$ of imD2VLP-OD 450 of imD2VLP blocked by MAb 2H2)/OD 450 of imD2VLP]×100. 
Binding-ELISAs were used to assess the binding activity of MAbs or mouse immune sera to D2VLP or mutant antigens using a similar antigen-capture ELISA set-up, except that two-fold dilutions of the specific MAb or immune mouse sera replaced the anti-DENV-2 MHIAF. Equal

549 amounts of D2VLP antigens were added into wells, and were standardized using purified D2VLPs. The antibody endpoint reactivity was determined in a similar manner to the

551 determination of antigen endpoint secretion titers.

Equal amounts of purified VLPs and sample buffer were mixed and analyzed by $12 \%$

PAGE)(Schägger, 2006). For immunodetection, proteins were blotted from gels onto nitrocellulose membranes (iBlot ${ }^{\circledR} 2 \mathrm{NC}$ mini stacks, ThermoFisher Scientific) with iBlot@ 2 Gel temperature in phosphate-buffered saline $(\mathrm{pH} 7.4)$ containing $5 \%$ skim milk (BD biosciences, CA) to block nonspecific antibody binding. After $1 \mathrm{hr}$ incubation, membranes were individually

561 stained to detect E, prM and M proteins by using anti-DENV2 MHIAF at 1:2000, anti-DENV prM MAb $155-49$ at $0.5 \mu \mathrm{g} / \mathrm{ml}$ and mouse anti-M sera at $1: 25$, respectively, at $4^{\circ} \mathrm{C}$ overnight. Membranes were washed three times for 15 min each. DENV-specific bound immunoglobulin was recognized with HRP-labeled goat anti-mouse IgG (Jackson ImmunoReasearch, PA), and was visualized with ECL (enhanced chemiluminescent substrate, GE Healthcare, UK) according 
567 mini (GE Healthcare). However, M protein was visualized using TMB membrane peroxidase

568 substrate (KPL, MD) to avoid high background from ECL. imD2VLP and mD2VLP at weeks 0 and 4 at a dose of $4 \mu \mathrm{g} / 100 \mu \mathrm{L}$ PBS divided between the right and left quadriceps muscle. Mice were bled from the retro-orbital sinus at week 4 following the second injection, and individual serum specimens were evaluated for DENV-2 specific antibodies by ELISA and focus-reduction micro-neutralization test $(\mathrm{FR} \mu \mathrm{NT})$, as described in the following section. of non-immunized males with immunized females 11 weeks post initial vaccination were obtained for viral challenge. Immune sera were collected from immunized females 1 week prior

581 to mating to confirm the presence of the total $\mathrm{IgG}$ titer as well as the virus neutralization 582 antibody titer. Pups from unvaccinated females were used as the challenge control. ICR pups 583 from the designated groups were challenged individually through the intracranial route at 2 days 584 after birth with $10^{4}$ focus-forming units (FFU) which were equivalent to $141,61,11,1000$-fold 585 of $50 \%$ lethal doses (LD50) of DENV-1 (strain Hawaii), DENV-2 (strain 16681), DENV-3 586 (strain H87) and DENV-4 (strain BC71/94, kindly provided by one of the co-author, Dr. Chang 587 from US-CDC), respectively. Mouse survival was evaluated daily for up to 21 days. 
The neutralizing ability of the immune mouse sera for all serotypes of DENV was measured by focus-reduction micro neutralization test $(\mathrm{FR} \mu \mathrm{NT})$, as previously described(Galula et al., 2014). Briefly, $2.475 \times 10^{4}$ Vero cells/well were seeded onto flat-bottom 96-well Costar ${ }^{\circledR}$ cell culture plates (Corning Inc., Corning, NY, USA) and incubated for $16 \mathrm{hrs}$ overnight at $37^{\circ} \mathrm{C}$ with $5 \% \mathrm{CO}_{2}$. Pooled sera were initially diluted at 1:10, heat-inactivated for $30 \mathrm{~min}$ at $56^{\circ} \mathrm{C}$, titrated two-fold to a $40 \mu \mathrm{L}$ volume, and $320 \mathrm{pfu} / 40 \mu \mathrm{L}$ of DENV-1 to 4 was added to each dilution. The mixtures were then incubated for $1 \mathrm{hr}$ at $37^{\circ} \mathrm{C}$. After incubation, $25 \mu \mathrm{L}$ of the immune complexes were added in duplicates onto plates containing Vero cell monolayers. Plates were incubated for $1 \mathrm{hr}$ at $37^{\circ} \mathrm{C}$ with $5 \% \mathrm{CO}_{2}$ and rocked every 10 mins to allow infection. Overlay medium containing $1 \%$ methylcellulose (Sigma-Aldrich Inc., St. Louis, MO, USA) in DMEM with $2 \%$ FBS was added, and plates were incubated at $37^{\circ} \mathrm{C}$ with $5 \% \mathrm{CO}_{2}$. Forty-eight 601 hours later, plates were washed, fixed with $75 \%$ acetone in PBS and air-dried. Immunostaining 602 was performed by adding serotype-specific MHIAF at 1:600 in 5\% milk and 0.1\% PBST and 603 incubated for $60 \mathrm{~min}$ at $37^{\circ} \mathrm{C}$. Plates were washed and goat anti-mouse IgG-HRP at 1:100 in 5\% milk and $0.1 \%$ PBST was added; plates were incubated for $45 \mathrm{~min}$ at $37^{\circ} \mathrm{C}$. Infection foci were 605 visualized using a peroxidase substrate kit, Vector ${ }^{\circledR}$ VIP SK-4600 (Vector Laboratories, Inc., 606 Burlingame, CA, USA), following the manufacturer's instructions. FR $\mu$ NT titers were calculated 607 for each virus relative to a virus only control back-titration. Titers were determined as a $50 \%$ 608 reduction of infection foci $(\mathrm{FR} \mu \mathrm{NT} 50)$ and were modeled using a sigmoidal dose-response 609 (variable slope) formula. All values were determined from the average of two independent 
experiments. Target virus strains were: DENV-1, Hawaii; DENV-2, 16681; DENV-3, H87; and DENV-4, H241. For E-dimer, inter-domain-specific neutralizing antibodies, the recombinant parental DENV-2 strain PL046 and EDIII-swapped PL046cEDIII were used as target viruses. In the calculation of geometric mean titers (GMT) for graphic display and statistical analysis, a FR $\mu$ NT50 titer of $<10$ was represented with the value of 1 and 5, respectively.

\section{Generating hybridomas and MAb screening}

Hybridomas secreting anti-DENV antibodies were generated from the mD2VLP immunized mice according to a standard procedure(Kohler and Milstein, 1975), with slight modifications(Chen et al., 2007). First, the mD2VLP-immunized mouse was boosted with another $4 \mu \mathrm{g}$ of mD2VLP 24 weeks prior to terminal bleeding. At day 4 after the third immunization, splenocytes were harvested from the immunized mouse and fused with NSI/1Ag4-1 myeloma cells using an antibody delivery kit following manufacturer's recommendations (GenomONETM-CF HVJ Envelope Cell Fusion Kit, Gosmo Bio Co, ISK10 MA17). Fused cell pellets were suspended in DMEM supplemented with 15\% FBS, hypoxanthine-aminopterinthymidine medium, and hybridoma cloning factor (ICN, Aurora, OH). Hybridoma colonies were screened for secretion of MAbs by ELISA following the procedures as described above. However, the cell culture supernatant of C6/36 cells infected with DENV-2 virus at a multiplicity of infection (moi) equal to 1.0 was used as the antigens and the culture supernatant from each of the hybridoma colonies was used as the detecting antibody. Selected positive clones were subcloned by limiting dilution. Ascitic fluids were produced in pristane-primed BALB/c mice. Hybridoma cell lines were grown in DMEM with $10 \%$ heat inactivated FBS. MAbs were 
632 affinity purified with protein G Sepharose 4B gel, and the amount of each purified MAb was quantified by comparison with a known amount of IgG used in a standard ELISA. were collected from the spleens of BALB/c mice immunized twice at 4-week intervals with imD2VLP and mD2VLP. The D2VLP(+), B220 (+) and IgG1(+) splenic B cells were isolated by flow cytometry (FACSAria II), and single B cells were sorted into 96-well PCR plates 641 containing 4ul/well of ice-cold RNase-free water supplemented with 10mM DTT and 3U RNase 642 inhibitor (Promega). RT-PCR reactions were performed using the Qiagen OneStep RT-PCR kit 643 (Qiagen) at $50^{\circ} \mathrm{C}$ for $30 \mathrm{mins}$ and at $95^{\circ} \mathrm{C}$ for $15 \mathrm{~min}$ followed by 40 cycles of $95^{\circ} \mathrm{C}$ for $1 \mathrm{~min}$, $64455^{\circ} \mathrm{C}$ for $1 \mathrm{~min}$ and $72^{\circ} \mathrm{C} 1 \mathrm{~min}$, and a final incubation at $72^{\circ} \mathrm{C}$ for $5 \mathrm{~min}$, with the primers as 645 previously described. The nested PCR was performed using 2 ul of unpurified, first-round PCR 646 product at $95^{\circ} \mathrm{C}$ for 1 min followed by 40 cycles of $95^{\circ} \mathrm{C} 30 \mathrm{sec}, 57^{\circ} \mathrm{C}(\operatorname{IgH})$ or $45^{\circ} \mathrm{C}$ (Igk) or $64755^{\circ} \mathrm{C}(\operatorname{Ig} \lambda)$ for $30 \mathrm{sec}, 72^{\circ} \mathrm{C}$ for $45 \mathrm{sec}$, and incubated at $72^{\circ} \mathrm{C}$ for $10 \mathrm{~min}$ as previously described.

648 Aliquots of nested PCR products were sequenced and analyzed using IMGT/V-Quest 649 (http://www.imgt.org) to identify the highest homology gene loci of germ-line V, D and J genes. 650 Ig genes were then translated and aligned by CLUSTALW to define the clonally amplified Ig 651 genes. 
Immunogold labeling and transmission electron microscopy (TEM)

To detect mature DENV-2 VLPs (mD2VLPs) as spherical particles, $3 \mu \mathrm{L}$ of freshly prepared samples were adsorbed onto a glow-discharged nickel grid (EMS CF-200-Ni) for 20 mins, The grid containing the samples was incubated in 1\% BSA/PBS blocking buffer for 1 hour at room temperature. As soon as the excess liquid was removed with a filter paper, DENV-2specific MAb DB32-6 was used as the primary antibody (dilution 1:500), and was added to the samples for $2 \mathrm{hrs}$ at room temperature. Then the grid was washed with blocking buffer and incubated with 6-nm nanogold-conjugated Donkey anti-mouse IgG (Abcam, MA) (dilution 1:30) at room temperature for $1 \mathrm{hr}$. The excess liquid was removed with filter paper and fixed with $1 \%$ glutaraldehyde in PBS buffer (GA/PBS) for 10 mins. After fixing, excess liquid was removed using a filter paper. Finally, the sample was stained with $2 \%$ uranyl acetate (UA) for 1 min and air-dried. The immunogold labeling process was performed in a high humidity chamber. The immunogold labeled mD2VLPs were inspected by TEM. The images were taken by a JEM1400 electron transmission microscope at a magnification of 100,000x using a 4k x 4k Gatan 895 CCD camera. The diameters of particles were measured by ImageJ software.

\section{Cryo-EM and 3D reconstruction}

A freshly prepared dengue virus sample $(3 \mu \mathrm{l})$ was placed onto a glow-discharged Quatifoil 2/2 grid (Quatifoil GmbH, Germany), blotted with filter paper, and plunged into liquid nitrogen-cooled liquid ethane using Gatan CP3. Cryo-EM images were recorded with a JEM2100F using an accelerating voltage of $200 \mathrm{kV}$ and a magnification of $15,000 \mathrm{x}$ using a direct 
674 electron detector (DE-12 Camera System - Direct Electron, LP) with a $6 \mu \mathrm{m}$ pixel size

675 (corresponding to $\sim 4 \AA$ at the specimen level). The measured defocus values of these images

676 range from $-2 \mu \mathrm{m}$ to $-4.5 \mu \mathrm{m}$. The imaging electron dosage was $\sim 10 \mathrm{e}^{-} / \AA^{2}$.

Variations in size and shape were observed in the EM images (Figure S3A, B in

679 Supplementary Material). The irregularly-shaped particles were eliminated through visual

680 inspection, while the spherical particles were subjected to further image analyses. The particle

681 size analyses showed that the major peaks were located at $\sim 26 \mathrm{~nm}, \sim 31 \mathrm{~nm}$ and $\sim 36 \mathrm{~nm}$ diameter

682 size classes which were therefore denoted by small (' $S$ '), medium (' $M$ ') and large (' $L$ '). The

683 heterogeneity analyses by EMAN2(Tang et al., 2007) and XMIPP(Sorzano et al., 2004;de la

684 Rosa-Trevín et al., 2013) showed that all of the classes had distinct two layers and that the

685 particles classes with diameters of $31 \mathrm{~nm}$ had more prominent features than others (Figure S3B, C

686 in Supplementary Material). Taken together with the immunogold labeling results, we selected

687 the particles with size of $\sim 31 \mathrm{~nm}$ or further $3 \mathrm{D}$ reconstruction process. The structure

688 reconstruction processes were performed by EMAN2. There were 4,217 spherical particles with

689 the size of $\sim 31 \mathrm{~nm}$ which were included in the reconstruction process and the icosahedral

690 symmetry was imposed during the whole process. The reconstruction process ended when there

691 was no improvement achieved. The resolution of the final reconstructions was $13.1 \AA$ from a

692 Fourier shell correlation curve using the gold standard resolution estimate in EMAN2 (Figure S5

693 in Supplementary Material). Solvent accessible surface area (SASA) of individual amino acid

694 molecules on mD2VLP was calculated by POPS program(Cavallo et al., 2003;Fornili et al., 695 2012). 

mature dengue virus at 3.5A resolution (PDB ID: 3J27) using MODELLER 9v15(Eswar et al., 700 2007). The envelop proteins were fitted rigidly using the Fit-in-Map tool in UCSF Chimera, 701 when the CCC (Cross-Correlation Coefficient) score was maximized, the complete copies were 702 generated to follow the $\mathrm{T}=1$ arrangement with Multiscale Models using Icosahedral symmetry, 703 xyz 2-fold axes (VIPER).

709 were considered significant.

\section{Data and materials availability}

713 The cryo-EM density map of mD2VLP has been deposited to Electron Microscopy Data Bank

714 under accession number EMDB6926. All other relevant data are available from the authors on 715 request. 
718 Acknowledgments:

719 We thank Dr. Felix Rey for commenting on the structure of dengue VLP and Ann Hunt for 720 English editing.

Author contributions: WFS performed the characterization of D2VLP including Western blotting, ELISA, site-directed mutagenesis and mouse immunization. JHL conducted the Pitou

724 and solvent accessibility analysis. MYL, DYC, HCW and JUG produced and characterized the

725 NS1-splenocytes fused B cell lines, monoclonal antibody and single B-cell repertoire

726 characterization. WFS and YCW conducted VLP purification, mouse immunization experiments

727 and neutralization assays. JUG and $\mathrm{CHH}$ prepared the anti-M protein mouse polyclonal serum.

728 JJL and YLL prepared the parental and domain III-replaced recombinant DENV-2. SRC and

729 SRW prepared the cryo-EM samples, processed the cryo-EM images, fitted the protein

730 structures, and solved the cryo-EM structure. MTW, GJC and WFS mapped the $2 \mathrm{H} 2$ binding

731 epitopes. SRW, GJC and DYC supervised the studies and contributed to the writing of the paper.

733 Competing interests: All the authors declare that they have no competing interests. 
bioRxiv preprint doi: https://doi.org/10.1101/351700; this version posted June 20, 2018. The copyright holder for this preprint (which was not certified by peer review) is the author/funder, who has granted bioRxiv a license to display the preprint in perpetuity. It is made available under aCC-BY 4.0 International license.

735 Funding: This study was supported by Ministry of Science and Technology Taiwan (MOST

736 104-2320-B-006-027 and MOST 105-2320-B-006-017-MY3).

737

738

739 


\section{References}

Aguiar, M., Stollenwerk, N., and Halstead, S. (2016). The Impact of the Newly Licensed Dengue Vaccine in Endemic Countries. PLoS Negl Trop Dis 10, e0005179.

Allison, S., Stadler, K., Mandl, C., Kunz, C., and Heinz, F. (1995). Synthesis and secretion of recombinant tickborne encephalitis virus protein $\mathrm{E}$ in soluble and particulate form. $J$ Virol 69, 5816-5820.

Bachmann, M., Kalinke, U., Althage, A., Freer, G., Burkhart, C., Roost, H., Aguet, M., Hengartner, H., and Zinkernagel, R. (1997). The role of antibody concentration and avidity in antiviral protection. Science 276, 2024-2027.

Bhatt, S., Gething, P., Brady, O., Messina, J., Farlow, A., Moyes, C., Drake, J., Brownstein, J., Hoen, A., Sankoh, O., Myers, M., George, D., Jaenisch, T., Wint, G., Simmons, C., Scott, T., Farrar, J., and Hay, S. (2013). The global distribution and burden of dengue. Nature 496, 504-507.

Cavallo, L., Kleinjung, J., and Fraternali, F. (2003). POPS: A fast algorithm for solvent accessible surface areas at atomic and residue level. Nucleic Acids Res 31, 3364-3366.

Chambers, T.J., Hahn, C.S., Galler, R., and Rice, C.M. (1990). Flavivirus genome: organization, expression and replication. Annu. Rev. Microbiol. 44, 649-688.

Chang, G.-J.J., Hunt, A.R., Holmes, D.A., Springfield, T., Chiueh, T.S., Roehrig, J.T., and Gubler, D.J. (2003). Enhancing biosynthesis and secretion of premembrane and envelope proteins by the chimeric plasmid of dengue virus type 2 and Japanese encephalitis virus. Virology 306, 170-180.

Chang, G., Hunt, A., and Davis, B. (2000). A single intramuscular injection of recombinant plasmid DNA induces protective immunity and prevents Japanese encephalitis in mice. J Virol 74, 4244-4252.

Chen, H., Liu, S., Li, Y., Liu, H., Tsai, J., Chiang, C., Chen, M., Hwang, C., Huang, C., Hu, H., Chung, H., Wu, S., Chong, P., Leng, C., and Pan, C. (2013). A consensus envelope protein domain III can induce neutralizing antibody responses against serotype 2 of dengue virus in non-human primates. Arch Virol 158, 1523-1531.

Chen, Y., Huang, H., Lin, C., Chen, Y., King, C., and Wu, H. (2007). Generation and characterization of monoclonal antibodies against dengue virus type 1 for epitope mapping and serological detection by epitope-based peptide antigens. Clin Vaccine Immunol 14, 404-411.

Cherrier, M., Kaufmann, B., Nybakken, G., Lok, S., Warren, J., Chen, B., Nelson, C., Kostyuchenko, V., Holdaway, H., Chipman, P., Kuhn, R., Diamond, M., Rossmann, M., and Fremont, D. (2009). Structural basis for the preferential recognition of immature flaviviruses by a fusion-loop antibody. EMBO J 28, 3269-3276.

Crill, W., Hughes, H., Delorey, M., and Chang, G. (2009). Humoral immune responses of dengue fever patients using epitope-specific serotype-2 virus-like particle antigens. PLoS One 4, e4991.

Crill, W.D., Hughes, H.R., Trainor, N.B., Davis, B.S., Whitney, M.T., and Chang, G.J. (2012). Sculpting humoral immunity through dengue vaccination to enhance protective immunity. Front Immunol 3, 334.

Crowe, J.J. (2017). Principles of Broad and Potent Antiviral Human Antibodies: Insights for Vaccine Design. Cell Host Microbe 22, 193-206.

Da Silva Voorham, J., Rodenhuis-Zybert, I., Ayala Nuñez, N., Colpitts, T., Van Der Ende-Metselaar, H., Fikrig, E., Diamond, M., Wilschut, J., and Smit, J. (2012). Antibodies against the envelope glycoprotein promote infectivity of immature dengue virus serotype 2. PLoS One 7, e29957.

De Alwis, R., Beltramello, M., Messer, W., Sukupolvi-Petty, S., Wahala, W., Kraus, A., Olivarez, N., Pham, Q., Brien, J., Tsai, W., Wang, W., Halstead, S., Kliks, S., Diamond, M., Baric, R., Lanzavecchia, A., Sallusto, F., and De Silva, A. (2011). In-depth analysis of the antibody response of individuals exposed to primary dengue virus infection. PLoS Negl Trop Dis 5, e1188.

De La Rosa-Trevín, J., Otón, J., Marabini, R., Zaldívar, A., Vargas, J., Carazo, J., and Sorzano, C. (2013). Xmipp 3.0: an improved software suite for image processing in electron microscopy. J Struct Biol 184, 321-328.

Dejnirattisai, W., Jumnainsong, A., Onsirisakul, N., Fitton, P., Vasanawathana, S., Limpitikul, W., Puttikhunt, C., Edwards, C., Duangchinda, T., Supasa, S., Chawansuntati, K., Malasit, P., Mongkolsapaya, J., and Screaton, G. (2010). Cross-reacting antibodies enhance dengue virus infection in humans. Science 328, 745-748.

Dejnirattisai, W., Wongwiwat, W., Supasa, S., Zhang, X., Dai, X., Rouvinski, A., Jumnainsong, A., Edwards, C., Quyen, N., Duangchinda, T., Grimes, J., Tsai, W., Lai, C., Wang, W., Malasit, P., Farrar, J., Simmons, C., Zhou, Z., Rey, F., Mongkolsapaya, J., and Screaton, G. (2015). A new class of highly potent, broadly neutralizing antibodies isolated from viremic patients infected with dengue virus. Nat Immunol 16, $170-177$. 
Eswar, N., Webb, B., Marti-Renom, M., Madhusudhan, M., Eramian, D., Shen, M., Pieper, U., and Sali, A. (2007). Comparative protein structure modeling using MODELLER. Curr Protoc Protein Sci Chapter 2.

Ferlenghi, I., Clarke, M., Ruttan, T., Allison, S., Schalich, J., Heinz, F., Harrison, S., Rey, F., and Fuller, S. (2001). Molecular organization of a recombinant subviral particle from tick-borne encephalitis virus. Mol Cell 7, 593-602.

Fibriansah, G., Ibarra, K., Ng, T., Smith, S., Tan, J., Lim, X., Ooi, J., Kostyuchenko, V., Wang, J., De Silva, A., Harris, E., Crowe, J.J., and Lok, S. (2015a). DENGUE VIRUS. Cryo-EM structure of an antibody that neutralizes dengue virus type 2 by locking E protein dimers. Science 349, 88-91.

Fibriansah, G., Tan, J., Smith, S., De Alwis, R., Ng, T., Kostyuchenko, V., Jadi, R., Kukkaro, P., De Silva, A., Crowe, J., and Lok, S. (2015b). A highly potent human antibody neutralizes dengue virus serotype 3 by binding across three surface proteins. Nat Commun 6.

Flipse, J., and Smit, J. (2015). The Complexity of a Dengue Vaccine: A Review of the Human Antibody Response. PLoS Negl Trop Dis 9, e0003749.

Fornili, A., Autore, F., Chakroun, N., Martinez, P., and Fraternali, F. (2012). Protein-water interactions in MD simulations: POPS/POPSCOMP solvent accessibility analysis, solvation forces and hydration sites. Methods Mol Biol 819, 375-392.

Galula, J.U., Shen, W.F., Chuang, S.T., Chang, G.J., and Chao, D.Y. (2014). Virus-like particle secretion and genotype-dependent immunogenicity of dengue virus serotype 2 DNA vaccine. J Virol 88, 10813-10830.

Guy, B., and Jackson, N. (2016). Dengue vaccine: hypotheses to understand CYD-TDV-induced protection. Nat Rev Microbiol 14, 45-54.

Halstead, S. (2017). Dengvaxia sensitizes seronegatives to vaccine enhanced disease regardless of age. Vaccine 35, 6355-6358.

Henein, S., Swanstrom, J., Byers, A., Moser, J., Shaik, S., Bonaparte, M., Jackson, N., Guy, B., Baric, R., and De Silva, A. (2017). Dissecting Antibodies Induced by a Chimeric Yellow Fever-Dengue, Live-Attenuated, Tetravalent Dengue Vaccine (CYD-TDV) in Naive and Dengue-Exposed Individuals. J Infect Dis 215, 351-358.

Huang, K., Yang, Y., Lin, Y., Huang, J., Liu, H., Yeh, T., Chen, S., Liu, C., and Lei, H. (2006). The dual-specific binding of dengue virus and target cells for the antibody-dependent enhancement of dengue virus infection. J Immunol 76, 2825-2832.

Hughes, H., Crill, W., and Chang, G. (2012). Manipulation of immunodominant dengue virus E protein epitopes reduces potential antibody-dependent enhancement. Virol J 9, 115.

Junjhon, J., Edwards, T., Utaipat, U., Bowman, V., Holdaway, H., Zhang, W., Keelapang, P., Puttikhunt, C., Perera, R., Chipman, P., Kasinrerk, W., Malasit, P., Kuhn, R., and Sittisombut, N. (2010). Influence of pr-M cleavage on the heterogeneity of extracellular dengue virus particles. J Virol 84, 8353-8358.

Katzelnick, L., Coloma, J., and Harris, E. (2017). Dengue: knowledge gaps, unmet needs, and research priorities. Lancet Infect Dis 17, e88-e100.

Keelapang, P., Nitatpattana, N., Suphatrakul, A., Punyahathaikul, S., Sriburi, R., Pulmanausahakul, R., Pichyangkul, S., Malasit, P., Yoksan, S., and Sittisombut, N. (2013). Generation and preclinical evaluation of a DENV$1 / 2$ prM+E chimeric live attenuated vaccine candidate with enhanced prM cleavage. Vaccine 31, 51345140.

Kirkpatrick, B., Whitehead, S., Pierce, K., Tibery, C., Grier, P., Hynes, N., Larsson, C., Sabundayo, B., Talaat, K., Janiak, A., Carmolli, M., Luke, C., Diehl, S., and Durbin, A. (2016). The live attenuated dengue vaccine TV003 elicits complete protection against dengue in a human challenge model. Sci Transl Med 8, $330 \mathrm{ra336.}$

Kohler, G., and Milstein, C. (1975). Continuous cultures of fused cells secreting antibody of predefined specificity. Nature 256, 495-497.

Kostyuchenko, V., Zhang, Q., Tan, J., Ng, T., and Lok, S. (2013). Immature and mature dengue serotype 1 virus structures provide insight into the maturation process. J Virol 87, 7700-7707.

Li, L., Lok, S., Yu, I., Zhang, Y., Kuhn, R., Chen, J., and Rossmann, M. (2008). The flavivirus precursor membraneenvelope protein complex: structure and maturation. Science 319, 1830-1834.

Li, P., Liao, M., Cheng, P., Liang, J., Liu, I., Chiu, C., Lin, Y., Chang, G., and Wu, H. (2012). Development of a humanized antibody with high therapeutic potential against dengue virus type 2. PLoS Negl Trop Dis 6, e1636.

Liang, J., Liao, C., Liao, J., Lee, Y., and Lin, Y. (2009). A Japanese encephalitis virus vaccine candidate strain is attenuated by decreasing its interferon antagonistic ability. Vaccine 27, 2746-2754. 
Lindenbach, B.D., and Rice, C.M. "Flaviviridae: The viruses and their replication." in Fields' Virology, D. M. Knipe and P. M. Howley, Eds. (Lippincott Williams \& Wilkins, Philadelphia, ed. 4, 2001). pp.991-1110, .

Lok, S. (2016). The interplay of dengue virus morphological diversity and human antibodies. Trends Microbiol 24, 284-293.

Lok, S.M., Kostyuchenko, V., Nybakken, G.E., Holdaway, H.A., Battisti, A.J., Sukupolvi-Petty, S., Sedlak, D., Fremont, D.H., Chipman, P.R., Roehrig, J.T., Diamond, M.S., Kuhn, R.J., and Rossmann, M.G. (2008). Binding of a neutralizing antibody to dengue virus alters the arrangement of surface glycoproteins. Nat Struct Mol Biol 15, 312-317.

Metz, S., Gallichotte, E., Brackbill, A., Premkumar, L., Miley, M., Baric, R., and De Silva, A. (2017). In Vitro Assembly and Stabilization of Dengue and Zika Virus Envelope Protein Homo-Dimers. Sci Rep 7, 4524.

Metz, S., Thomas, A., White, L., Stoops, M., Corten, M., Hannemann, H., and De Silva, A. (218). Dengue virus-like particles mimic the antigenic properties of the infectious dengue virus envelope. Virol J 15, 60.

Modis, Y., Ogata, S., Clements, D., and Harrison, S. (2003). A ligand-binding pocket in the dengue virus envelope glycoprotein. Proc Natl Acad Sci 100, 6986-6991.

Moodie, Z., Juraska, M., Huang, Y., Zhuang, Y., Fong, Y., Carpp, L., Self, S., Chambonneau, L., Small, R., Jackson, N., Noriega, F., and Gilbert, P. (2018). Neutralizing Antibody Correlates Analysis of Tetravalent Dengue Vaccine Efficacy Trials in Asia and Latin America. J Infect Dis 217, 742-753.

Nelson, S., Jost, C., Xu, Q., Ess, J., Martin, J., Oliphant, T., Whitehead, S., Durbin, A., Graham, B., Diamond, M., and Pierson, T. (2008). Maturation of West Nile virus modulates sensitivity to antibody-mediated neutralization. PLoS Pathog 4, e1000060.

Pierson, T., and Diamond, M. (2012). Degrees of maturity: the complex structure and biology of flaviviruses. Curr Opin Virol 2, 168-175.

Pierson, T., Fremont, D., Kuhn, R., and Diamond, M. (2008). Structural insights into the mechanisms of antibodymediated neutralization of flavivirus infection: implications for vaccine development. Cell Host Microbe 4, 229-238.

Purdy, D., and Chang, G. (2005). Secretion of noninfectious dengue virus-like particles and identification of amino acids in the stem region involved in intracellular retention of envelope protein. Virology 333, 239-250.

Rey, F., Stiasny, K., Vaney, M., Dellarole, M., and Heinz, F. (2018). The bright and the dark side of human antibody responses to flaviviruses: lessons for vaccine design. EMBO Rep 19, 206-224.

Rodenhuis-Zybert, I., Da Silva Voorham, J., Torres, S., Van De Pol, D., and Smit, J. (2015). Antibodies against immature virions are not a discriminating factor for dengue disease severity. PLoS Negl Trop Dis 9, e0003564.

Rodenhuis-Zybert, I., Moesker, B., Da Silva Voorham, J., Van Der Ende-Metselaar, H., Diamond, M., Wilschut, J., and Smit, J. (2011). A fusion-loop antibody enhances the infectious properties of immature flavivirus particles. J Virol 85, 11800-11808.

Rodenhuis-Zybert, I., Van Der Schaar, H., Da Silva Voorham, J., Van Der Ende-Metselaar, H., Lei, H., Wilschut, J., and Smit, J. (2010). Immature dengue virus: a veiled pathogen? PLoS Pathog 6, e1000718.

Roehrig, J.T. (2003). Antigenic structure of flavivirus proteins. Adv Virus Res. 59, 141-175.

Rouvinski, A., Dejnirattisai, W., Guardado-Calvo, P., Vaney, M., Sharma, A., Duquerroy, S., Supasa, P., Wongwiwat, W., Haouz, A., Barba-Spaeth, G., Mongkolsapaya, J., Rey, F., and Screaton, G. (2017). Covalently linked dengue virus envelope glycoprotein dimers reduce exposure of the immunodominant fusion loop epitope. Nat Commun 8, 15411.

Rouvinski, A., Guardado-Calvo, P., Barba-Spaeth, G., Duquerroy, S., Vaney, M., Kikuti, C., Navarro Sanchez, M., Dejnirattisai, W., Wongwiwat, W., Haouz, A., Girard-Blanc, C., Petres, S., Shepard, W., Desprès, P., Arenzana-Seisdedos, F., Dussart, P., Mongkolsapaya, J., Screaton, G., and Rey, F. (2015). Recognition determinants of broadly neutralizing human antibodies against dengue viruses. Nature 520, 109-113.

Schägger, H. (2006). Tricine-SDS-PAGE. Nat Protoc 1, 16-22.

Sorzano, C., Marabini, R., Velázquez-Muriel, J., Bilbao-Castro, J., Scheres, S., Carazo, J., and Pascual-Montano, A. (2004). XMIPP: a new generation of an open-source image processing package for electron microscopy. $J$ Struct Biol 148, 194-204.

Suphatrakul, A., Yasanga, T., Keelapang, P., Sriburi, R., Roytrakul, T., Pulmanausahakul, R., Utaipat, U., Kawilapan, Y., Puttikhunt, C., Kasinrerk, W., Yoksan, S., Auewarakul, P., Malasit, P., Charoensri, N., and Sittisombut, N. (2015). Generation and preclinical immunogenicity study of dengue type 2 virus-like particles derived from stably transfected mosquito cells. Vaccine 33, 5613-5622. 
Tang, G., Peng, L., Baldwin, P., Mann, D., Jiang, W., Rees, I., and Ludtke, S. (2007). EMAN2: an extensible image processing suite for electron microscopy. J Struct Biol 157, 38-46.

Tian, S., Huajun, W., and Wu, J. (2012). Computational prediction of furin cleavage sites by a hybrid method and understanding mechanism underlying diseases. Sci Rep 2, 261.

Tiller, T., Busse, C., and Wardemann, H. (2009). Cloning and expression of murine Ig genes from single B cells. $J$ Immunol Methods 350, 183-193.

Tsai, W., Lai, C., Wu, Y., Lin, H., Edwards, C., Jumnainsong, A., Kliks, S., Halstead, S., Mongkolsapaya, J., Screaton, G., and Wang, W. (2013). High-avidity and potently neutralizing cross-reactive human monoclonal antibodies derived from secondary dengue virus infection. J Virol 87, 12562-12575.

Wang, Z., Li, L., Pennington, J., Sheng, J., Yap, M., Plevka, P., Meng, G., Sun, L., Jiang, W., and Rossmann, M. (2013). Obstruction of dengue virus maturation by Fab fragments of the 2H2 antibody. J Virol 87, 89098915.

Wichmann, O., Vannice, K., Asturias, E., De Albuquerque Luna, E., Longini, I., Lopez, A., Smith, P., Tissera, H., Yoon, I., and Hombach, J. (2017). Live-attenuated tetravalent dengue vaccines: The needs and challenges of post-licensure evaluation of vaccine safety and effectiveness. Vaccine 35, 5535-5542.

World Health Organization (2017). Dengue vaccine: WHO position paper, July 2016 - recommendations. Vaccine 35, 1200-1201.

Yang, Y., Meng, Y., Halloran, M., and Longini, I. (2018). Dependency of Vaccine Efficacy on Preexposure and Age: A Closer Look at a Tetravalent Dengue Vaccine. Clin Infect Dis 66, 178-184.

Zhang, S., Tan, H., Hanson, B., and Ooi, E. (2010). A simple method for Alexa Fluor dye labelling of dengue virus. J Virol Methods 167, 172-177.

Zhang, X., Ge, P., Yu, X., Brannan, J., Bi, G., Zhang, Q., Schein, S., and Zhou, Z. (2013a). Cryo-EM structure of the mature dengue virus at 3.5-A resolution. Nat Struct Mol Biol 20, 105-110.

Zhang, X., Sheng, J., Austin, S., Hoornweg, T., Smit, J., Kuhn, R., Diamond, M., and Rossmann, M. (2015). Structure of acidic $\mathrm{pH}$ dengue virus showing the fusogenic glycoprotein trimers. J Virol 89, 743-750.

Zhang, X., Sheng, J., Plevka, P., Kuhn, R., and Diamond, M. (2013b). Dengue structure differs at the temperatures of its human and mosquito hosts. Proc Natl Acad Sci U S A 110, 6795-6799. 
A

1

9192 166

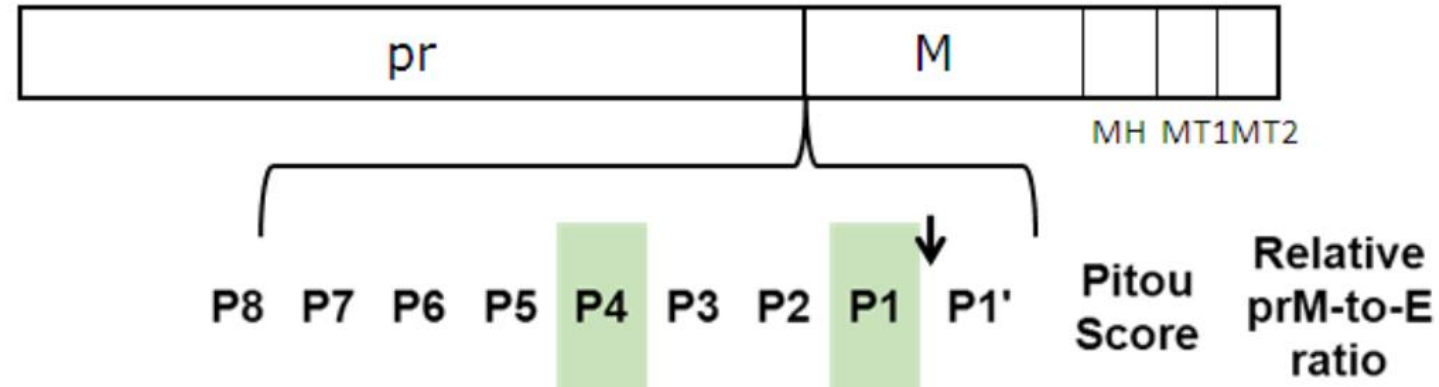

\section{DENV serocomplex}

DENV-2

$\begin{array}{lllll}G & E & H & R & R\end{array}$

DENV-1

G $E$ H R

DENV-3

G $E$ H $R$ R

$\begin{array}{llll}E & K & R & S\end{array}$

S $\quad 11.01$

82.3

DENV-4

G E R

$\mathrm{R}$

$\begin{array}{lllll}R & D & K & R & S\end{array}$

6.90

ND

JEV serocomplex

JEV

R H S K

SLEV

G $H \quad S \quad R$

$R$

D $K$ R

S $\quad 6.87$

ND

WNV

R $H \quad S$

Other serocomplex

TBEV

YFV

CFAV

Zika

mD2VLP

imD2VLP

B
$\begin{array}{lllllllll}Q & E & G & S & R & T & R & R & S \\ G & R & S & R & R & S & R & R & A \\ V & V & K & K & R & E & K & R & S \\ G & E & A & R & R & S & R & R & A \\ V & V & K & K & R & S & K & R & S \\ \text { G } & E & H & R & R & E & S & T & S\end{array}$

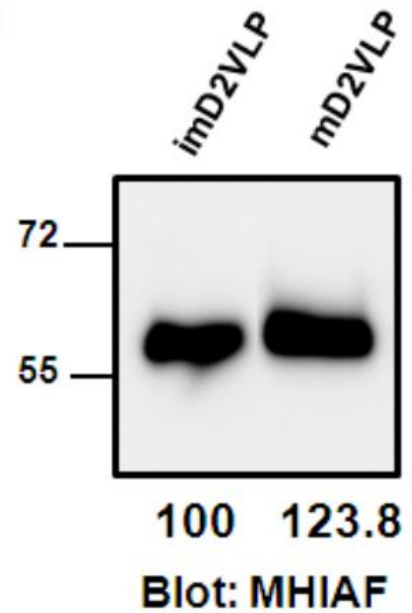

Blot: MHIAF
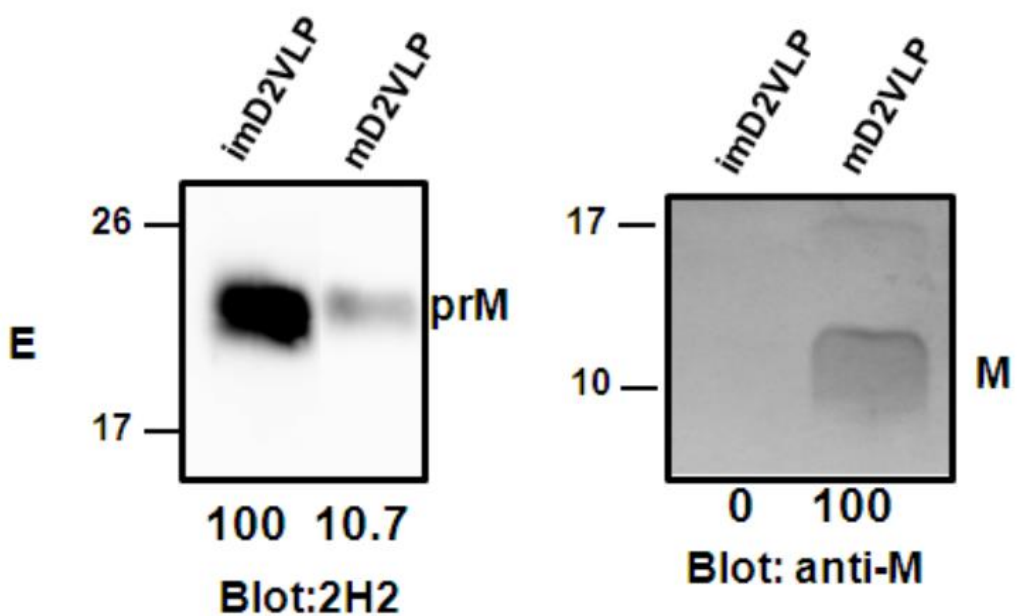
Fig. 1. Comparison of the prM junction cleavage efficiency among different DENV-2 virus-like particles (D2VLPs). (A) Schematic drawing of the prM protein. The C-terminal of the prM protein contains an $\alpha$-helical domain $(\mathrm{MH})$ in the stem region, followed by two transmembrane domains (MT1 and MT2). Numbers refer to the position of the amino acids in the polyprotein starting at the first amino acid of prM according to DENV-2 (NP_056776). Single letter designations of amino acid sequence alignment of representative strains from different serocomplexes of flaviviruses at the prM junction site includes dengue virus serotypes 1-4 (DENV-1 to DENV-4), Japanese encephalitis virus (JEV), St. Louis encephalitis virus (SLEV), West Nile virus (WNV), tick-borne encephalitis virus (TBEV), yellow fever virus (YFV), cell-fusion agent virus (CFAV), Zika virus (ZKV), immature DENV-2 VLP (imD2VLP) and mature DENV-2 VLP (mD2VLP). Numbers with P in the beginning refer to the positions of the amino acid relative to the prM cleavage site in the proximal direction (without apostrophe) and the distal direction (with apostrophe). Protein sequences were aligned, with the key P1 and P4 positions within the furin cleavage sites highlighted. The arrow indicates the prM cleavage site. The amino acids in red indicate the residues different from DENV-2. The PiTou 2.0 furin cleavage prediction scores are shown on the right for each sequence and the higher scores indicate the higher efficiency of furin cleavage. The relative quantity of prM and E of the wild-type and mutant DENV-2 VLP with P1-8 replacement (from other flaviviruses as shown) was measured by ELISA using MAb 3H5 (specific to E domain III of DENV-2) and MAb 155-49 (specific to DENV prM). The relative prM-to-E ratios were calculated by absorbance for prM/absorbance for $\mathrm{E}$ protein with reference to imD2VLP, whose pr portion was set as $100 \%$ uncleaved, as shown on the right. ND: not determined. Data are presented as means from three representative ELISA experiments with two replicates. (B) Culture supernatants of mD2VLP and imD2VLPs were collected and purified after electroporation with the respective plasmids. Five micrograms of proteins were loaded onto a $12 \%$ non-reducing Tricine-SDS-PAGE. E, prM and M proteins were 
954 assayed by Western blot using mouse hyper-immune ascitic fluids (MHIAF, 1:2000), MAb $2 \mathrm{H} 2$ (0.5

$955 \mu \mathrm{g} / \mathrm{mL})$ and anti-M protein mouse sera (1:25), respectively. E and prM proteins were visualized with

956 enhanced chemiluminesence (ECL); however, M protein was visualized by TMB substrate to avoid

957 high background. The number below each blot shows the relative densitometric quantification of E,

958 prM and M protein bands by Bio-1D software.

959

960 
A
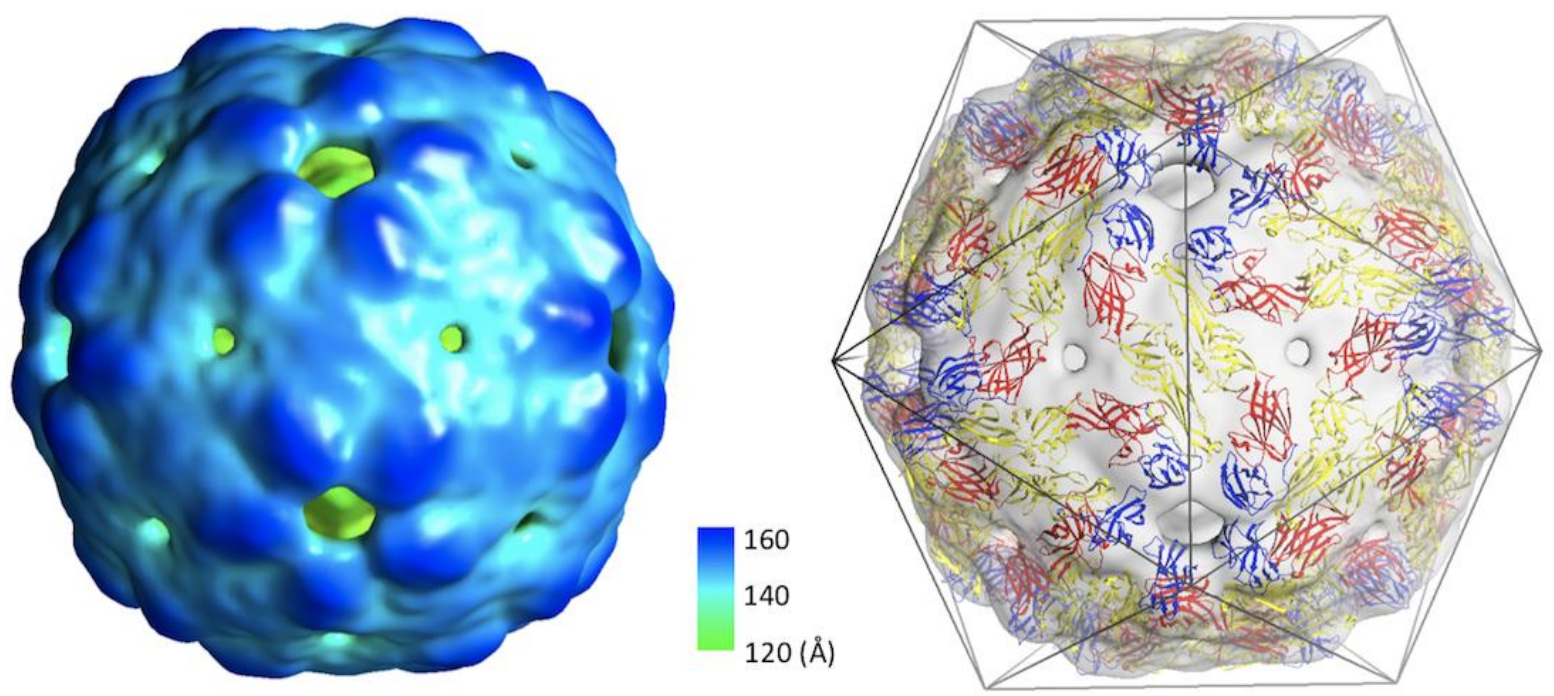

B

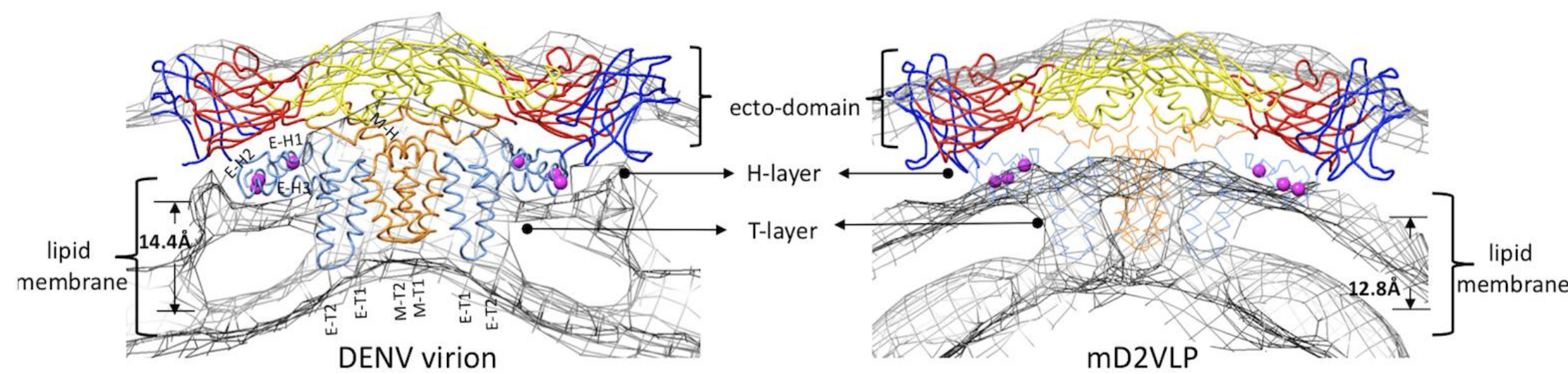



EM map of the DENV VLPs (left panel) were presented with the radial color-code indicated (left), the size of the particle is $31 \mathrm{~nm}$. Fitting of atomic E, M surface protein (PDB: 3J27) into cryo-EM density map (right) showed that the VLP has 60 copies of E in a T=1 arrangement. The density map was shown as a transparent volume rendering into which was fitted the backbone structures of the E ecto-domain. The domains I, II, and III were highlighted in red, yellow and blue, respectively. The cage indicated the icosahedral symmetry. (B) The cross section showed the fitted E:M:M:E heterotetramer (PDB: 3J27) into DENV virion (left) and into mD2VLP (right). The map density was in mesh presentation. The atomic model of E:M:M:E heterotetramer was showed in ribbon. Domain definition of dengue $\mathrm{E}$ was the same as the previous description, the transmembrane domain of $\mathrm{E}$ was colored as light blue, the $\mathrm{M}$ protein was in orange color. It was clear that the density of H-layer which is composed of E-H1 to E-H3 and M-H was more solid while the density in T-layer which contains E-T1, E-T2, M-T1 and M-T2 was weak in VLP than in virion. The residues at 398, 401 and 412 in E-H1 of JEV sequence which were proved to play important role in promoting extracellular secretion ${ }^{28}$ were shown as magenta spheres. The transmembrane, perimembrane helices and lipid bilayer were labelled, the critical measurements were also shown.

975 
A

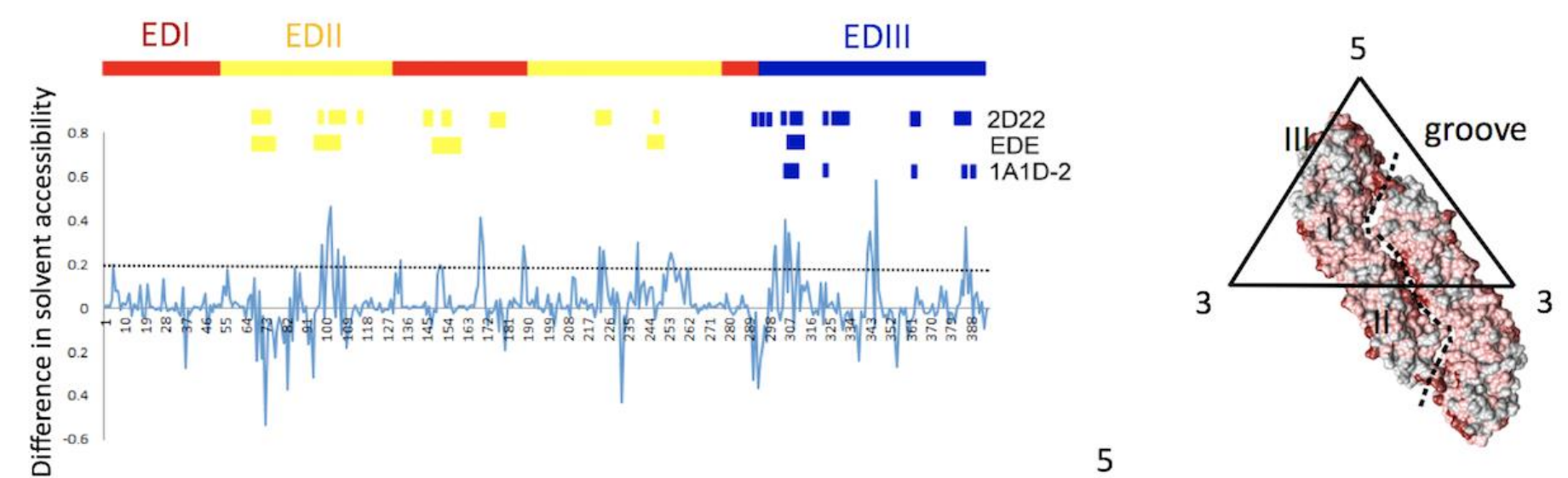

B

- Highly exposed residues 1A1D-2 binding epitopes
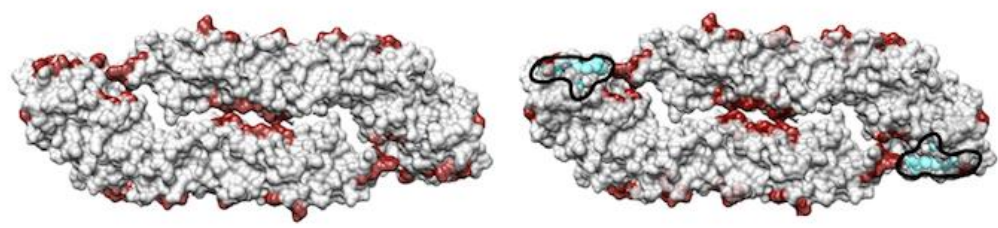

2D22 binding epitopes

- EDE binding epitopes
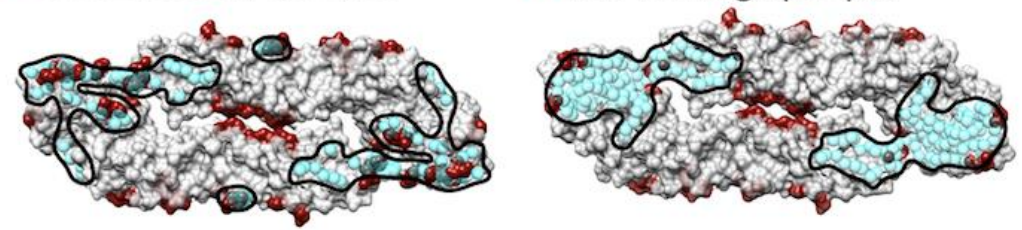

C
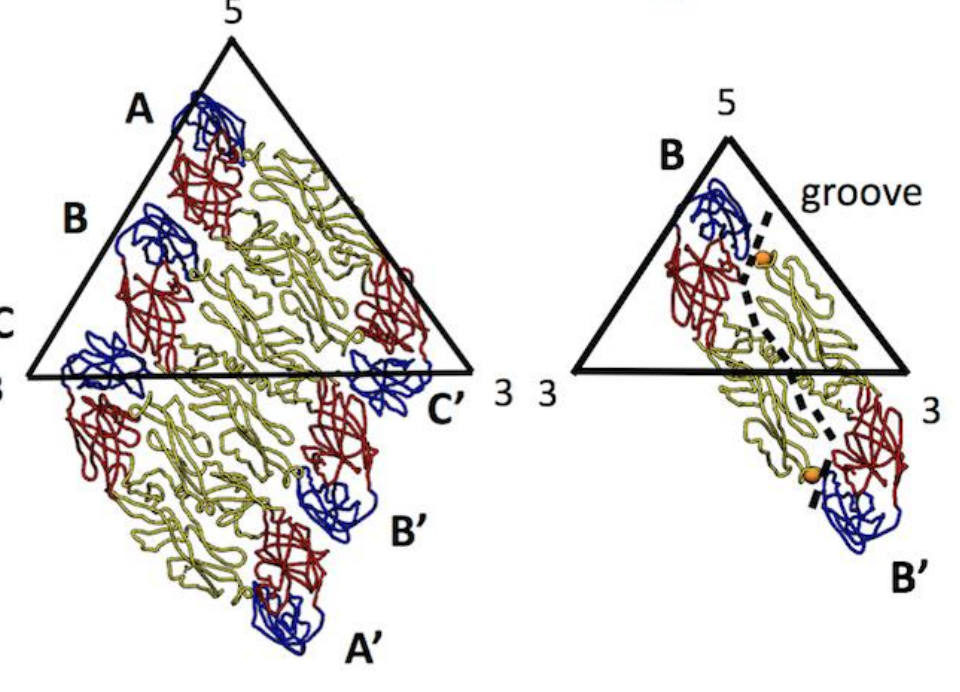
Figure 3. Solvent accessibility of dengue virus serotype 2 virus soluble envelope (sE) protein (A, left) The plot of difference in relative solvent-accessible surface area ( $\Delta \%$ SASA). A positive value of $\%$ SASA meant the residue became more exposed when the particle assembly shifts from virion (3 copies of E dimers per icosahedral asymmetric unit) to VLP (1 copy of E dimers per icosahedral asymmetric unit). The black dash line indicated the $\Delta \% \mathrm{SASA} \geq 0.2$ which was defined as highly exposed residues in VLP comparing to virion. The high positive values which were focused in the peptide regions such as the fusion loop peptide (including amino acid (aa) residues ranging from 100-110), aa 169-170 at domain I, aa 222-226, aa 239 and aa 251-262 at domain II as well as A strand of domain III (aa 300-308), the cd loop of domain III (aa 342-348) and G strand (aa 386-388) around the 5-fold openings. The residues interacting with MAb 1A1D-2 $2^{30}$, including residues 305-312, 352, 364, 388 and 390; the residues interacting with MAb 2D22 ${ }^{31}$, including residues 67-72, 99, 101-104, 113, 177-180, 225-227, 247, 328, 384-386 (Heavy chain); 148-149, 153-155, 291-293, 295, 298, 299, 307, 309-310, 325, 327, 362-363 (light chain) and the residues interacting with human MAb EDE antibodies ${ }^{19}$, including aa residues $67-74,97-106,148-159,246-249$ and 307-314 were indicated. (A, right) The high positive peaks $(\Delta \%$ SASA $\geq 0.2)$, low positive peaks $(\Delta \%$ SASA between 0 and 0.2$)$ and negative peaks $(\Delta \%$ SASA $\leq 0)$ in the plot were colored by dark red, deem red and grey in the E dimer surface rendering. The groove located within E-dimer interface was outlined. (B) The highly exposed residues $(\Delta \% \mathrm{SASA} \geq 0.2)$ which were colored by dark red were shown in the surface rendered E-dimer. The residues in $\mathrm{E}$ interacting with MAb 1A1D-2, 2D22 and EDE were in cyan spheres showing that they were highly exposed on the m2DVLP surface. Importantly, the binding footprints of the three antibodies were highly overlapping with footprint of highly exposed residues in 
m2DVLP, and formed a neutralization sensitive patch on m2DVLPs. The areas of the interacting epitopes are circled by black lines.

994 (C) The E protein forming the rafts in virion were shown in the left panel where the three individual E proteins in the asymmetric unit 995 are labeled A, B, and C of the E proteins, in the neighboring asymmetric unit are labeled A', B', and C'. The icosahedral 2-fold E protein dimers (B and B') in m2DVLP have moved apart from each other causing the groove (right). The aa 101 which was 997 responsible for DM25-3 antibody binding were shown in orange spheres. 
DI/II

DIII

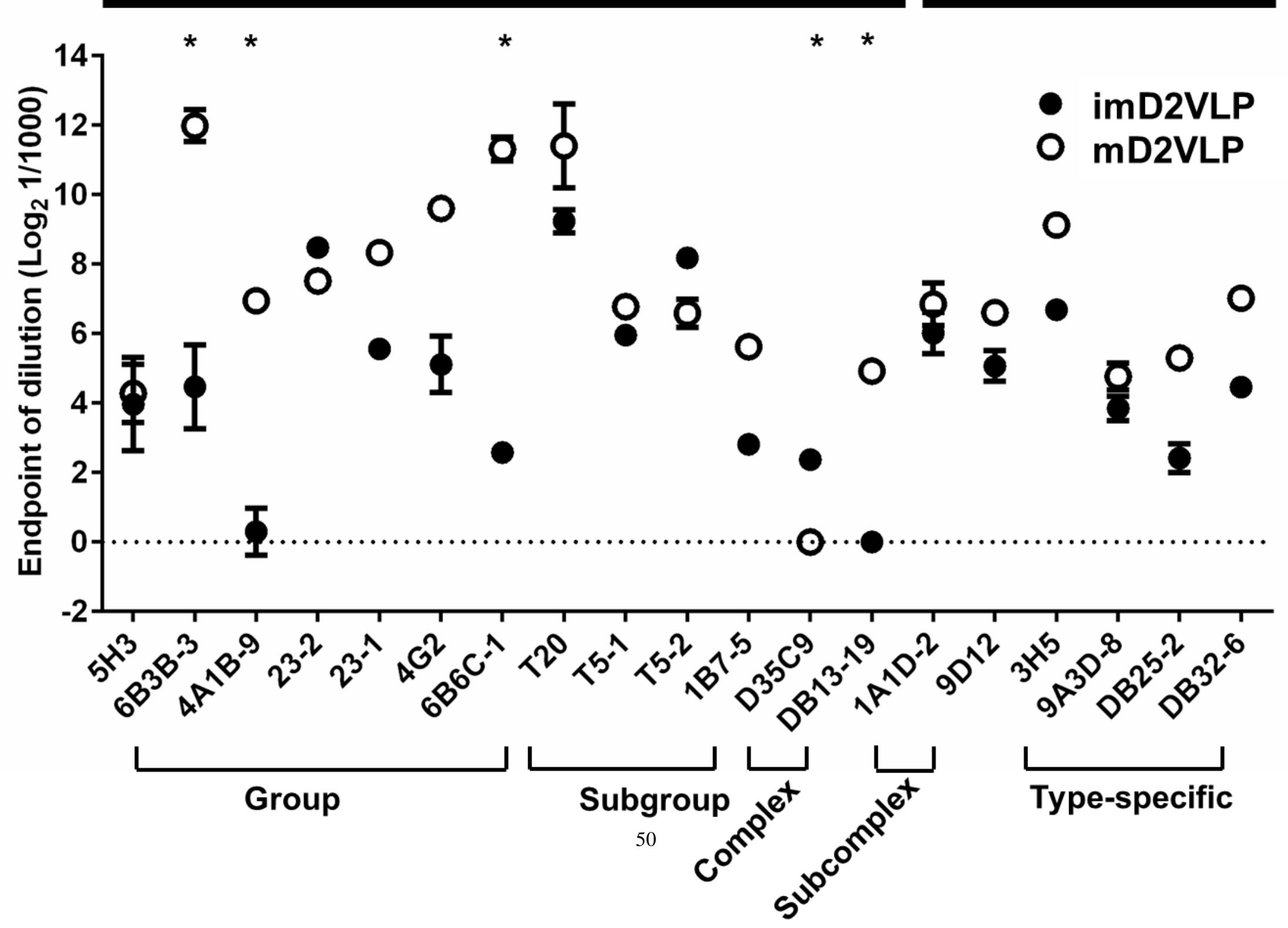


1001 Fig 4. Summary of binding activity of a panel of anti-E monoclonal antibodies (Mabs) to mD2VLP and imD2VLP. The binding activity of each Mab to 1002 two types D2VLP was expressed as means of endpoint dilution with standard deviations from duplicates of representative experiments of three. The 1003 dash line represents the minimum dilution of Mab used in the experiment. The asterisk symbol represents statistical significance between mD2VLP and imD2VLP in antibody dilution reaching endpoint with p-value $<0.05$

1005 

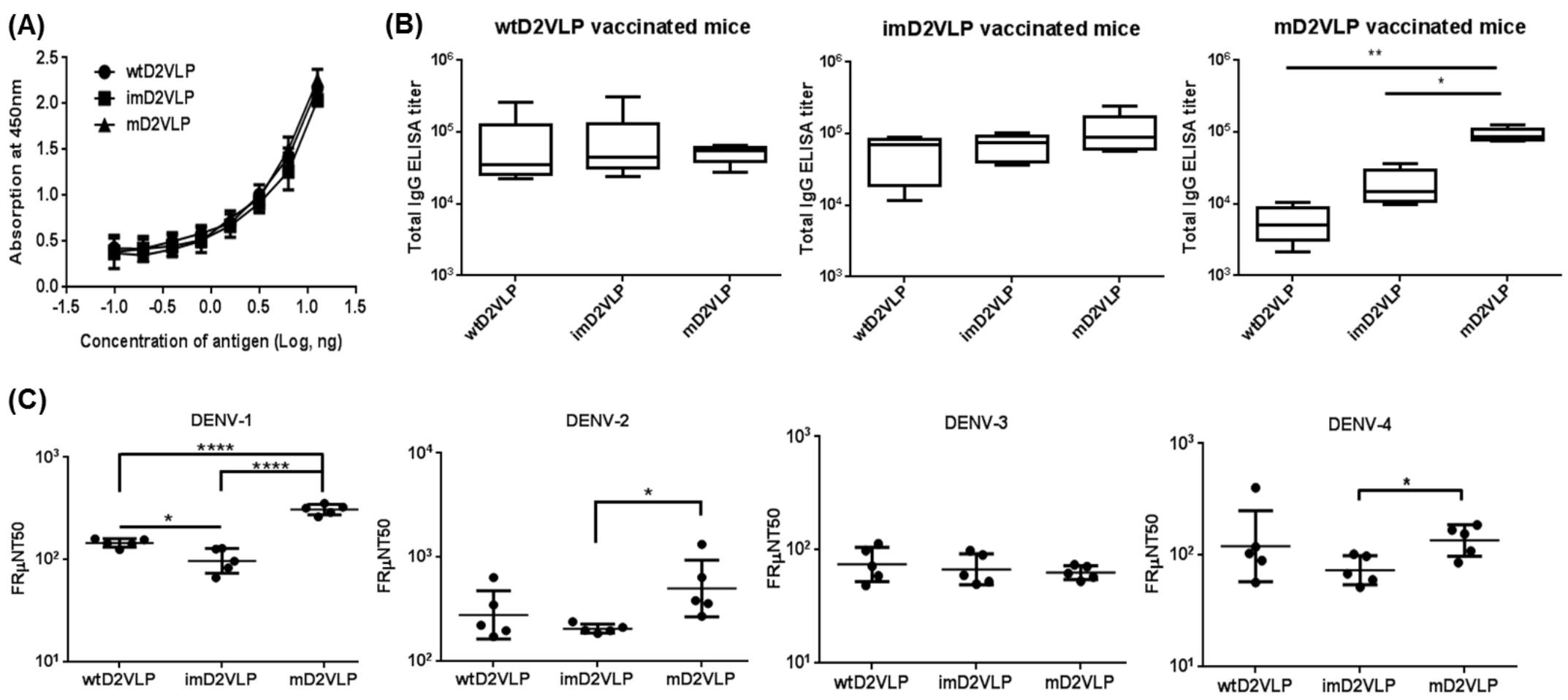

(D)

wtD2VLP vaccinated mice
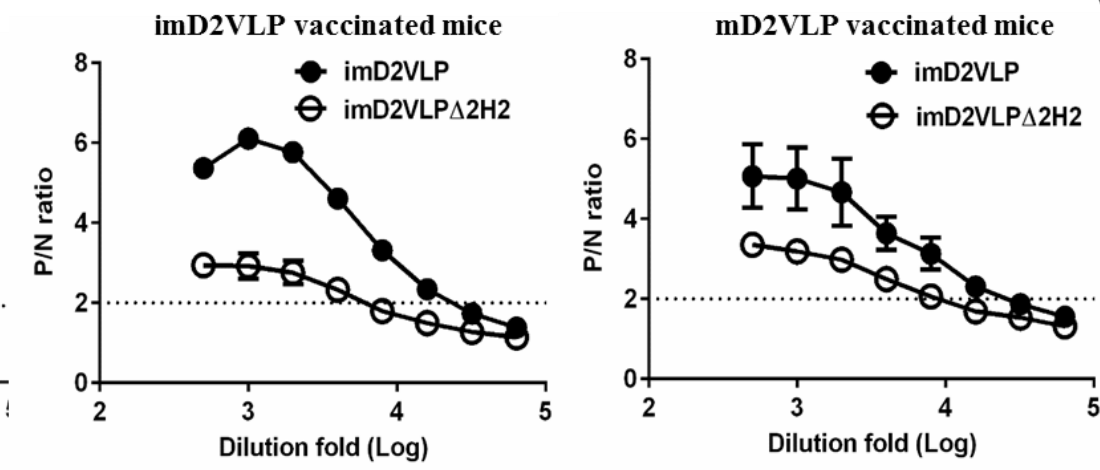

(E)

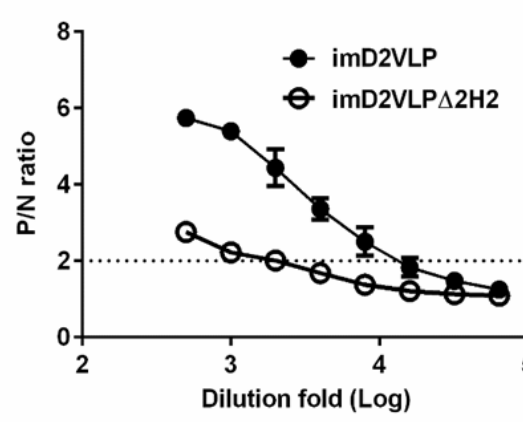


Figure 5. Total antigen-specific IgG, neutralizing titers and proportion of anti-prM antibodies compared among three groups of mice immunized with wtD2VLP, imD2VLP or mD2VLP. (A) D2VLPs were concentrated and purified from clarified supernatants. The total protein concentration of purified imD2VLP and mD2VLP were first determined by the Bradford assay and then subjected to antigen-capture ELISA using 2-fold serial dilutions. The standard curve was used to titrate both antigens as equal amounts for the subsequent assays. (B) The endpoint IgG titer of 12-week post-immunization mouse sera was measured by antigen-capture ELISA, using equal amounts of homologous and heterologous purified D2VLP antigens. All endpoint titers were $\log _{10}$ transformed and depicted as geometric means with $95 \%$ confidence intervals. (C) The neutralizing antibody titers at 50\% antigen focus-reduction micro neutralization (FR $\mu$ NT50) in Vero cells infected with DENV-1 to 4. (D) Binding reactivity of serial dilutions of anti-wtD2VLP (left), anti-imD2VLP (center), and anti-mD2VLP (right) mouse sera were analyzed by ELISA using equal amounts of wild-type (imD2VLP) and mutant imD2VLP $(\triangle 2 \mathrm{H} 2)$ antigens. (E) Proportions of 2H2-like, anti-prM antibodies from two different D2VLP immunization groups were calculated based on the formula $100 *[(\mathrm{OD} 450 \mathrm{imD} 2 \mathrm{VLP}-\mathrm{OD} 450 \Delta 2 \mathrm{H} 2) / \mathrm{OD} 450 \mathrm{imD} 2 \mathrm{VLP}]$ at a $1: 1000$ dilution of mouse sera. All data presented are based on a representative of three independent experiments with two replicates from $\mathrm{n}=5$ mice sera per group per experiment and expressed as mean \pm SEM. The statistical significance was determined using the two-tailed Mann-Whitney $U$ test to account for non-normality of the transformed data. *, $P<0.05 ; * *, P<0.01 ; * * * *, P<0.0001$. 
A
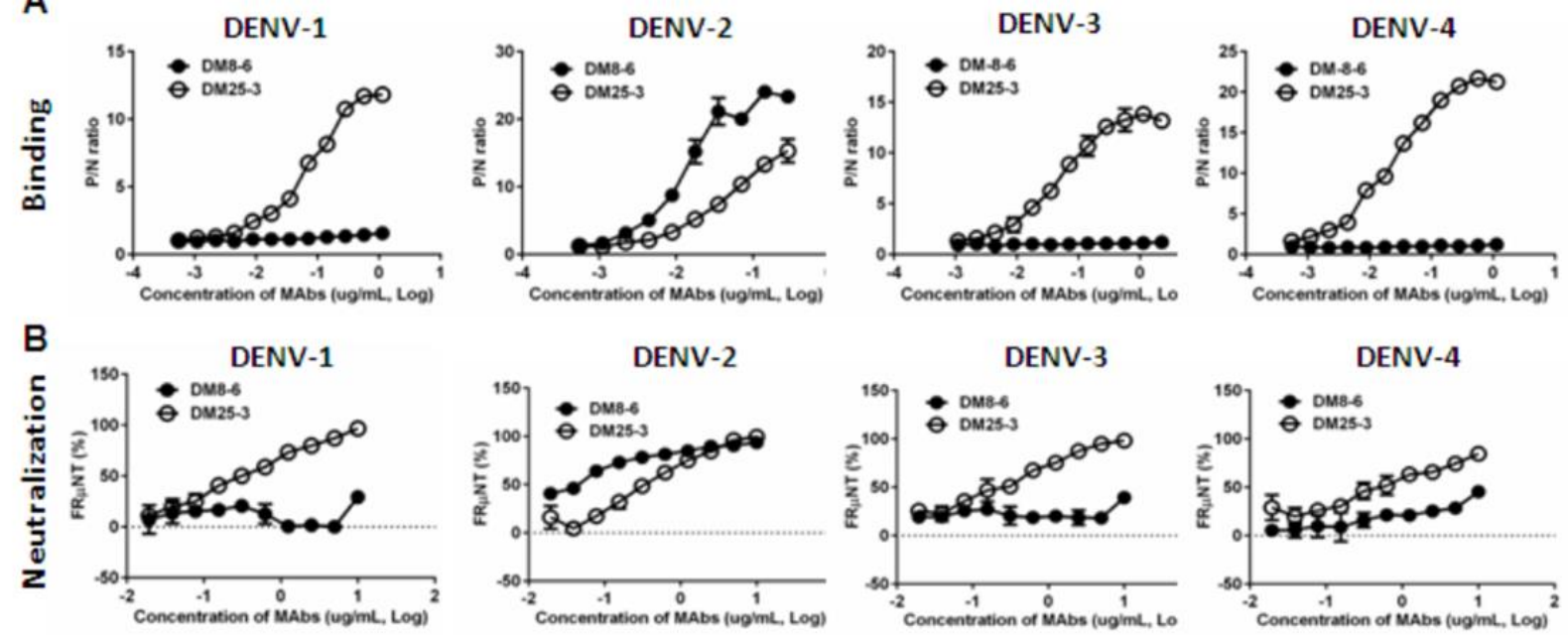

DENV-2

DENV-3
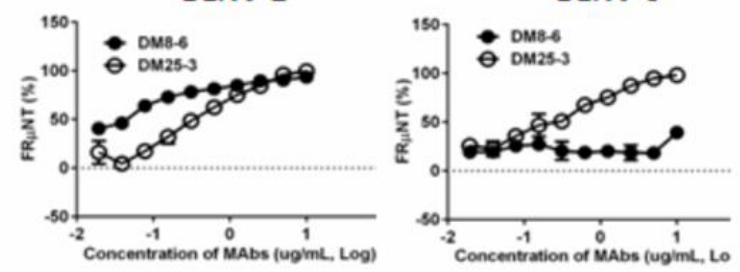

DENV-4

C
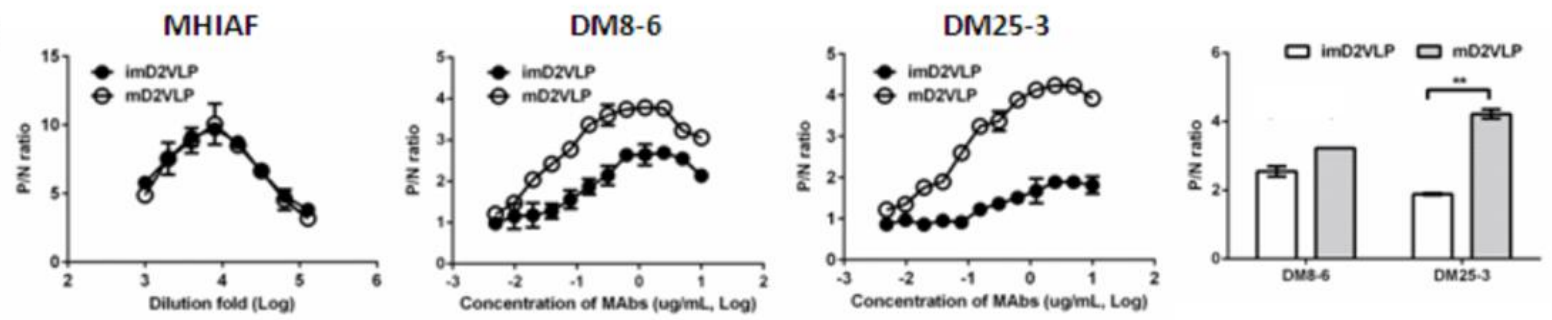

D
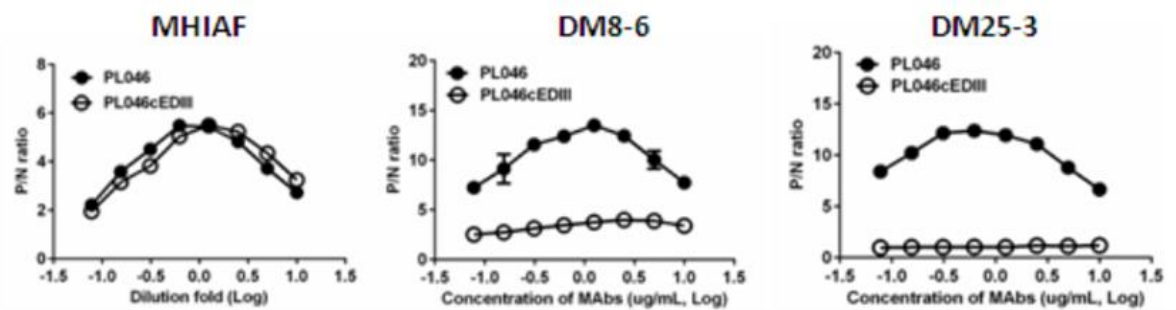

E imD2VLP group sera
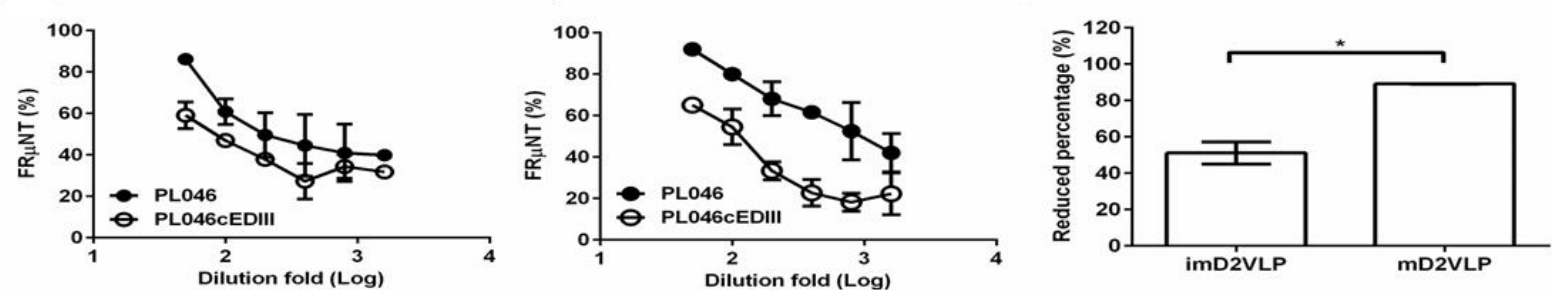

Figure 6. Characterization of murine monoclonal antibodies (MAbs) generated from mouse 
1029 Equal amount of both imD2VLP and mD2VLP was properly titrated by antigen-capture ELISA 1030 using mouse hyper-immune ascitic fluid (MHIAF) against DENV-2 (left). The difference in

1031 binding activities of both MAbs is presented by a bar graph (right). (D) A recombinant DENV-2 1032 virus was produced by replacing domain III with a consensus sequence of domain III 1033 (PL046cEDIII) ${ }^{31}$ and the binding activity of DM8-6 (center) and DM25-3 (right) was compared 1034 with that of parental DENV-2 strain PL046. Equal amount of both PL046 and PL046cEDIII was 1035 properly titrated by antigen-capture ELISA using mouse hyper-immune ascitic fluid (MHIAF) 1036 against DENV-2 (left). (E) FR $\mu$ NT of two-fold diluted mice sera immunized with mD2VLP and 1037 imD2VLP against parental PL046 and PL046cEDIII DENV-2 viruses ( $\mathrm{n}=5$ per group per 1038 experiment). The differences in FR $\mu$ NT50 from mD2VLP and imD2VLP immunization groups 1039 were converted to bar chart at 1:1000 fold dilution of mice sera. The conversion was based on 1040 the formula $100 *[\mathrm{FR} \mu \mathrm{NT} 50$ of (PL046- PL046cEDIII)/ FR $\mu$ NT50 of PL046]. P/N ratio refer to 1041 the antibody binding magnitude between designated VLP-containing (P) and VLP-free culture 1042 supernatant $(\mathrm{N})$ by dividing the absorbance of $\mathrm{P}$ by that of $\mathrm{N}$. The data are presented as means $\underline{ \pm}$ 1043 SEM from three independent experiments with two replicates. The two-tailed Mann-Whitney $U$ 1044 test was used to test statistical significance. ${ }^{*}, P<0.05$. **,$P<0.01$. 


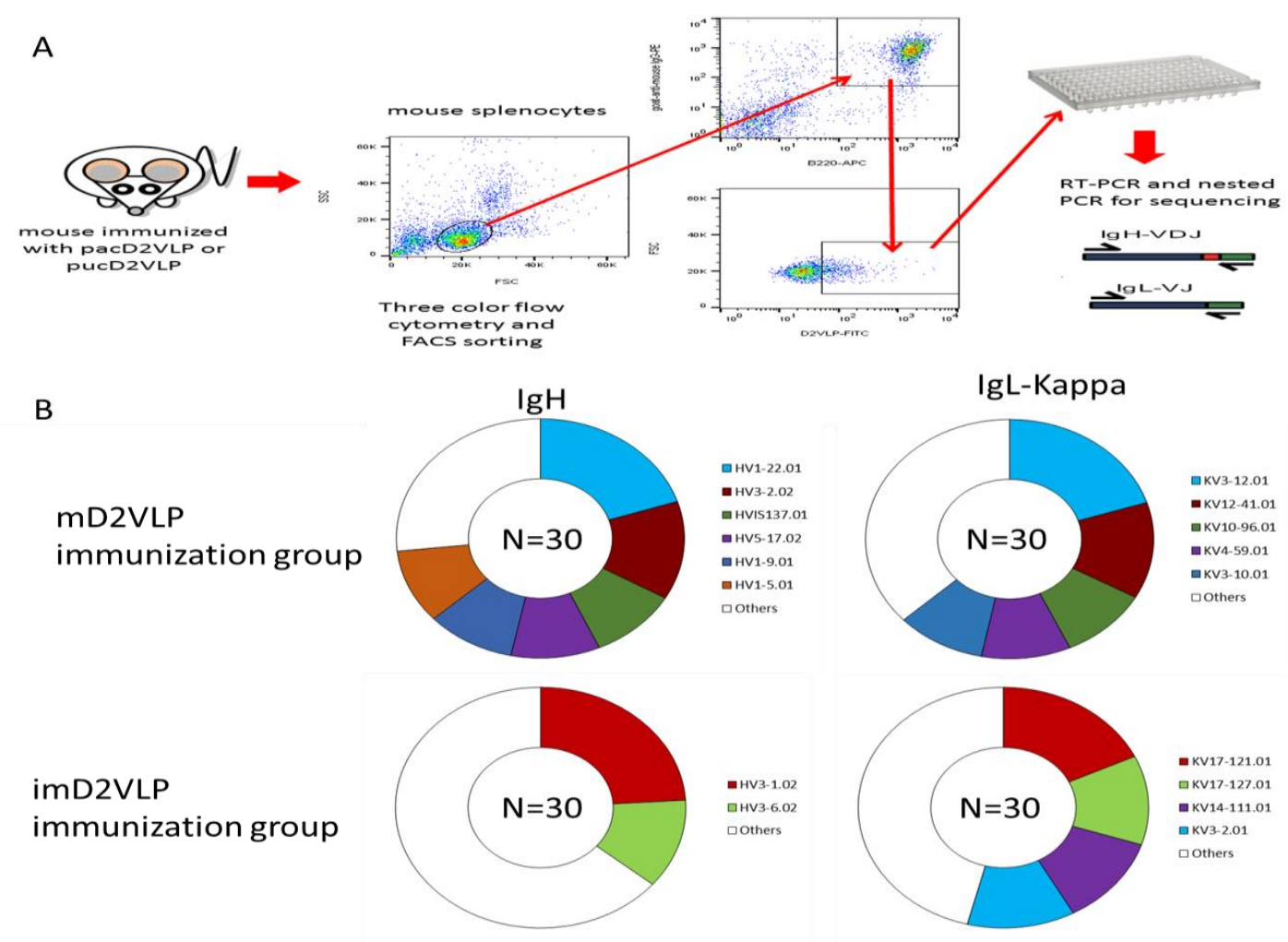

Figure 7. Overview of the experimental design and DENV-specific B-cell repertoires from 
A

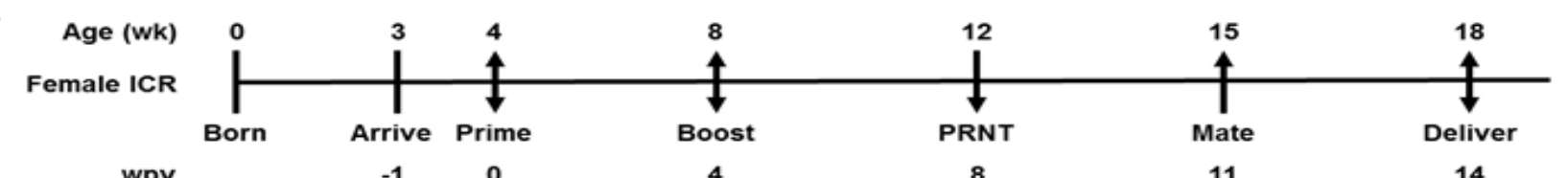

wpv

$-10$

B

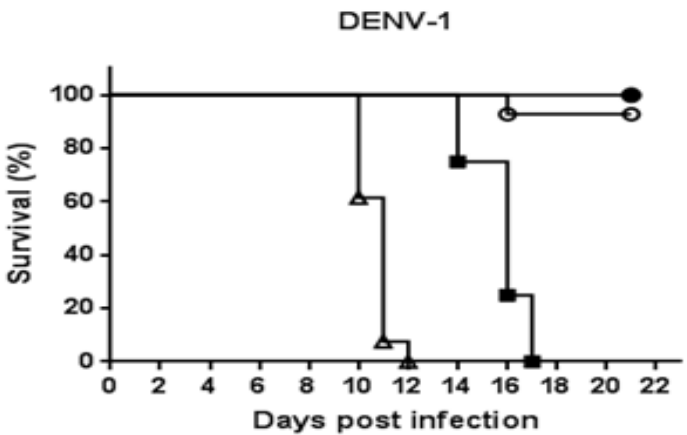

DENV-3

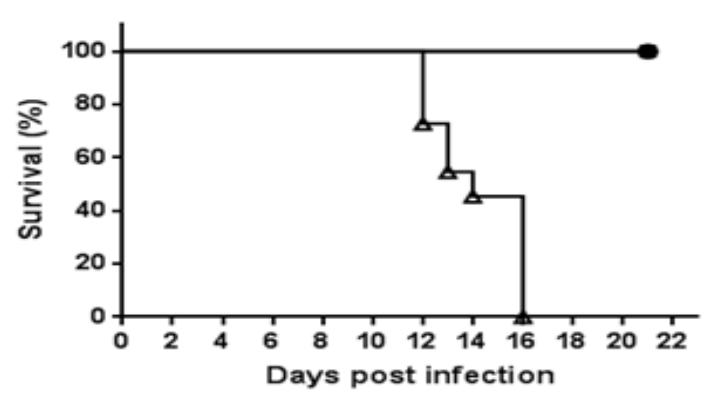

- $w t D 2 V L P(n=8)$

- imD2VLP(n=12)

- mD2VLP(n=14)

$\triangle \mathrm{TNE}(\mathrm{n}=13)$

- wtD2VLP(n=4)

- imD2VLP(n=9)

- mD2VLP $(n=10)$

$\star \mathrm{TNE}(\mathrm{n}=11)$
ICR pups

Challenge

day $0 \quad 2$

DENV-2

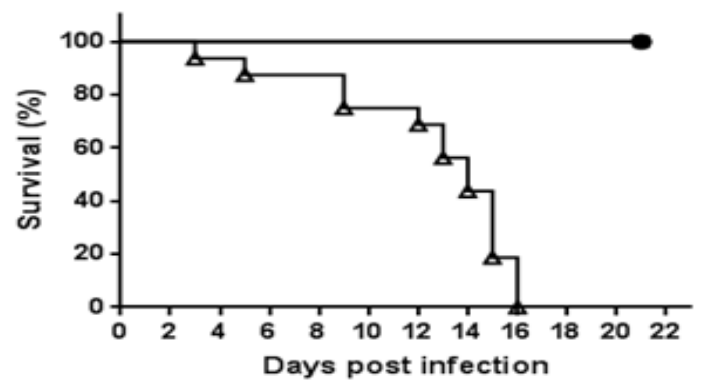

DENV-4

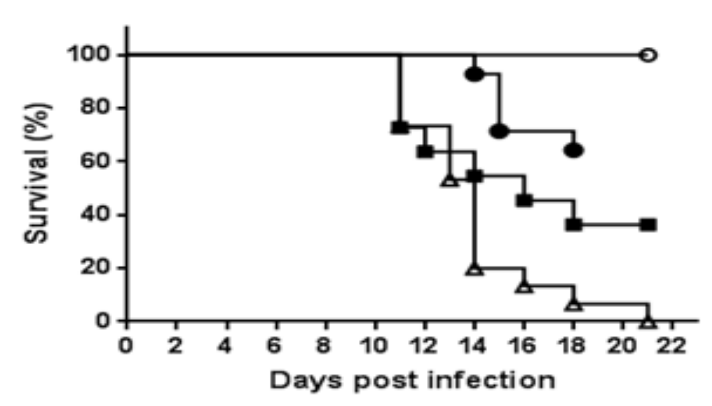

- wtD2VLP $(n=12)$

- imD2VLP $(n=14)$

- mD2VLP $(n=13)$

$\star \mathrm{TNE}(\mathrm{n}=16)$
- wtD2VLP(n=11)

- imD2VLP $(n=14)$

- mD2VLP $(n=8)$

$₫ \mathrm{TNE}(\mathrm{n}=14)$ 
1061 four 4-week-old, female, ICR mice were injected intramuscularly with imD2VLP and mD2VLP at week post vaccination (wpv) 0 and 4 at a dose of $4 \mu \mathrm{g} / 100 \mu \mathrm{L}$. Mice were bled from the retro-orbital sinus at week 4 following the second injection, and individual mouse serum collected from immunized females 1 week prior to mating was evaluated for the presence of the total IgG titer and the virus neutralization response by ELISA and focus-forming micro-neutralizing assay (FRNT). For the evaluation of passive protection by maternal antibody, ICR pups from the mating of non-immunized males with immunized females 11 weeks post initial vaccination were obtained for viral challenge. Pups from unvaccinated females were used as the challenge control. ICR pups from the designated groups were challenged individually through intracranial route at 2 days after birth with $10^{4}$ focus-forming unit (FFU) which were equivalent 141, 61, 11, 1000 times of 50\% lethal doses (LD50) of DENV-1, to DENV-4, respectively. The percent survival of the mice was evaluated daily for up to 21 days. (B) Survival curve of pups delivered from the female mice receiving mD2VLP, imD2VLP monovalent vaccine or TNE control, then challenge with DENV-1 to 4 after birth. The in vivo protective efficacy of DENV-2 1071 monovalent vaccine is maturity-dependent. $\mathrm{N}$ in parentheses indicated the numbers of pups of each group. Kaplan-Meier survival curves were analyzed by the log-rank test. ***, $P<0.001$.

1073 


\begin{tabular}{|c|c|c|c|}
\hline Name & Primer sequence (5'-3') & $\begin{array}{l}\text { Amino acid } \\
\text { substitution }\end{array}$ & Secreted \\
\hline \multirow[t]{3}{*}{$\mathrm{mD} 2 \mathrm{VLP}$} & 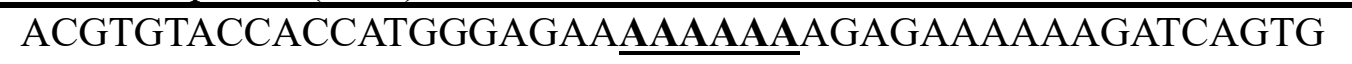 & His-Lys, Arg-Lys & $\mathrm{Y}$ \\
\hline & TGGGACGTGTACCACCATGGTAGTAAAAAAAAGAGAAAAAAGATC & Gly-Val, Glu-Val & Y \\
\hline & CATGGTAGTAAAAAAAAGATCAAAAAGATCAGTGGCACTCG & Glu-Ser & $\mathrm{Y}$ \\
\hline imD2VLP & AGAACATAGAAGAGAATCAACATCAGTGGCACTCG & Lys-Ser, Arg-Thr & $\mathrm{Y}$ \\
\hline \multirow[t]{3}{*}{$\triangle 2 \mathrm{H} 2$} & AGGGAAAAGTCTTCTGTTTCCAACAGAGGATGGCGTGAAC & Lys-Pro & $\mathrm{Y}$ \\
\hline & CAGCAGACAAGAGAAAGGG $\underline{\mathbf{G A}} \mathbf{C A G T C T T C T G T T T C C A A C A G ~}$ & Lys-Asp & $\mathrm{Y}$ \\
\hline & CATAGCTTGTGCAGGCGCC $\underline{\mathbf{G C C}}$ ATTTAACCACACGTAAC & Phe-Ala & $\mathrm{Y}$ \\
\hline W101G & TCCATGGTAGACAGAGGAGGGGGAAATGGATGTGGACTA & Trp-Gly & Y \\
\hline N103K & GACAGAGGATGGGGAAAAGGATGTGGACTATTTGGA & Asn-Lys & $\mathrm{N}$ \\
\hline G104Q & AGACAGAGGATGGGGAAATCAATGTGGACTATTTGGAAAGG & Gly-Gln & $\mathrm{N}$ \\
\hline K307E & TCTATGTGCACAGGAAAGTTTGAAGTTGTGAAGGAAATAGCAGAA & Lys-Glu & $\mathrm{N}$ \\
\hline $\mathrm{K} 310 \mathrm{E}$ & 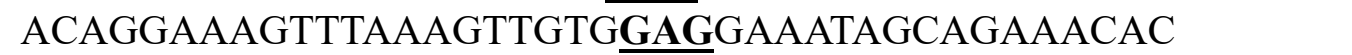 & Lys-Glu & $\mathrm{Y}$ \\
\hline E311R & CAGGAAAGTTTAAAGTTGTGAAGCGAATAGCAGAAACACAACATGG & Glu-Arg & $\mathrm{Y}$ \\
\hline E314R & AAAGTTGTGAAGGAAATAGCACGAACACAACATGGAACAATAGTT & Glu-Arg & $\mathrm{N}$ \\
\hline $\mathrm{T} 315 \mathrm{H}$ & TTGTGAAGGAAATAGCAGAAC $\overline{\text { ACCAACATGGAACAATAGTTAT }}$ & Thr-His & $\mathrm{N}$ \\
\hline Q316P & GTGAAGGAAATAGCAGAAACACCACATGGAACAATAGTTATCAGA & Gln-Pro & Y \\
\hline $\mathrm{H} 317 \mathrm{E}$ & AAGGAAATAGCAGAAACACAA $\overline{\overline{\mathbf{G A A}}}$ GGAACAATAGTTATCAGAGTG & His-Glu & Y \\
\hline P364R & GACAGAAAAAGATAGCCGGGTCAACATAGAAGCAGAACCT & Pro-Arg & $\mathrm{N}$ \\
\hline
\end{tabular}


W391G

F392A

1076

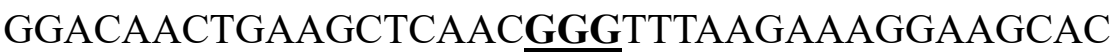
ACAACTGAAGCTCAACTGGGCTAAGAAAGGAAGCACGCTG
Trp-Gly

Phe-Ala
$\mathrm{N}$

$\mathrm{Y}$

1077 
1078

1079 
(A)

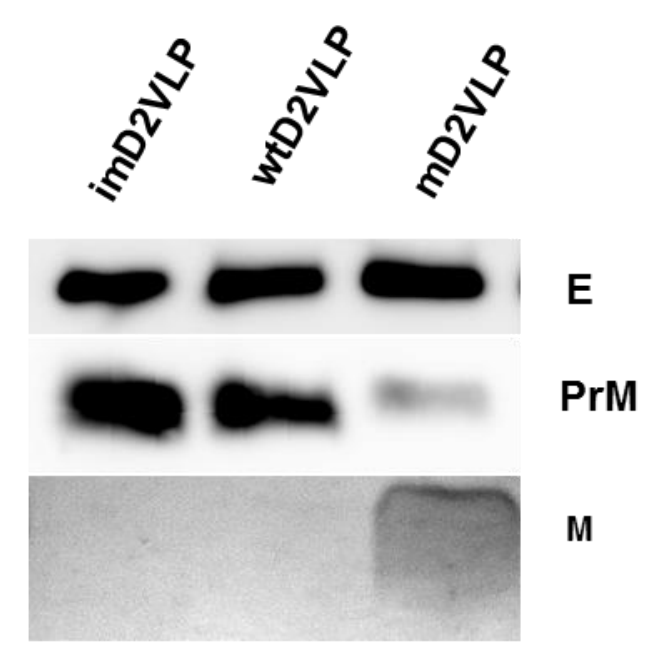

(B)

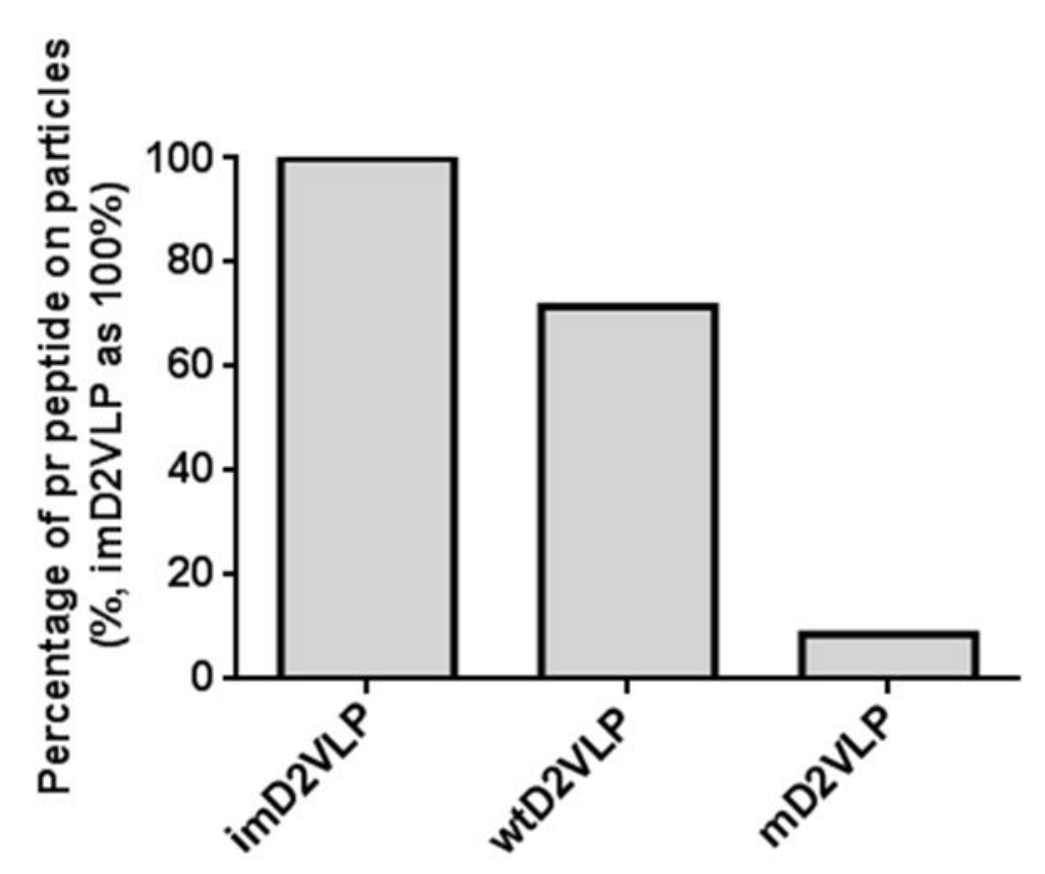

1082 Figure S1. Comparison of prM cleavage among different DENV-2 virus-like particles (D2VLP). (A) Culture supernatants of mD2VLP and imD2VLPs were

1083 collected and purified after electroporation with the respective plasmids. Five micrograms of proteins were loaded onto a 12\% non-reducing Tricine-SDS-PAGE.

1084 E, prM and M proteins were assayed by Western blot using mouse hyper-immune ascitic fluids (MHIAF, 1:2000), MAb 2H2 (0.5 $\mu \mathrm{g} / \mathrm{mL})$ and anti-M protein 

prM band by Bio-1D software.

1088 
(A)

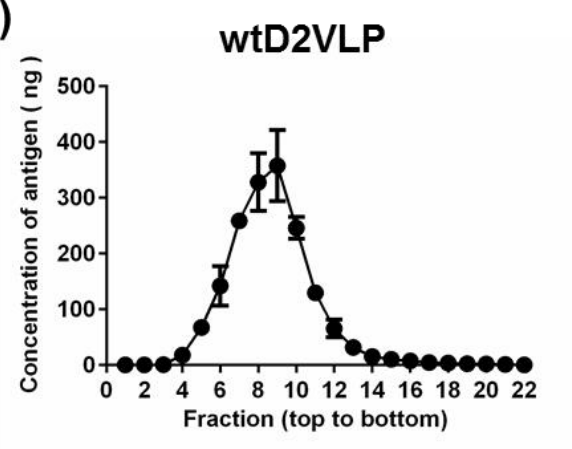

(B)

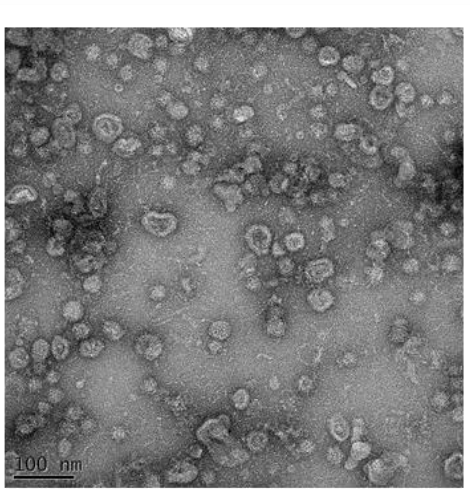

(C)

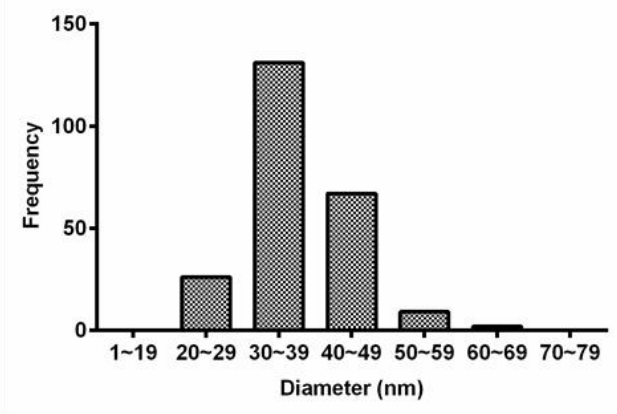

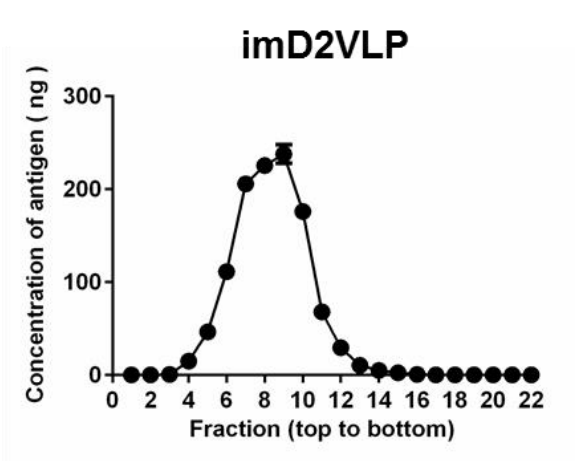
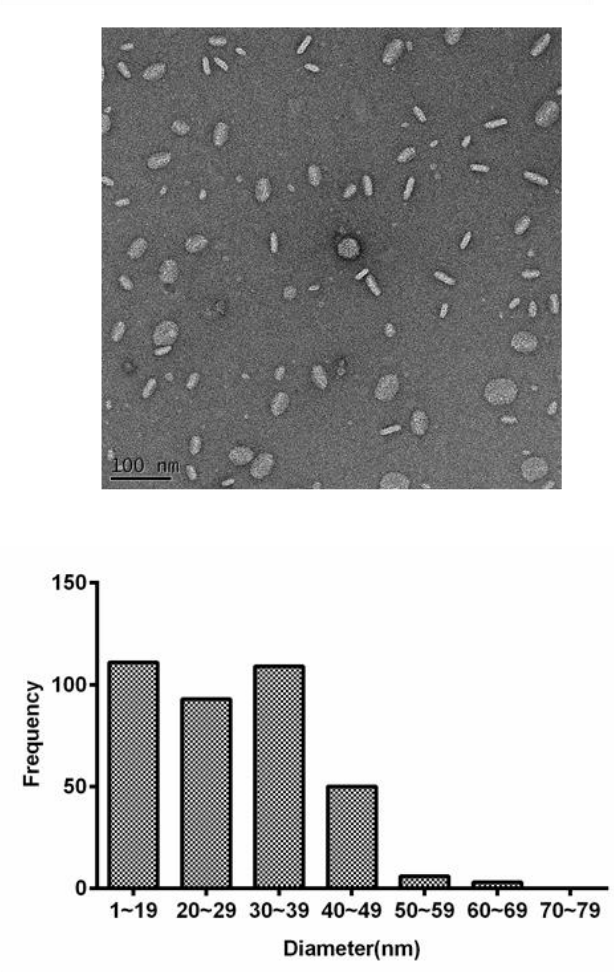
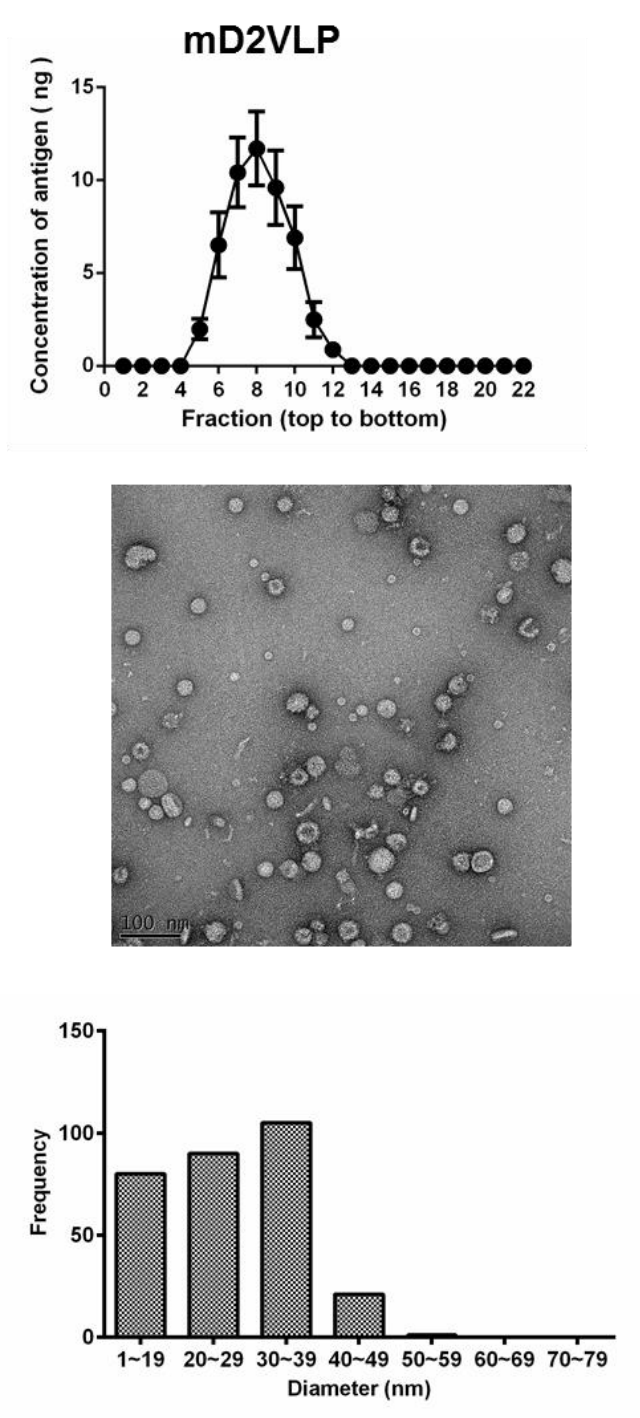
(D)

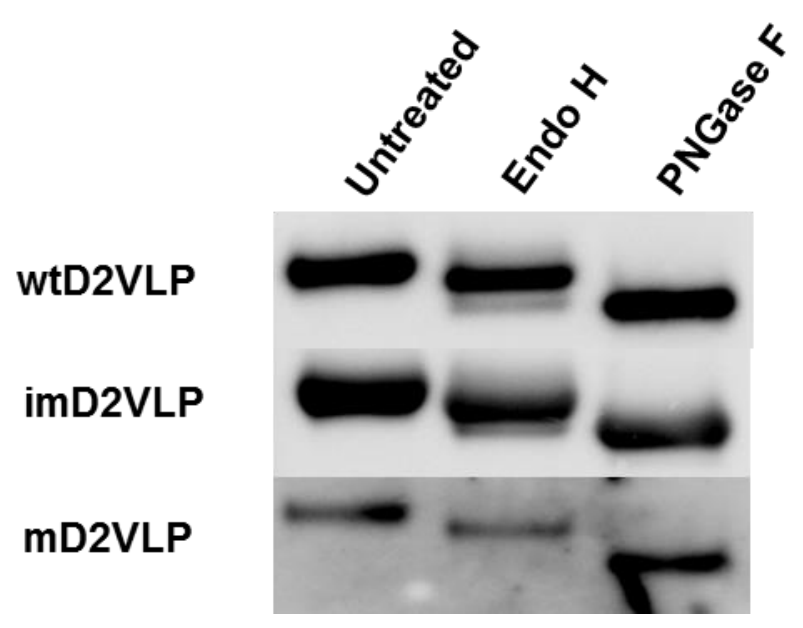

1092

1093 Figure S2. Comparison of physical properties of D2VLP among different DENV-2 virus-like particles (D2VLP). (A) Equilibrium banding profiles 1094 of wtD2VLP (left), imD2VLP (center) and mD2VLP (right) after rate-zonal centrifugation on a 5-25\% linear sucrose density gradient at

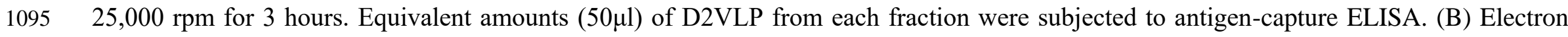
1096 micrographs at 50,000-fold magnification of wtD2VLP (left), imD2VLP (center) and mD2VLP (right) stained with uranyl acetate from the 1097 peak fraction in (A). (C) The diameter of 200 randomly selected particles of wtD2VLP (left), imD2VLP (center) and mD2VLP (right) from 1098 representative electron micrographs as in (B) were determined by software Gatan Digital Micrograph. (D) Endoglycosidase treatment of 


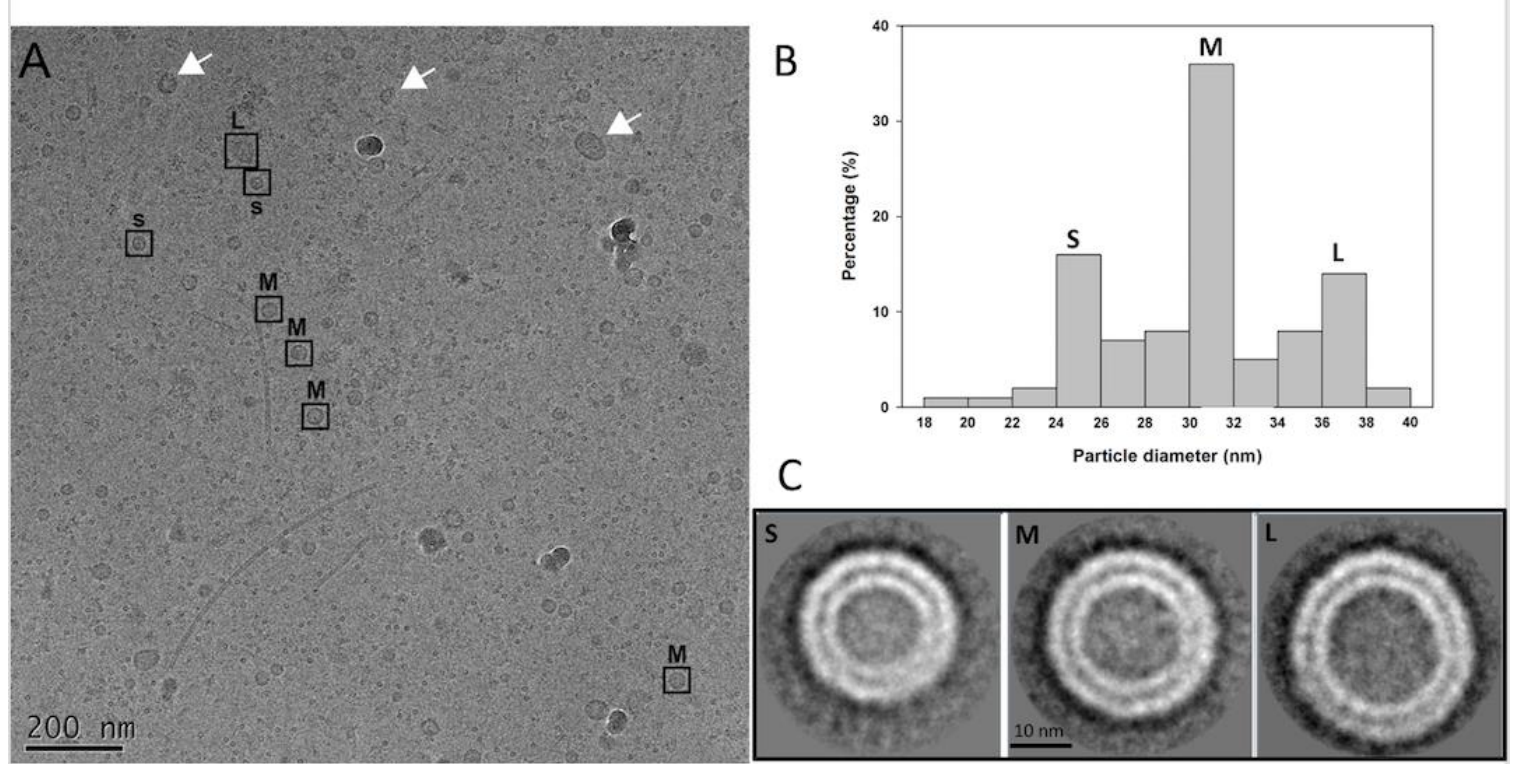

1103

1104 Figure S3. The cryo-EM images and 2D analysis of the particles. (A) Cryo-EM images of purified mD2VLP showed spherical particles (boxed) among irregular

1105 or incomplete structures (arrows). Irregularly-shaped particles were eliminated through visual inspection, while the spherical particles were subjected to further

1106 image analyses. (B) The particle size analyses showed the size variation in the sample and that the major peaks were located at $\sim 26 \mathrm{~nm}, \sim 31 \mathrm{~nm}$ and $\sim 36 \mathrm{~nm}$

1107 diameter size classes which were therefore denoted by small ('S'), medium ('M') and large ('L'). Each of the 'S', 'M' and ' $L$ ' particles identified was labeled.

1108 (C) The 2D image analyses showed that the particles had two distinct layers and that the particles classes with diameters of $\sim 31 \mathrm{~nm}$ had more solid features than 1109 others.

1110 


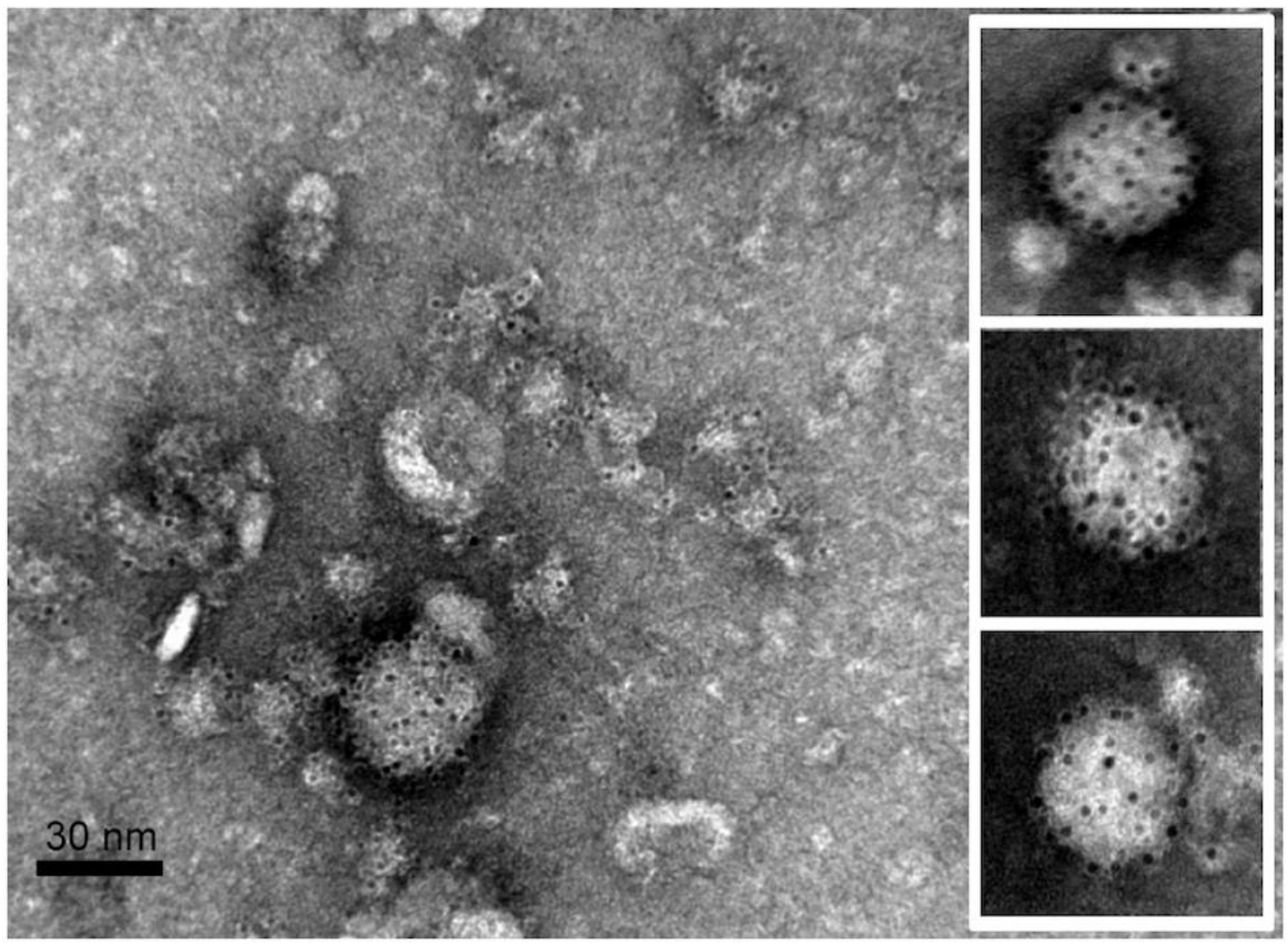

1111

1112 Figure S4. The EM images of immunogold-labeled mD2VLPs. The E proteins were labeled with domain III-specific monoclonal antibody (MAb 32-6), and 1113 conjugated with 6-nm gold particles.

1114 

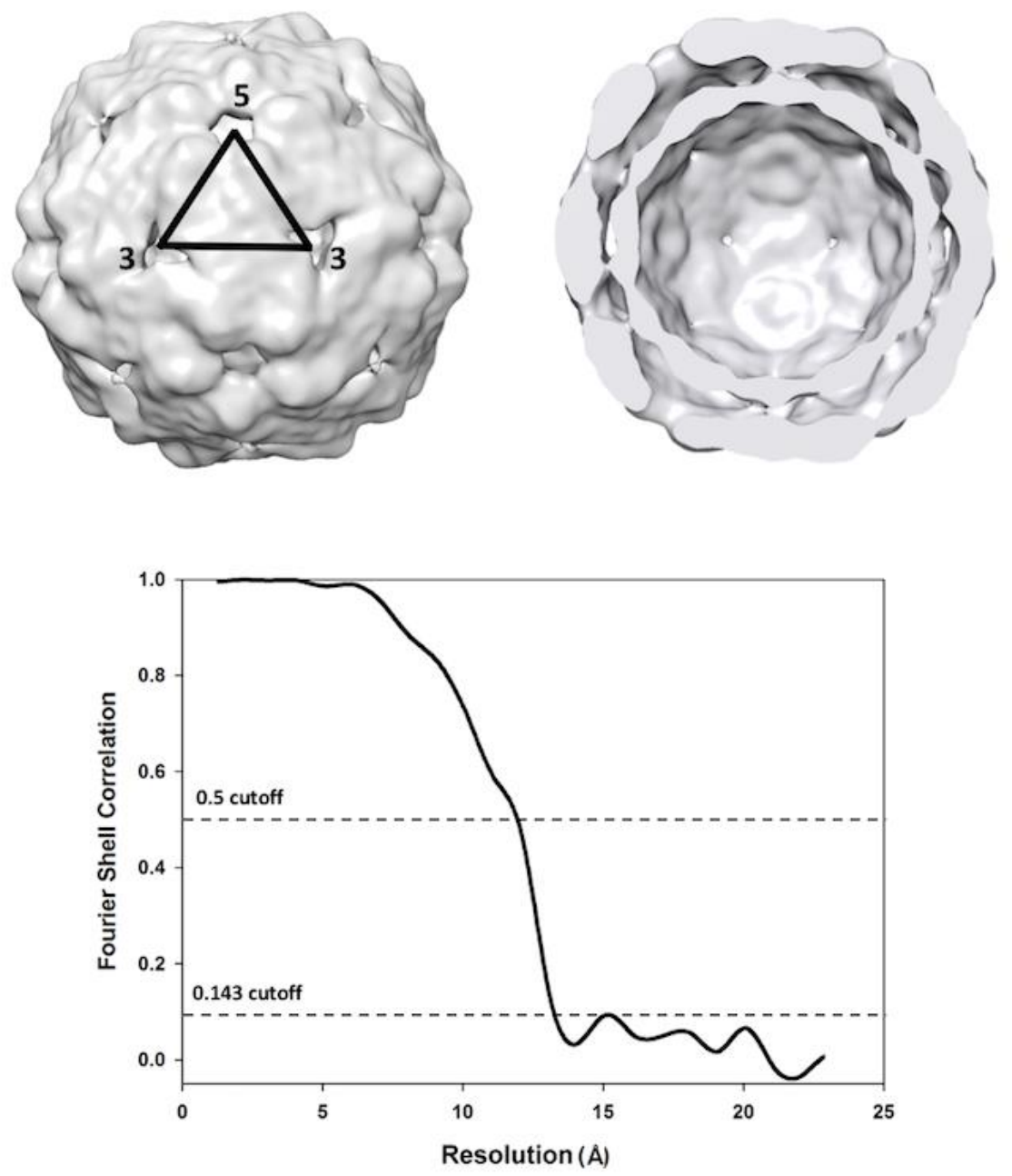

1117

Resolution (A)

1118

1119 Figure S5. The cryo-EM structure of m2DVLP. The reconstructed cryo-EM map of the

$1120 \mathrm{mD} 2 \mathrm{VLPs}$ was shown on the left. An asymmetric unit was indicated. On the right, the closest

1121 half of the density map has been removed to reveal the hollow structure. The resolution of the

1122 final reconstruction was determined to be $13.1 \AA$ using a gold standard resolution estimate at 1123 cutoff 0.143 (lower panel). 

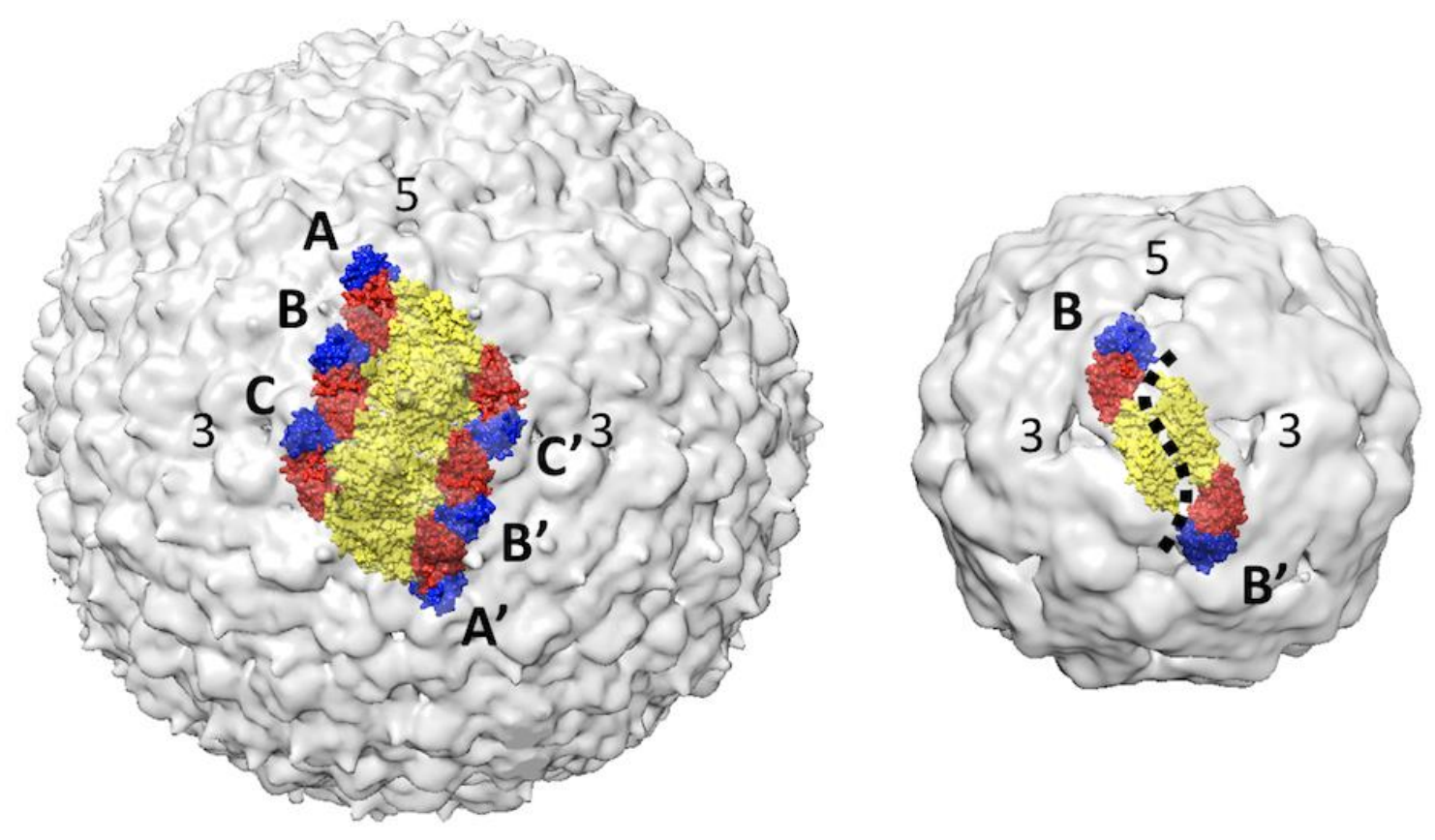

1127 Figure S6. The E protein rafts in a virion (left) and mD2VLP (right). The E protein forming

1128 the rafts in the virion were shown in the left panel where the three individual E proteins in the

1129 asymmetric unit were labeled A, B, and C and the E proteins in the neighboring asymmetric

1130 unit are labeled A', B', and C'. The icosahedral 2-fold E protein dimers (B and B') of

$1131 \mathrm{mD} 2$ VLP moved apart from each other causing the groove (dashed line, right). 

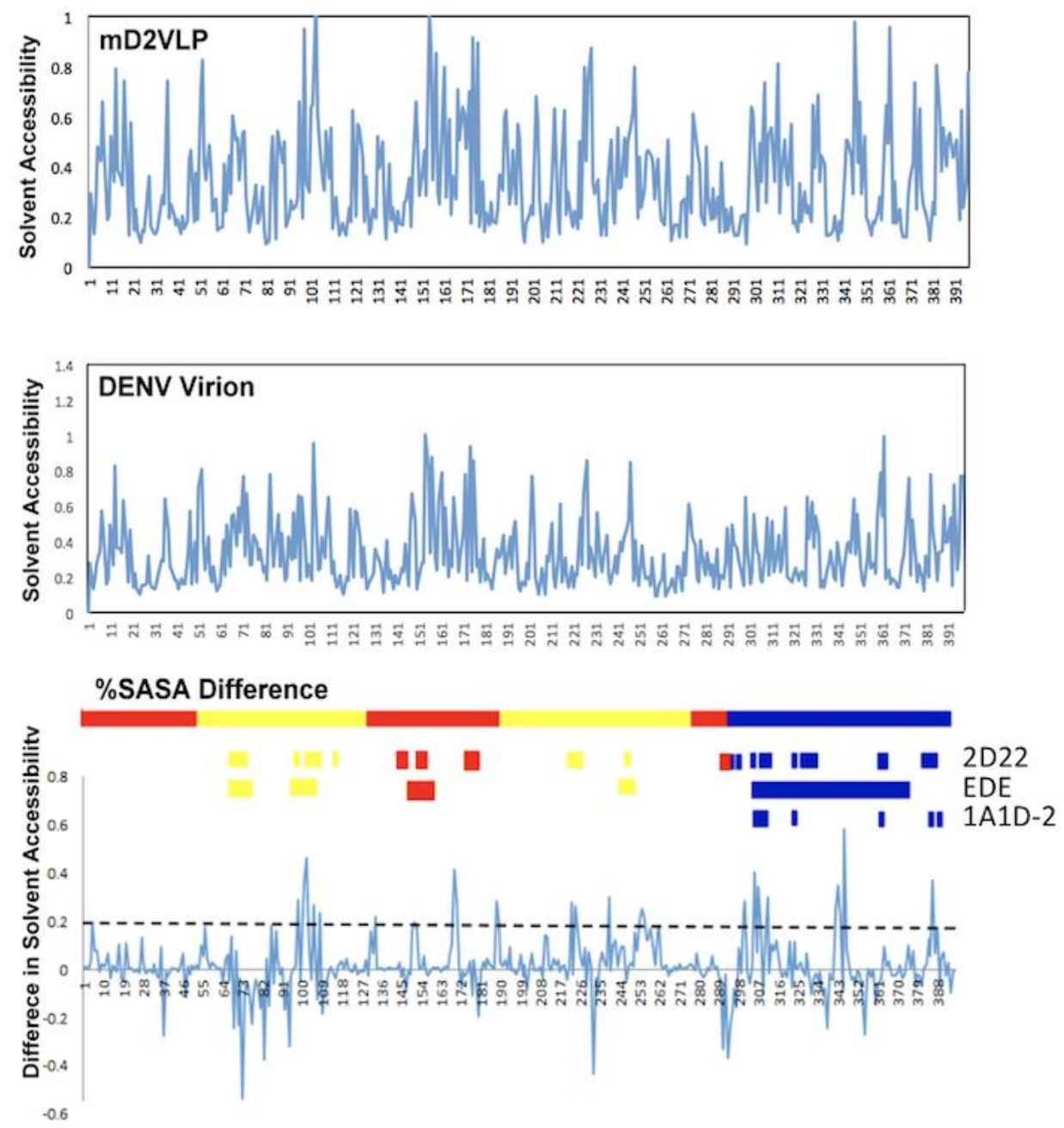

1137

1138 Figure S7. The solvent accessibility analyses. Solvent accessibility of the dengue particle

1139 soluble envelope ( $\mathrm{sE}$ ) protein amino acid (aa) residues 1-396 in DENV virion and mD2VLP

1140 (middle and top, respectively). The all-atom model of VLP was built using MODELLER ${ }^{18}$,

1141 and the all-atom model virion was built based on the cryo-EM structure of the mature dengue

1142 virus at $3.5 \AA$ resolution (PDB ID: 3 J27). The relative solvent-accessible surface area (SASA)

1143 of both models was calculated using the POPS program. The plot shown represents the 
1144 difference in relative solvent-accessible surface area ( $\Delta \%$ SASA) (Bottom). A positive value

1145 of \%SASA means the residue becomes more exposed in mD2VLP than in DENV VLP.

1146 Domains I, II and III are highlighted in red, yellow and blue, respectively. The residues

1147 interacting with antibodies 2D22, EDE and 1A1D-2 are shown.

1148

1149 

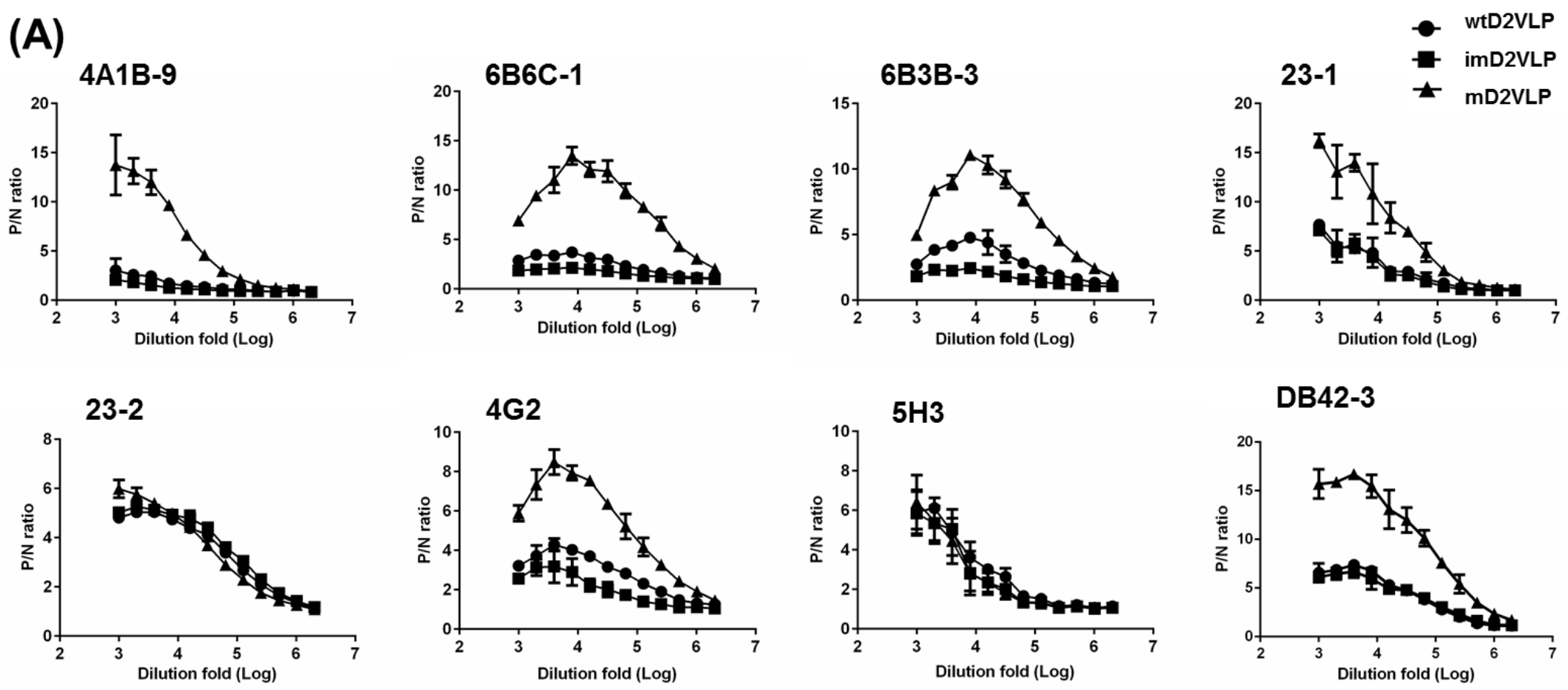

\section{DB42-3}

(B)
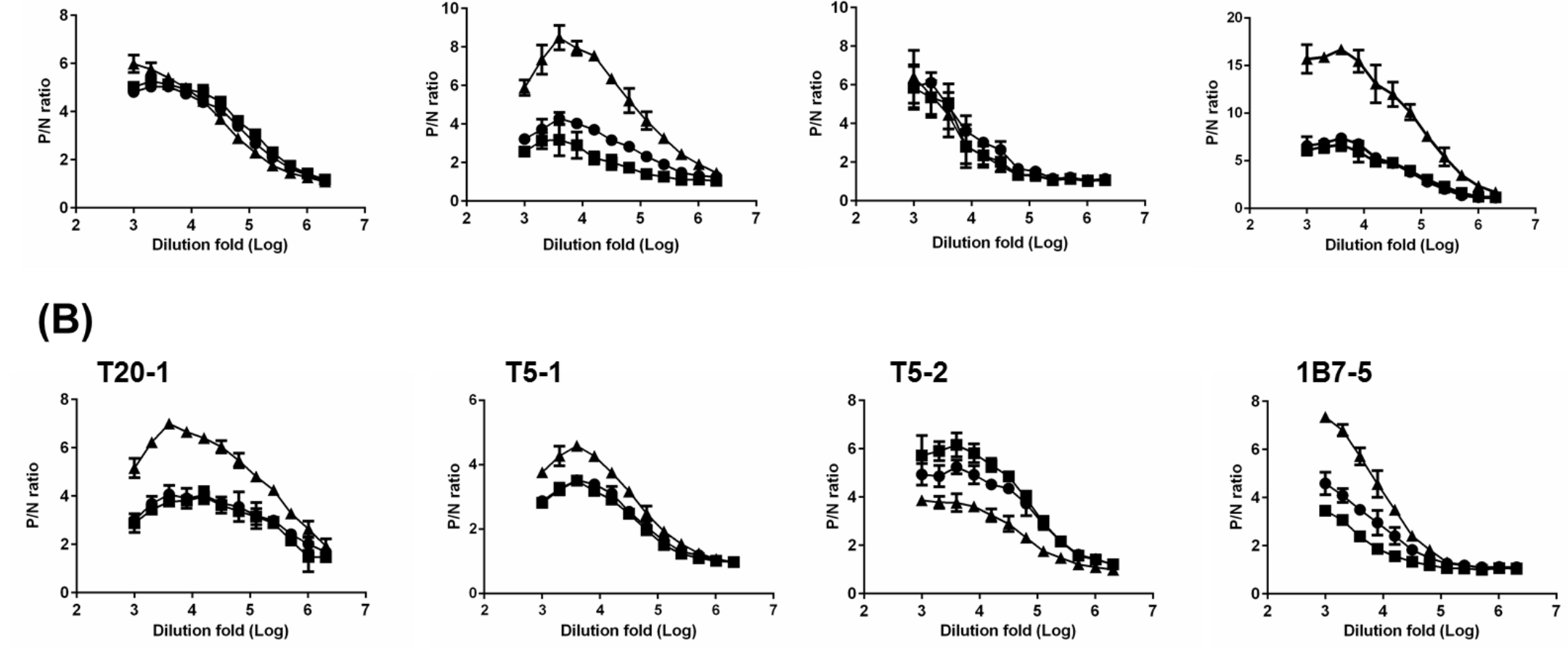
(C) ${ }_{D B 13-19}$

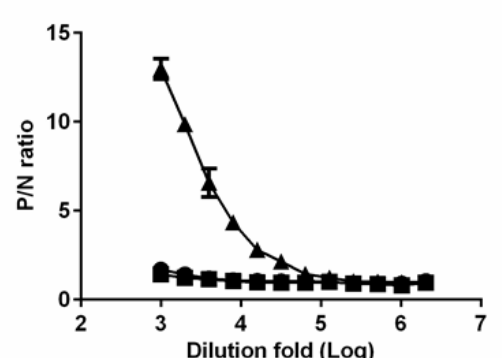

(E)

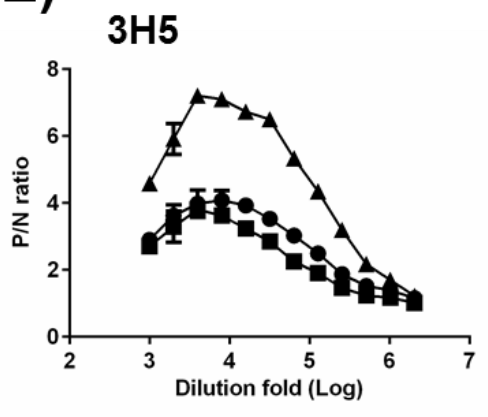

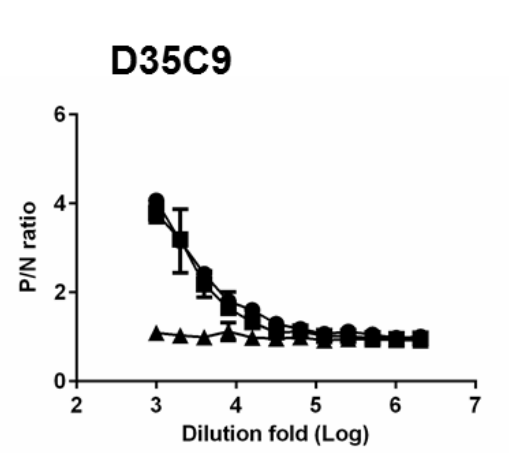

(D)
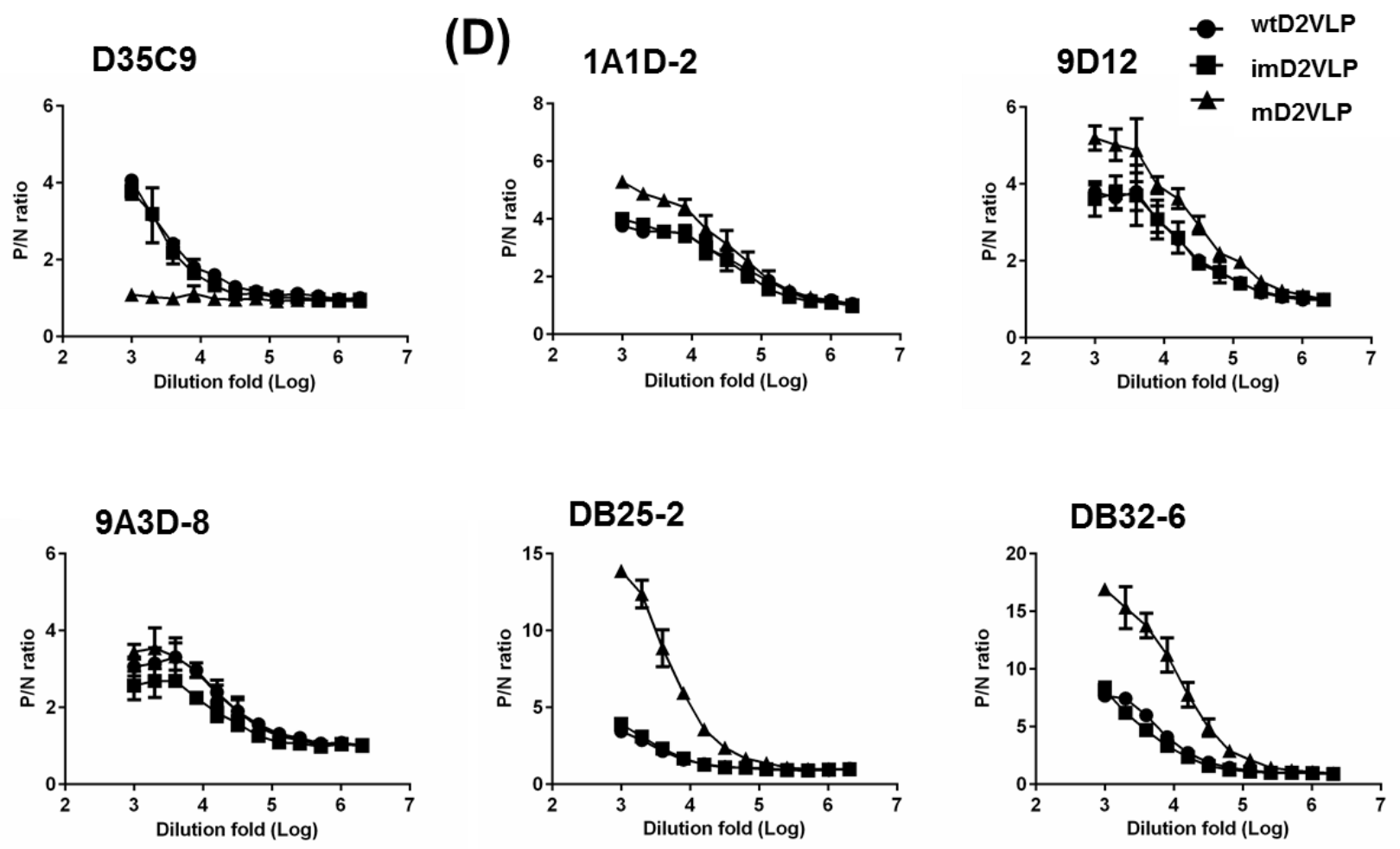

Fig S8. Binding activity curves of a panel of anti-E monoclonal antibodies (Mabs) to three types of D2VLP with different percentage of pr-peptide on particle surface. Binding curves for (A) eight group-reactive Mabs, (B) four subgroup-reactive Mabs, (C, D) four complex- or sub-complex-reactive Mabs, (E) four 


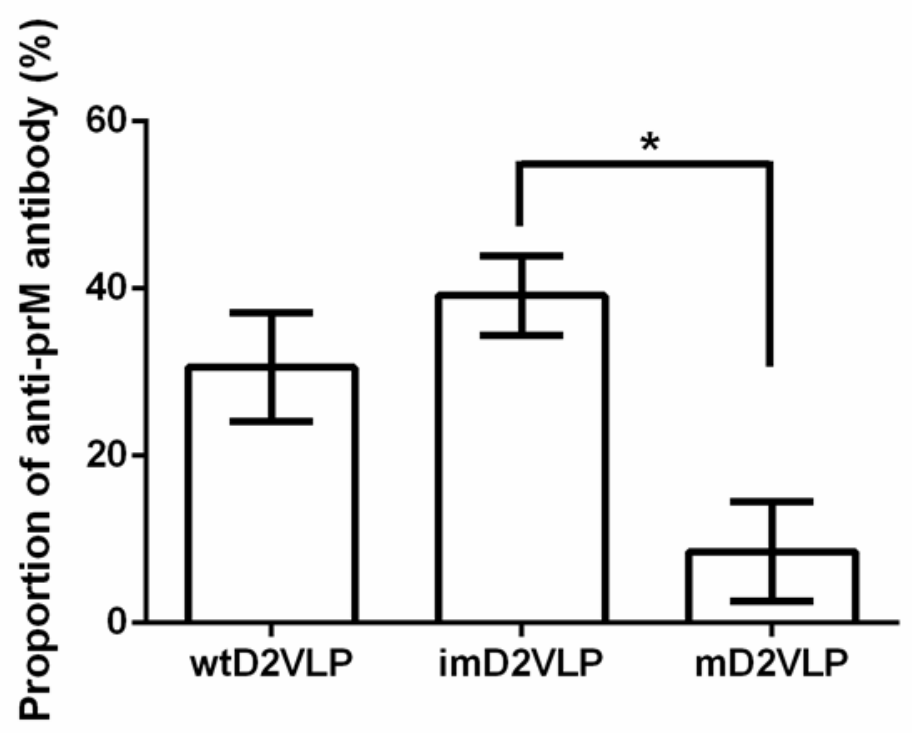

1160

1161

1162 Fig S9. Proportions of anti-prM antibodies from both D2VLP immunization groups were measured using an epitope-blocking ELISA. Percent blocking of HRP-

1163 labeled anti-prM MAb (2H2-HRP) by sera from mice vaccinated with imD2VLP or mD2VLP was determined by the formula 100*[(OD450imD2VLP-

1164 OD450imD2VLP blocked by MAb 2H2)/OD450imD2VLP] using a 1:1000 dilution of mouse sera.

1165 
$\mathrm{Mab} 2 \mathrm{H} 2$

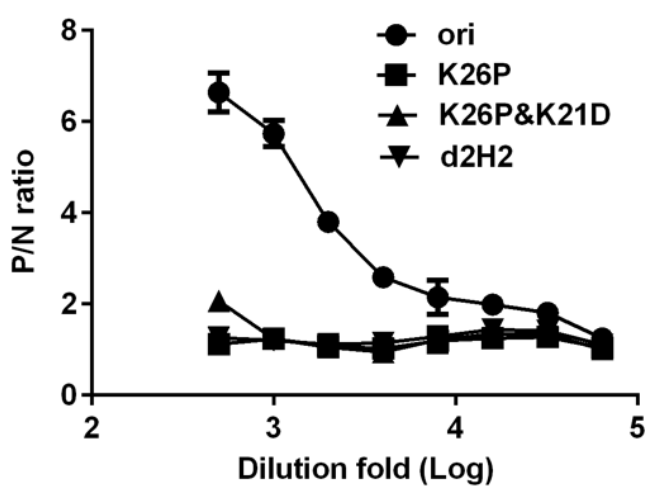

Mab 155-49

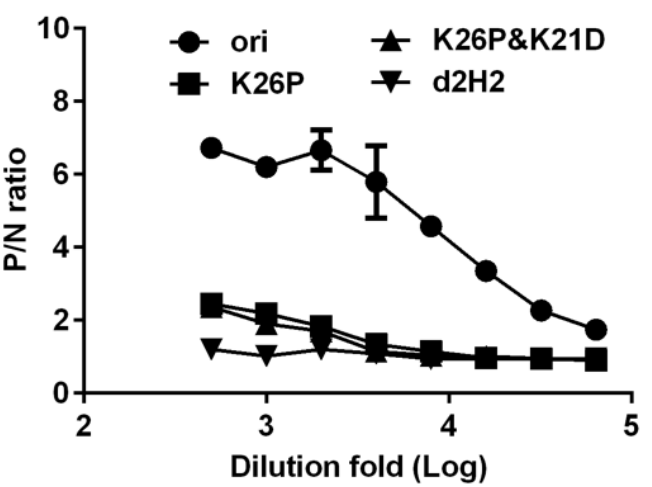

1168

1169 Figure S10. Epitope identification of MAbs 2H2 and 155-49. Single (K26P), double (K26P\&K21D) and triple (K26P\&K21D\&F1A) mutations were performed on imD2VLP-expression plasmids by site-directed mutagenesis. Various D2VLP mutants were expressed in

1171 COS-1 cells by electroporation with plasmid DNAs as indicated and tissue culture media were clarified 3 days after transfection for antigencapture ELISA. Substitutions of K26P on imD2VLP led to a significantly reduced binding activity of MAb 2H2; however, a weak binding can still be detected for MAb 155-49. Double mutation didn't affect the binding of MAb 155-49 on imD2VLP, compared to a single mutation. Only the triple mutation completely abolished the binding of MAb 155-49 on imD2VLP. The antigens used were standardized at a 
$2 \mathrm{H} 2$

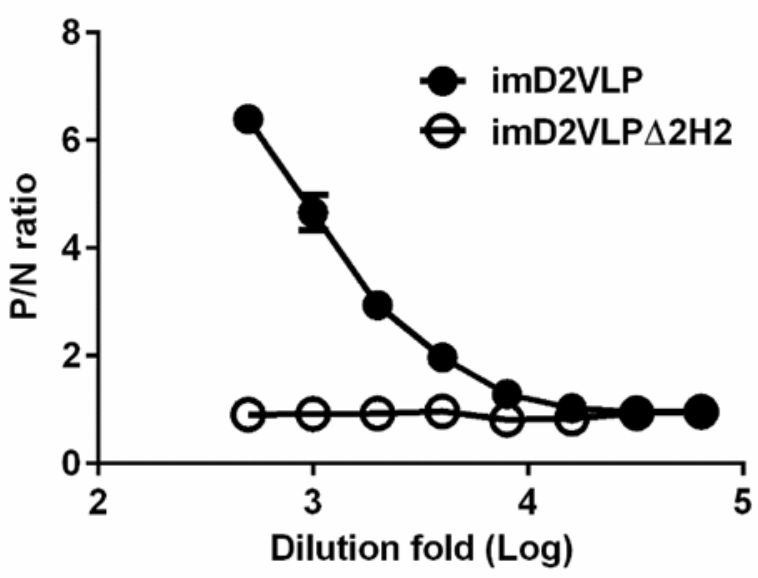

$3 \mathrm{H} 5$

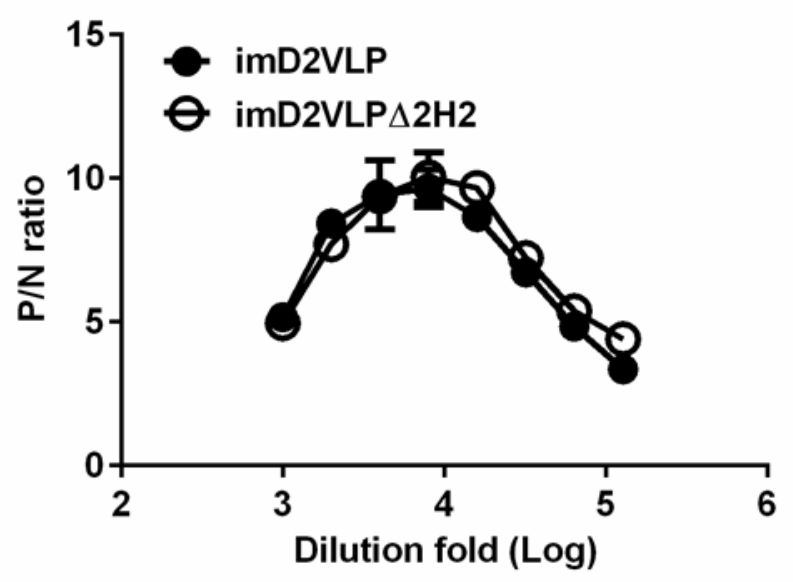

1182 Fig S11. Serial dilutions of imD2VLP and mutant imD2VLP $(\Delta 2 \mathrm{H} 2)$, containing mutations at the MAb 2H2 binding site (F1A, K21D, K26P), were tested for

1183 binding with MAb 2H2 (left) and control antibody 3H5 (right) by ELISA. P/N ratios refer to the antibody binding magnitude between designated VLP-containing

1184 (P) and VLP-free culture supernatant $(\mathrm{N})$ by dividing the absorbance of $\mathrm{P}$ by that of $\mathrm{N}$. 


\section{A}

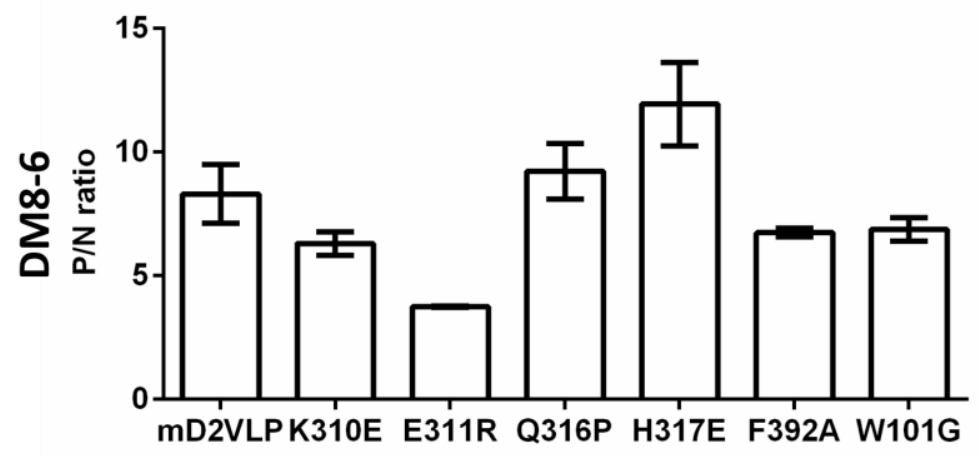

B

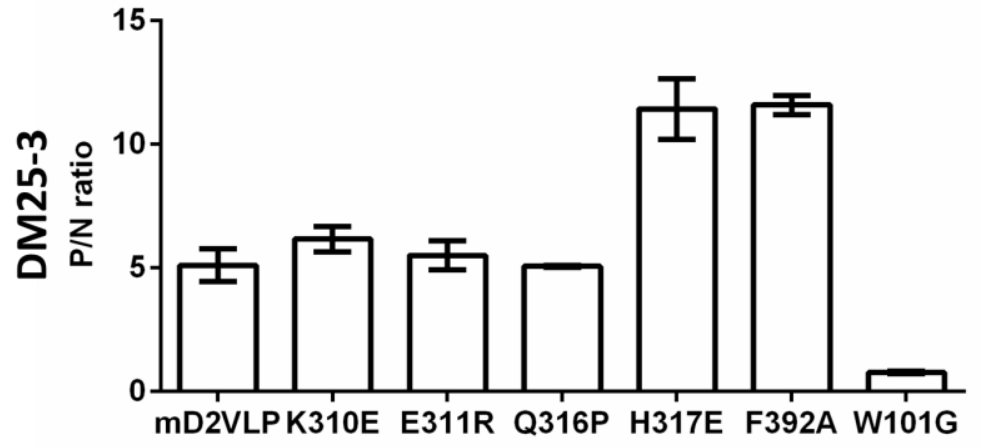

\begin{tabular}{cccc} 
Relative binding $(\%)$ & W101G & K310E & E311R \\
\hline 4G2 & $9 \pm 0.7$ & $109.6 \pm 5.2$ & $99.4 \pm 7.5$ \\
4A1B-9 & $46.3 \pm 8.2$ & $84.3 \pm 12.2$ & $83.9 \pm 4.7$ \\
6B3B-3 & $12.1 \pm 0.1$ & $111.9 \pm 5.7$ & $82.8 \pm 8.2$ \\
6B6C-1 & $40.6 \pm 3$ & $127.1 \pm 19.8$ & $101 \pm 18.9$ \\
DM25-3 & $5.6 \pm 0.3$ & $128.6 \pm 6.6$ & $98.4 \pm 5.9$ \\
T5-1 & $93.4 \pm 11.2$ & $12.5 \pm 2.1$ & $30.9 \pm 2.7$ \\
DM8-6 & $119.5 \pm 1.8$ & $89.2 \pm 2$ & $41.7 \pm 0.5$ \\
\hline
\end{tabular}

1185

1186

1187 Figure S12. Identification of the neutralizing epitopes of MAbs DM25-3 and DM8-6. (A) Bar

1188 graph shows the decrease in MAb reactivity in a binding-ELISA for mD2VLPs with

1189 substitutions at the designated residues. Various mD2VLP mutants were expressed in COS-1

1190 cells by electroporation with plasmid DNAs as indicated. Tissue culture media were

1191 harvested 3 days after transfection and clarified for antigen-capture ELISA. Substitutions at 
1192 N103K, G104Q, K307E, E314R, T315H, P364R and W391G produced plasmids which did

1193 not secrete measurable VLP antigens into the tissue culture medium. Therefore, only mutant

1194 plasmids indicated secreted VLP antigens and were used for epitope mapping by binding

1195 ELISA. (Top) Substitutions of E311E led to a significantly reduced binding activity of

1196 neutralizing MAb DM8-6, compared to mD2VLP. (Bottom) Substitutions of W101G led to a

1197 complete loss of binding by MAb DM25-3. Data shown are a representative experiment of

1198 three independent experiments. (B) To ensure that the antigenic structures were intact, mutant

1199 VLPs including W101G, K310E and E311R were further confirmed by using a panel of

1200 DENV-2 MAbs, including group-cross-reactive antibodies (4G2, 4A1B-9, 6B3B-3, 6B6C-1,

1201 DM25-3) recognizing all four major pathogenic flavivirus serocomplexes; complex cross-

1202 reactive antibodies (T5-1) recognizing all four DENV complex viruses, and type-specific

1203 antibody (DM8-6) recognizing DENV-2 only. The relative binding of MAbs was determined

1204 by dividing the $\mathrm{OD}_{450}$ of $\mathrm{mD} 2$ VLP mutants by that of $\mathrm{mD} 2 \mathrm{VLP}$. The data are presented as means

$1205 \pm$ SEM from three independent experiments with two replicates. The two-tailed Mann-Whitney $U$ test

1206 was used to test statistical 

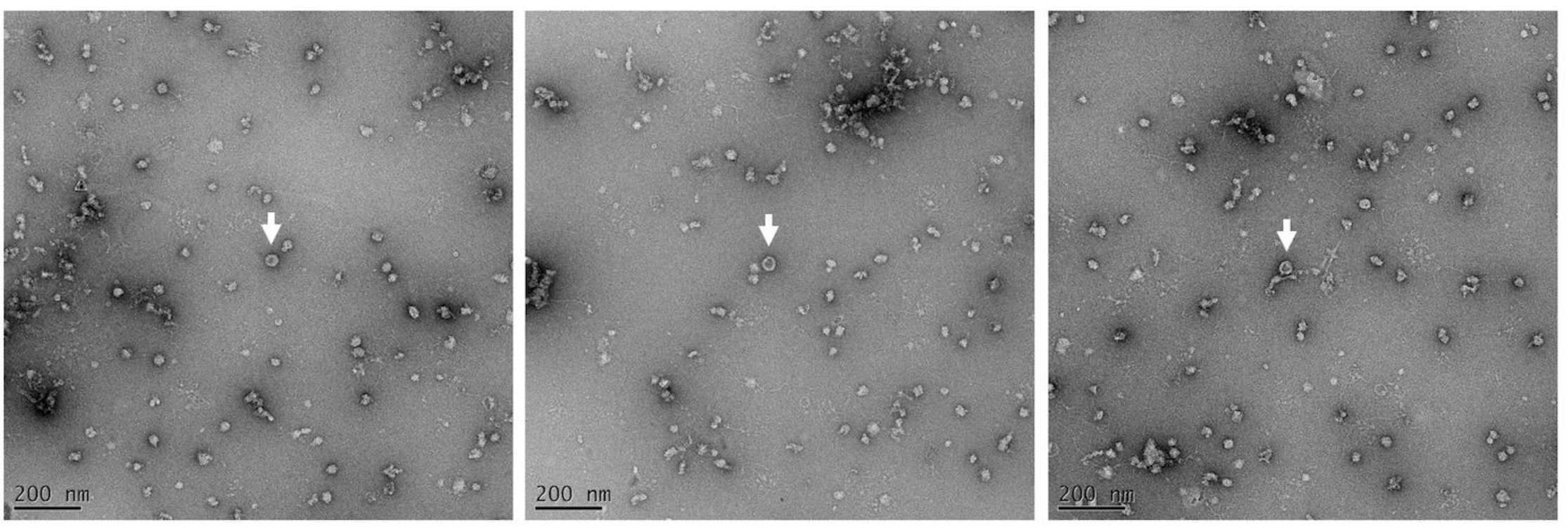

1207

1208 Figure S13. Transmission electron microscope (TEM) examination of negatively stained W101G-mutant mD2VLP. Purified W101G-mutant mD2VLP were adsorbed onto a glow-discharged carbon coated grid (EMS CF-200-Cu) for 1min, and inspected by TEM. The images were taken by a JEM1400 electron 


\begin{tabular}{|c|c|c|c|}
\hline \multirow[b]{2}{*}{$\mathrm{VH} 1$ : 1} & \multicolumn{3}{|l|}{ CDR2 } \\
\hline & GEPGTSLKISCKTSGYTFTEYTMYWVKQSHGKSLEWIGGINPNNGGTT & TYNQKFKGKATLT & 60 \\
\hline & CDR3 & & \\
\hline $\mathrm{VH} 2: 61$ & VNKSSSIAYMEVRNLTSEDSAVYYCVRYGGYYVFDYWGQGTTLTVSS & 107 & \\
\hline & CDR1 & CDR2 & \\
\hline VL1 : 1 & TLWKTQSPASLAVSLGQRATISYRASKSVSTSGYSYMHWNQQKPGQPF & PRLLIYLVSNLES & 60 \\
\hline & & & \\
\hline /L2 : 61 & GVPARFSGSGSGTDFTLN & 07 & \\
\hline
\end{tabular}

1217 Figure S14. Amino acid sequences of variable regions of IgH and IgL of antibody DM25-3. The amino acids in the CDR regions are 1218 highlighted in red. 

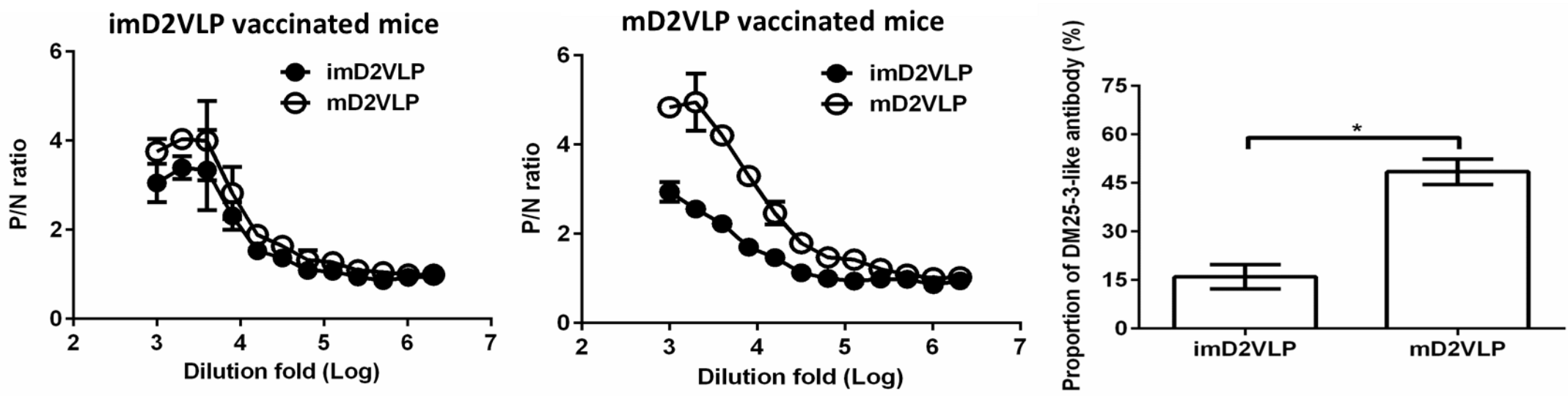

Figure S15. Binding ELISA was performed to test the reactivity of sera from mice immunized with two doses of imD2VLP or mD2VLP against equal amounts of imD2VLP and mD2VLP antigens. The difference in binding activity was converted to a bar chart at 1:1000-fold dilutions of mice sera. Proportions of DM 25-3-like antibodies from two different D2VLP immunization groups were calculated based on the formula $100 \mathrm{x}\left(\mathrm{OD}_{450} \mathrm{mD} 2 \mathrm{VLP}-\mathrm{OD}_{450} \mathrm{imD} 2 \mathrm{VLP}\right) / \mathrm{OD}_{450} \mathrm{mD} 2 \mathrm{VLP}$. The data are presented as means \pm SEM from three independent experiments with two replicates. The two-tailed Mann-Whitney $U$ test was used to test statistical significance. ${ }^{*}, P<0.05$. 


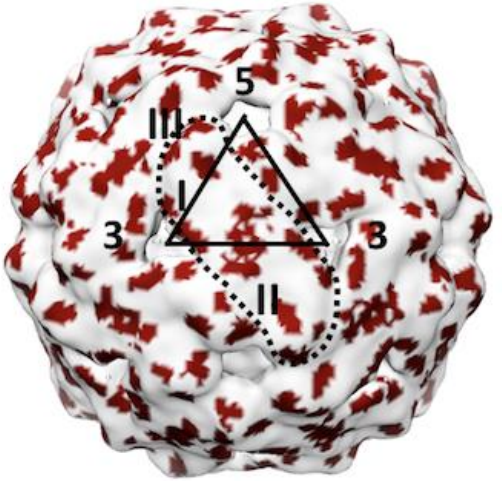

1227

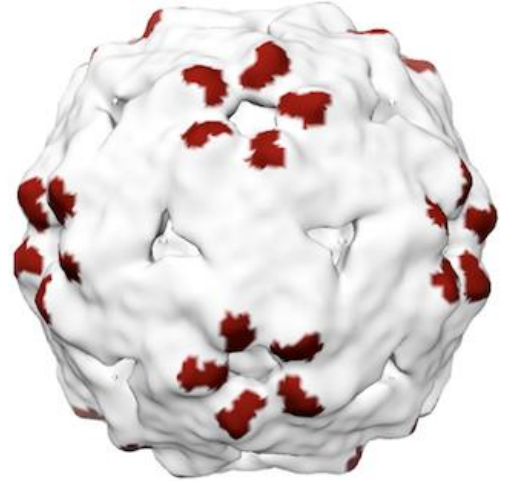

1A1D-2

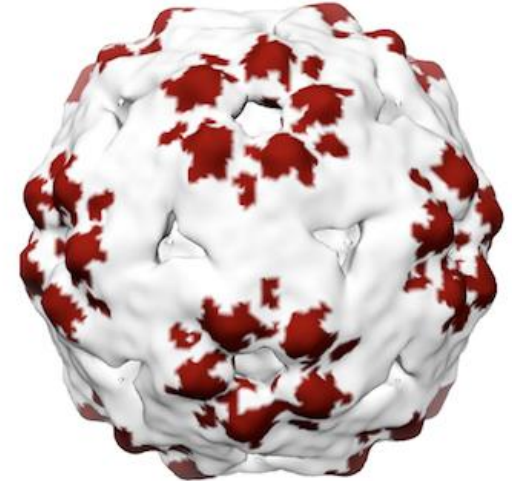

2D22

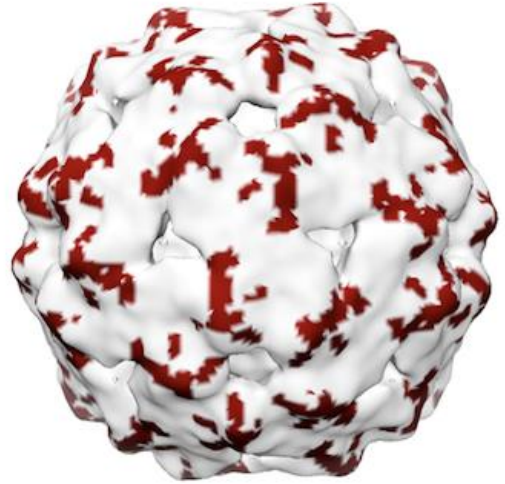

EDE

Figure S16. The "neutralization-sensitive hotspot" on mD2VLPs. (Left) The highly exposed residues $(\Delta \%$ SASA $\geq 0.2)$ which were colored by dark red color are shown on the surface rendered VLP. The E dimer is outlined by a black dash line, the symmetry and the domains are labeled. The E epitopes interacting with MAb 1A1D-2 (center left), 2D22 (center right) and EDE (right) are colored in dark red on the surface showing that they were highly exposed on the mD2VLP surface and were highly overlapping with areas of highly exposed residues in mD2VLP. 